\title{
Cosmic Rays and Ultra High Energy Cosmic Rays (UHECR), GZK (Greisen-Zatsepin-Kuzmin) cutoff, Deflection angle of high energy cosmic rays in the propagation through the galactic and extragalactic magnetic fields, Auger Surface Detector and the Monitoring Data, Measurement of the Lifetime of Muons and Pions and The Variant Mass for a Particle in a Gravitational Field and the Binary Stars: The Physics and emission of the gravitational energy
}

Giovanni Alcocer

Independent Research, Guayaquil, Ecuador.Email: giov_alc_science@hotmail.com

Master in Physics with specialization in Astrophysics and Medical Physics, Professor of Physics, Advanced Mathematics and Science in general, author of the recognized and renowned articles: The Fundaments of the Mass Gravitation, Electromagnetism and Atom. Pipo Zona: Great sportsman, champion and scorer of many national and international championships, a sportman of the high elite who always competes with love and respect for his teammates and the Football ball without which this beautiful sport could not be practiced thanks to the Supreme Being.

DOI: http://doi.org/10.46382/MJBAS.2021.5201

Copyright: (C2021 Giovanni Alcocer. This is an open access article distributed under the terms of the Creative Commons Attribution License, which permits unrestricted use, distribution, and reproduction in any medium, provided the original author and source are credited.

The Surface Detector of the Pierre Auger Observatory consists of 1600 water Cherenkov tanks sampling ground particles of air showers produced by energetic cosmic rays. Every water tank is equipped with three photomultipliers (PMTs). Every 7 minutes monitoring data are recorded from each surface detector to ensure that they are performing as intended and also to understand the detector behaviour. The monitoring data set is used to study the evolution of parameters of the PMTs as a function of both time and temperature. The main objective of the monitoring data is to monitor the stability of the PMTs. In case there is abnormal behaviour detected, an alarm entry is created in the database. The classification of the alarms into different categories, corresponding to the observed patterns of the PMT variables and the decision of the alarm level are subject of this work. It is also researched the photomultipliers parameters and the alarm creation with examples of it: the Free Disk and High PMT gain change Alarms using $\mathrm{c}++$, root and mysql. For other hand, it is researched about the measurement of the lifetime of muons and pions theoretically, experimentally and with the application of Statistics for the uncertainties of the results. The results have high accuracy and in accordance with the values of the literature.

In addition, it is to analyze theoretically the deflection angle of the high energy cosmic rays in the propagation through the galactic and extragalactic magnetic fields. It is used the magnetic force formula and the Larmor radius, trigonometry, and the tables for the values of the galactic and extragalactic magnetic field and the distances to the galactic and extragalactic sources. It is possible to observe from this analysis that the deflection angles are very small for the considered cases. Thus, UHECR arrival directions should point back to their sources in the sky [32].

Besides, it is researched about the GZK (Greisen-Zatsepin-Kuzmin) cutoff. A sharp cutoff at approximately several times $1019 \mathrm{eV}$ in the energy spectrum of the cosmic rays is expected due to the energy degradation of the cosmic rays through their interaction with photons of the microwave background radiation. It is known as the GZK (Greisen-Zatsepin-Kuzmin) cutoff. A nucleon with an initial energy above Eo=1020 eV lose about $1 / 5$ of Eo (initial energy) in each interaction. Thus, a nucleon of initial energy above $1020 \mathrm{eV}$ will reduce its energy to $6^{*} 1019 \mathrm{eV}$ (GZK cutoff energy) after traveling a distance of approximately $50 \mathrm{Mpc}$ [32].

For other hand, Albert Einstein wrote in a research article: "Does the inertia of a body depend on its energy content?" (Ist die Trägheit eines Körpers von seimen Energienhalt abhängig?) [33]: "If a body emits energy E in the form of radiation, its mass decreases by E/c2". Thus, Maxwell's theory shows that electromagnetic waves are radiated whenever charges accelerate as for example for the electrón. Then, this electromagnetic radiation (photons) produces a decrease in the mass of the electron which is given by the formula of the Variant Mass for an Accelerated Charged Particle which was demonstrated by me at this research [34] and at the research of the Variant Mass of the Electron at the Atom [36]. This is true for any type of radiation emitted: electromagnetic or gravitational energy which produce a decrease in the mass of the body.

Therefore, other objective of this article is to demonstrate the discovered formula which describes exactly the variant mass for a particle which emits gravitational energy [33]. An example of the effect of this Gravitational energy emission is the light deflection for the light passing close the Sun (gravitational redshift frequency) and the Perihelion Precession of Mercury. Thus, the results of the mass formula are of great relevance for Gravitational Interactions. The results are in accordance with the classic result for the emission of the total gravitational energy (bond total energy) for a particle orbiting a large Planet or Sun and for a Binary Star. It is in agreement with the experiment result and with the Theory of General Relativity [33].

Keywords: Cosmic Rays, Ultra High Energy Cosmic Rays, Deflection angle, High energy cosmic rays, Galactic and extragalactic magnetic fields, GZK (Greisen-Zatsepin-Kuzmin) cutoff, Auger Surface Detector, Monitoring Data, Measurement of lifetime of muons and pions, Variant Mass, Gravitational Energy, Total Bond Energy, Binary Stars. 


\section{Cosmic Rays and Ultra High Energy Cosmic Rays}

Julius Elster and Hans Geitel noticed an unknown ionizing source in the air in their research of the atmospheric electricity. Independently, Charles Wilson suspected the existence of an ionization agent that could penetrate a thick layer of earth by using the ionization chamber. They reasoned that the ionization could be caused by an unknown radiation of large penetration other than X-ray and radioactivity, because even when the ionization chamber was shielded, they observed ionization. Wilson called it "cosmic radiation" because he supposed that the radiation might come from extraterrestrial sources. In 1911, Victor Hess discovered the cosmic rays by using a balloon-borne pressurized ionization chamber [1]. Firstly, a slight diminution of ionization was observed by him while the balloon went up and after he detected a fast increase of ionization radiation that persisted up to an altitude of $5 \mathrm{~km}$. Later, Werner Kolhörster investigated this radiation of extremely high penetrability with more refined techniques that confirmed the ionization until an altitude of $9 \mathrm{~km}$. The absorption coefficient was much smaller than the absorption coefficient of gamma rays. So, the conclusion was that the ionization agent came from a high level and it was confirmed and called Cosmic Radiation (Höhenstrahlung). Besides, D.V. Skobeltzin observed tracks of charged particles by using a cloud chamber in his cosmic ray investigations. Also, Walter Bothe and Kolhörster identified charged particles by using Geiger-Müller counters.

The most fantastic suggestion of the origin of cosmic rays was made by Robert Millikan at his interpretation of the energy spectrum of primary cosmic rays derived from the latitude effect observed by means of the balloon flight experiments. Millikan thought that the cosmic rays could be due to the spontaneous destruction of nuclei, such as carbon, nitrogen, and oxygen. But it was not convincing, and the hypothesis of mass annihilation is only of historical interest. For other hand, Oscar Klein took into account the option that the matter and the antimatter forming galaxies and antigalaxies could annihilate to release an enormous amount of energy. But this process cannot be responsible for particles with energies above $1 \mathrm{Tev}$. Therefore, this idea can be a possible mechanism of energy release but only just that. In addition, Fred Hoyle assumed the possibility of the ejection of very heavy nuclei in supernova outbursts. However, also it cannot explain the presence of the energetic particles. An alternative theory was the Galactic origin of cosmic rays proposed by Fermi who proposed the statistical acceleration produced from collisions of charged particles with interstellar clouds. In 1938, Pierre Auger noticed time coincidence in two surface detectors separated located many meters from each other and he observed showers with energies of around $10^{15} \mathrm{eV}$. So, Auger had discovered the "air shower", showers of secondary particles caused by the collision of primary high energy particles with molecules of the atmosphere.

In 1960, cosmic microwaves background (CMB) was discovered by Arno Penzias and Robert Wilson. Afterward, Kenneth Greisen, Vadem Kuzmin and Georgi Zatsepin concluded that cosmic rays of high energies would interact with the microwave background radiation. In this process, the energy of those high energy cosmic rays would be reduced and so particles traveling a long distance could not have energies more a threshold energy of a few times $10^{19} \mathrm{eV}$. It is known as the GZK (Greisen- Zatsepin-Kuzmin) cutoff. But in 1991, the Fly's Eye cosmic ray team in the U.S detected a cosmic ray with an energy of $10^{20} \mathrm{eV}$. In 1994, also an event with energy of around $10^{20}$ 
$\mathrm{eV}$ was reported in Japan in the AGASA experiment [2]. In order to analyze those high energy cosmic rays with more statistics the Pierre Auger Observatory in Malague, Argentina was designed in 1995. The origin and the evolution of the Universe could be understood by tracing high energy cosmic rays to their unknown source.

\subsection{Air Showers}

Due to all of these investigations, we know now that the cosmic rays are composed of the following components: a) the hadronic component (nucleon component), b) muon and neutrino component and c) soft component (electromagnetic component: Beta and gamma ray). Also, we can distinguish between primary cosmic rays and secondary cosmic rays. Primary cosmic rays are incident at the top of the atmosphere and secondary cosmic rays are produced by the primary rays in the propagation through the atmosphere. Primary cosmic rays consist mainly of protons (80-90\%), alpha particles (10-15\%), helium nuclei and nuclei of heavier element with atomic number more or equal than 3 up to uranium (1\%), and electrons or positrons (2\%) [3]. Primary particles that are capable to penetrate the earth magnetic field collide with the nuclei of the earth atmosphere and thus, secondary particles are produced with enough energy to induce further reactions. Therefore, the secondary particles originate from the collision of the primary particle against the nuclei in the atmospheric air. The primary interaction is hadronic and occurs at an altitude of approximately $20 \mathrm{~km}$ above the sea level. In each interaction, part of the energy of the primary particle is lost in the production of secondary hadrons (principally pions) [4] [6]. The secondary particles can decay or interact again with an atom of our atmosphere and so on, inducing a cascade process of particles called Extensive Air Shower (EAS), which only stops if the energy of the secondary particles is lower than the threshold for further nuclear process (approximately $1 \mathrm{GeV}$ ). The reaction products of an EAS can be detected with giant detectors on the Earth surface. All particles produced in an EAS are called cosmic radiation.
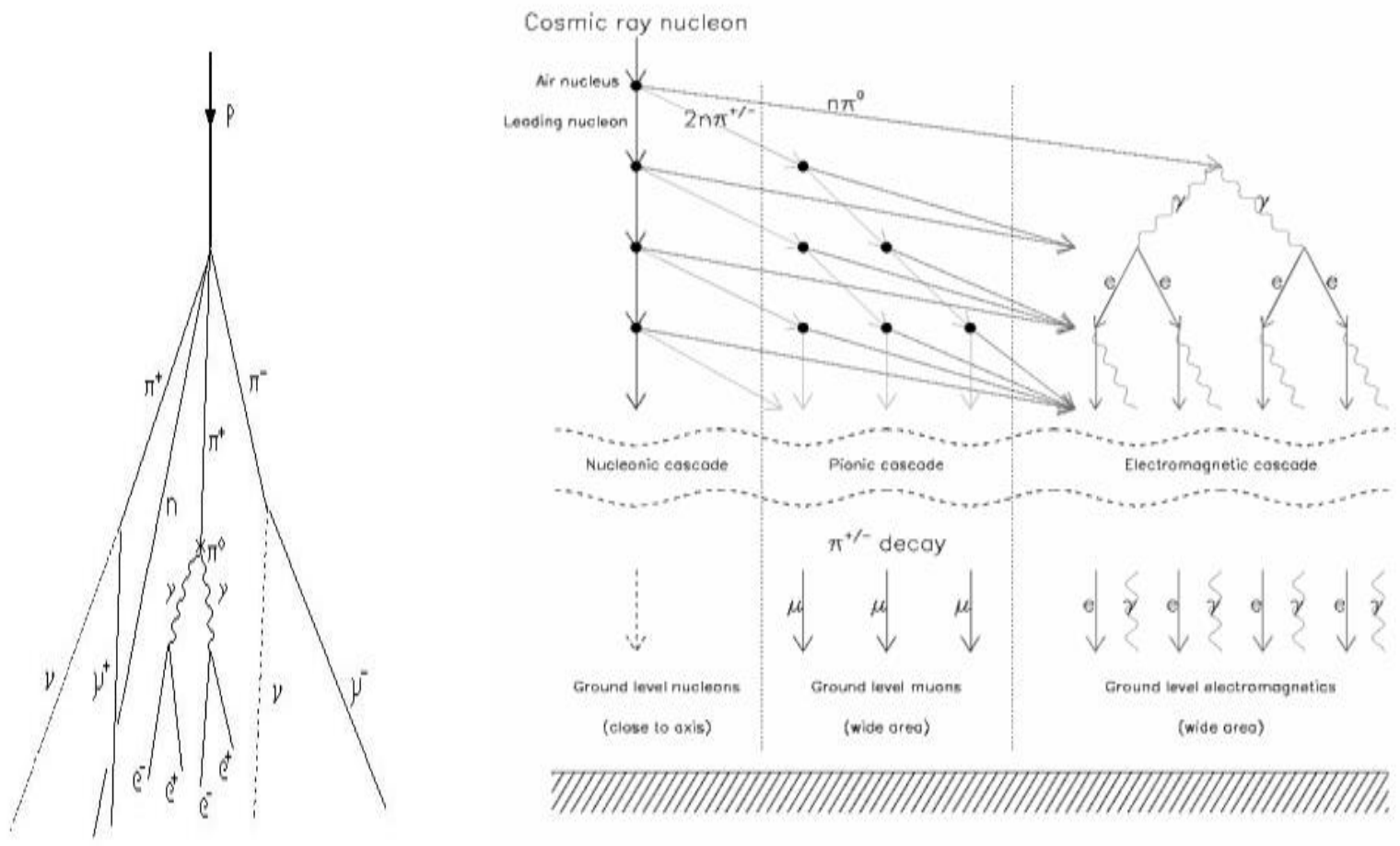

Fig.1. Schematic showing the developing of the cosmic radiation (EAS) in the atmosphere [13] 
The hadronic process can be described by the reaction:

$N+N_{\text {air }} \rightarrow N^{\prime}+N^{\prime}$ air $+\pi^{0}\left(\pi^{ \pm}, K^{0}, K^{ \pm}, n, p, \ldots\right)$

Where, $\mathrm{N}$ is the primary or secondary particle of the cosmic radiation and $\mathrm{N}_{\text {air }}$ is a nucleus of the atmosphere. The pions constitute most of the secondary particles of the hadronic component (around of $65 \%$ ) [5]. Some of the particles produced in the hadronic interactions are neutral pions, which mostly decay into two photons: $\pi^{0} \rightarrow \gamma+\gamma$

These photons initiate the electromagnetic cascade formed of photons, electrons and positrons. At each hadronic interaction, about $30 \%$ of the hadronic component is transferred to the electromagnetic component [5]. Because the hadrons do many interactions, the most part of the initial energy is given to the electromagnetic component of the shower.

In the electromagnetic component numerous photons, electrons and positrons are produced by Bremsstrahlung and pair production processes. Thus, photons, electrons and positrons are the most numerous particles in the evolution of the shower (around $99 \%$ and carrying about $85 \%$ of the shower energy) [5]. Electrons, positrons and photons are absorbed rapidly (soft component) by interaction processes of them with the atmosphere and it causes that the electromagnetic component vanishes [4]. Other particles generated in the development of the air shower, are the charged mesons. The dominant ways of decay of them are:

$$
\begin{aligned}
& \pi^{+} \rightarrow \mu^{+}+v_{\mu} \\
& \pi^{-} \rightarrow \mu^{-}+\overline{v_{\mu}} \\
& K^{+} \rightarrow \mu^{+}+v_{\mu} \\
& K^{-} \rightarrow \mu^{-}+\overline{v_{\mu}} \\
& K^{+} \rightarrow \pi^{+}+\pi^{0} \\
& K^{-} \rightarrow \pi^{-}+\pi^{0}
\end{aligned}
$$

At the sea level, the secondary cosmic radiation is classified essentially into the soft component (electromagnetic component: beta, gamma rays) and muons components. The muons form the muonic component and they decay with a lifetime of $2,2 * 10^{-6}$ s. Due to their highly relativistic velocity, many of them reach the earth surface. The number of muons in the evolution of the shower is around 1\% [5]. Muons are sufficiently energetic (hard component), rarely interact and only decay under weak interactions.

It causes that they can reach the sea level and penetrate deep underground, but it is necessary to consider the relativistic effects. Therefore, about $80 \%$ of the charged particles at sea level are muons [4]. Pions and kaons are also produced in the hadronic cascade and local production by protons, neutrons mainly and they also can reach the sea level. In the cascade process, muons and pions decays generate neutrinos and they constitute the neutrino component. Finally, the hadrons that survive the interactions contribute to the hadronic component [5]. 


\subsection{Energy Spectrum of the Cosmic Rays}

The energy spectrum of primary cosmic rays can be described as a increase power law: $\frac{d F}{d E} \propto E^{-\delta}$ where $\mathrm{F}$ is the flux of the cosmic rays, $\mathrm{E}$ is the energy of them and $\delta$ is the spectral index. The figure 2 shows a plot of the flux of cosmic rays versus their energy. The spectrum can be divided into three regions well-known with the names of knee (two first regions) and ankle (third region). The first knee appears around $3 * 10^{15} \mathrm{eV}$ where the spectral index is between 2.7 and 3.0 [5]. The second knee is located around $10^{17}$ and $10^{18} \mathrm{eV}$ where the spectral index is between 3.0 and 3.3. The ankle is in the region of $3 * 10^{18} \mathrm{eV}$. Above this energy the spectrum is more concave with a spectral index of 2.7. For these energies the flux of the particles is less than 1 particle per $\mathrm{km}^{2}$ per year.

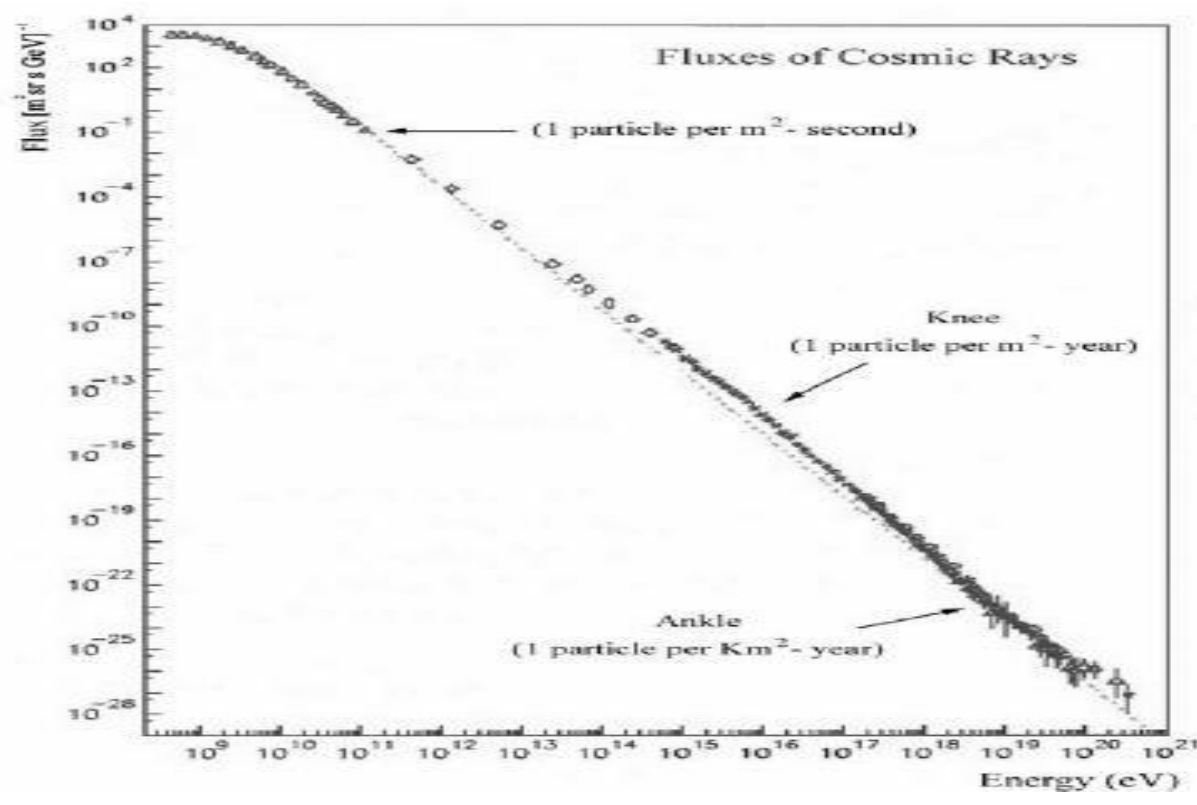

Fig.2. Fluxes of Cosmic Rays [14]

The origin of the cosmic rays for the first two regions of the energy spectrum is in our galaxy and their energies can be explained by the acceleration mechanism of Fermi. For the third region, there are many open questions like origin of cosmic rays, primary composition and acceleration mechanisms.

\subsection{Ultra-High Energy Cosmic Rays (UHECR)}

In 1930, Pierre Auger and his colleagues observed coincidences between two or more detectors separated by a distance of more than $300 \mathrm{~m}$. They associated those coincidences with showers of cosmic rays (EAS) triggered by the arrival of a single cosmic ray of high energy at the top of the atmosphere [7]. In 1991, Hungye Dai was analyzing the data from the Fly's Eye experiment in the Utah sky. The faint flash of ultraviolet light produced in the sky when the cosmic ray particles hit the upper atmosphere was monitored and a very high energy event of $3 * 10^{20} \mathrm{eV}$ was reported. Other experiments like the Akeno Giant Air Shower (AGASA) in Japan (1993-2004) announced other events with energies above $10^{20} \mathrm{eV}$ [8]. In 1995, Cronin and Watson began to 
impel the PAO, an international effort that involves 15 countries: Argentina, Australia, Bolivia, Brazil, Czech Republic, France, Germany, Italy, Poland, Mexico, Slovenia, Spain, United Kingdom, USA and Vietnam [9]. They had the vision of two observatories, one in the Northern hemisphere and another one in the Southern hemisphere in order to cover the entire sky.

\section{The GZK cutoff}

A sharp cutoff at approximately several times $10^{19} \mathrm{eV}$ in the energy spectrum of cosmic rays is expected due to the energy degradation of the cosmic rays through their interaction with photons of the microwave background radiation. It is known as the GZK (Greisen-Zatsepin-Kuzmin) cutoff [32]. Primary protons in this range of energies collide with photons of the microwave background radiation and enter in the region of resonances (unstable barions) via the equations:

$\gamma+p \rightarrow \Delta^{+}$

$\Delta^{+} \rightarrow p+\pi^{0}$

$\Delta^{+} \rightarrow n+\pi^{+}$

Therefore, it is obtained two reactions for the unstable barions:

$\gamma+p \rightarrow p+\pi^{0}$

$\gamma+p \rightarrow n+\pi^{+}$

Appling four momentum conservation for the barion reaction of proton:

$\gamma+p \rightarrow p+\pi^{0}$ : for a proton with mass M, momentum $\mathrm{p}$, Energy E, pion of mass $\mathrm{m}$, microwave photon $\gamma$ with momentum q and energy qc (in order to simplify the initial formulations, we use natural units, so $c=1$ ):

$(E+q)^{2}-(p+q)^{2}=(M+m)^{2} \quad(E+q)^{2}-(p+q)^{2} c^{2}=(M+m)^{2} c^{4}$

$M^{2}+2 q(E-|p| \cos \theta)=M^{2}+m^{2}+2 M m \quad\left(E^{2}-p^{2} c^{2}=M^{2} c^{4}\right)$

For a head-on collision $\theta=180^{\circ}$ (most favourable case for the production of a reaction) and so $(1-\cos \theta)=2$. Due to the fact that the proton is extreme relativistic $\left(\mathrm{E}>>\mathrm{Mc}^{2}\right)$, we have $\mathrm{E} \approx \mathrm{p}$ and so the resulting equation for the threshold energy is:

$4 q E=m(m+2 M)$

$E=m \frac{\left(M+\frac{m}{2}\right)}{2 q}$

Returning to the initial units:

$E=m c^{2} \frac{\left(M+\frac{m}{2}\right) c^{2}}{2 q c} \quad($ Threshold energy $)$

Where: $\quad \mathrm{m}=139,57 \mathrm{MeV} / \mathrm{c}^{2}$ (pion mass), $\mathrm{M}=938,27 \mathrm{MeV} / \mathrm{c}^{2}$ (proton mass), and $\mathrm{qc}$ is the energy of the photon. 
In order to determine this energy, we calculate the peak energy of the photons of the cosmic microwaves background (CMB) [32]. Appling Wien's law and the Einstein equation for the energy of photons:

$\lambda_{\text {peak }}=\frac{2.9 * 10^{6}}{\mathrm{~T}}[\mathrm{~nm}] \quad($ Wien's law $)$

$\mathrm{E}_{\text {peak }}=\frac{\mathrm{hc}}{\lambda_{\text {peak }}} \quad$ (Einstein equation: $\left.\mathrm{E}=\mathrm{hf}=\mathrm{hc} / \lambda\right)$

Replacing $\mathrm{T}=2,7 \mathrm{~K}$ (temperature of the microwave background) and $\mathrm{hc}=1240[\mathrm{eV}-\mathrm{nm}]$, the typical CMB photon energy is around qc=1.16 meV. Finally replacing this value in the equation of the threshold energy E, it is obtained:

$E=m c^{2} \frac{\left(M+\frac{m}{2}\right) c^{2}}{2 q c}$

$\mathrm{E} \approx 6,1 * 10^{19} \mathrm{eV}$ (Threshold energy for the proton)

It is possible to do the calculations with the barion reaction of neutron obtaining exactly the same threshold energy [32]:

$\gamma+p \rightarrow n+\pi^{+}$

Appling four momentum conservation for the reaction:

$\gamma+p \rightarrow n+\pi^{+}:$for a proton with mass M, momentum p, Energy E, pion of mass $\mathrm{m}$, neutron of mass $\mathrm{m}^{\prime}$, microwave photon $\gamma$ with momentum q and energy qc (in order to simplify the initial formulations we use natural units, so $c=1)$ :

$(E+q)^{2}-(p+q)^{2}=\left(m^{\prime}+m\right)^{2} \quad(E+q)^{2}-(p+q)^{2} c^{2}=\left(m^{\prime}+m\right)^{2} c^{4}$

$M^{2}+2 q(E-|p| \cos \theta)=m^{\prime 2}+m^{2}+2 m^{\prime} m \quad\left(E^{2}-p^{2} c^{2}=M^{2} c^{4}\right)$

For a head-on collision $\theta=180^{\circ}$ (most favourable case for the production of a reaction) and so $(1-\cos \theta)=2$. Due to the fact that the proton is extreme relativistic $\left(\mathrm{E}>>\mathrm{Mc}^{2}\right)$, we have $\mathrm{E} \approx \mathrm{p}$ and so the resulting equation for the threshold energy is:

$4 q E=m^{\prime 2}+m^{2}+2 m^{\prime} m-M^{2}$

$E=\frac{m^{\prime 2}+m^{2}+2 m^{\prime} m-M^{2}}{4 q}$

Returning to the initial units:

$E=\frac{\left(m^{\prime 2}+m^{2}+2 m^{\prime} m-M^{2}\right) c^{4}}{4 q c} \quad($ Threshold energy $)$

Where: $\quad \mathrm{m}=139,57 \mathrm{MeV} / \mathrm{c}^{2}$ (pion mass), $\mathrm{M}=938,27 \mathrm{MeV} / \mathrm{c}^{2}$ (proton mass), $\mathrm{m}^{\prime}=939,57 \mathrm{MeV} / \mathrm{c}^{2}$ (neutron mass) and qc is the energy of the photon [32]. In order to determine this energy, we calculate the peak energy of the photons of the cosmic microwaves background (CMB) [32]. Appling Wien's law and the Einstein equation for the 
energy of photons:

$\lambda_{\text {peak }}=\frac{2.9 * 10^{6}}{\mathrm{~T}}[\mathrm{~nm}] \quad($ Wien's law $)$

$\mathrm{E}_{\text {peak }}=\frac{\mathrm{hc}}{\lambda_{\text {peak }}} \quad($ Einstein equation: $\mathrm{E}=\mathrm{hf}=\mathrm{hc} / \lambda)$

Reemplacing $\mathrm{T}=2,7 \mathrm{~K}$ (temperature of the microwave background) and $\mathrm{hc}=1240[\mathrm{eV}-\mathrm{nm}]$, the typical CMB photon energy is around qc $=1.16 \mathrm{meV}$. Finally reemplacing this value in the equation of the threshold energy E, it is obtained:

$E=\frac{\left(m^{\prime 2}+m^{2}+2 m^{\prime} m-M^{2}\right) c^{4}}{4 q c}$

$\mathrm{E} \approx 6.1 * 10^{19} \mathrm{eV}$ (Threshold energy for the neutron, which is the same threshold energy of the proton).

Therefore, a nucleon with initial energy above $10^{20} \mathrm{eV}$ lose about $1 / 5$ of $\mathrm{E}_{\mathrm{O}}$ (initial energy) in each interaction and the mean free path for collision is given by the formula:

$\lambda=\frac{1}{\rho \sigma}$

Where, $\sigma$ is the cross section near the threshold energy $\left(6.1 * 10^{19} \mathrm{eV}\right)$ for the interaction of high energy protons with photons of the microwave background radiation. The values of $\sigma$ and $\rho$ where $\rho$ is the microwave photon density are:

$\sigma=2 * 10^{-28} \mathrm{~cm}^{2}$

$\rho=400 \mathrm{~cm}^{-3}$

Replacing those values, it is obtained $\lambda \approx 9.5 \mathrm{Mpc}$ (where $1 \mathrm{Mpc}=1.31 * 10^{24} \mathrm{~cm}$ ). Appling the simple exponential model for the energy evolution of the shower [32]:

$E=E_{o} e^{-\epsilon l}$

Where:

1 is the traveling distance of the nucleon (mean free path for the collision)

$\epsilon$ is a constant to be determined by replacing:

$\mathrm{E}_{\mathrm{o}}=10^{20} \mathrm{eV}$

$\mathrm{E}=6.1 * 10^{19} \mathrm{eV}$ (cutoff energy),

$1=\lambda=9.5 \mathrm{Mpc}$

Thus, the value of the constant $\epsilon$ is:

$\epsilon=0.05 \mathrm{Mpc}^{-1}$ 
And the resulting equation is:

$\mathrm{E}=\mathrm{E}_{\mathrm{o}} \mathrm{e}^{-0.051}(\mathrm{l}$ in Mpc $)$

This means that the energy of the nucleon is reduced by a factor of $e^{-0.051}$. Thus, a nucleon of initial energy above $10^{20} \mathrm{eV}$ will have its energy reduced to $6.1 * 10^{19} \mathrm{eV}$ (GZK cutoff energy) after traveling a distance of approximately $10 \mathrm{Mpc}$ given by last formula [32].

For larger initial energy, the cross section $\sigma$ increases and leads to a similar maximal distance of less than $100 \mathrm{Mpc}$ [32].

It is possible to conclude:

- A nucleon at Earth, which traveled a distance more than $100 \mathrm{Mpc}$, will have always an energy less than $10^{20} \mathrm{eV}$ independent of the initial energy.

- Particles with an observed energies above $10^{20} \mathrm{eV}$ are limited to distances of approximately less than $100 \mathrm{Mpc}$ from the Earth [32].

Nevertheless the AGASA experiment showed a significant number of events with energies beyond the GZK cutoff and for this reason a giant air shower array, Pierre Auger Observatory is built to study these very rare high energy cosmic rays [2]. The energy spectrum of the AGASA experiment is shown in the next figure where it is possible to observe some events with energies more than $10^{20} \mathrm{eV}[2]$

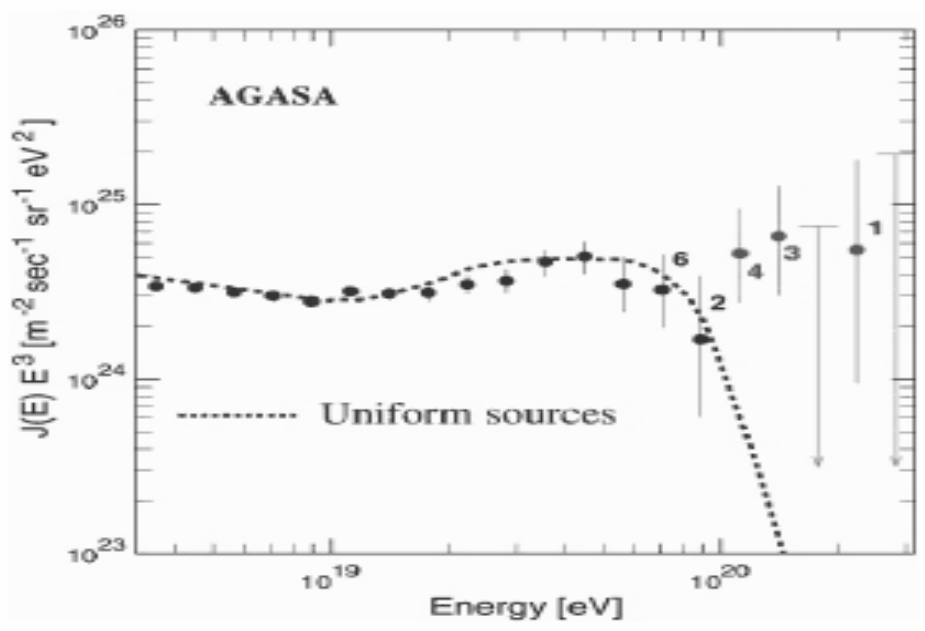

Fig.3. Energy spectrum from AGASA experiment (dots). The plot with the bars is the expected spectrum assuming the cosmological uniform distribution of sources [2]

3. Deflection angle of high energy cosmic rays in the propagation through the galactic and extragalactic magnetic fields.

In order to calculate the deflection of a charged cosmic ray in extragalactic and galactic magnetic fields, the derivation of the most simple formula is given below [32]. 
The particles enter in the magnetic field with their velocities being perpendicular to the magnetic field lines.

Thus, the angle between $\mathrm{v}$ and $\mathrm{B}$ is $90^{\circ}$ and it is obtained: $\mathrm{F}=\mathrm{q}|\mathrm{vxB}|=\mathrm{qvBsen} \theta$ where $\theta=90^{\circ}$ and $\mathrm{F}=\mathrm{qvB}$. Therefore, the force due to the magnetic field $\mathrm{B}$ will act on the charged particle $\mathrm{q}$ via the equation: $\mathrm{F}=\mathrm{qvB}$

Where, $\mathrm{B}$ is the magnetic field directed away from us. The magnetic force acting on the particle is perpendicular to its movement or the velocity direction (right hand rule) [32]. Thus, the particle is forced to move on a circular path with radius $r_{B}$ as it is shown in the next figure:

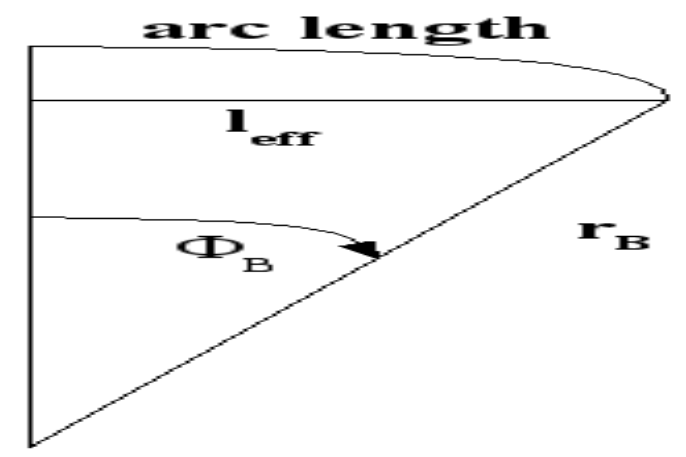

Fig.4. Deflection of the charged particle in the magnetic field

This force is also equal to the centrifugal force:

$F=\frac{m v^{2}}{r_{B}} \quad$ Where, $m$ is the mass of the particle and $r_{B}$ is the radius of the circular trajectory.

Equaling both equations the resulting equation for the radius $r_{B}$ is:

$\mathrm{qvB}=\frac{\mathrm{mv}^{2}}{\mathrm{r}_{\mathrm{B}}} \quad \mathrm{r}_{\mathrm{B}}=\frac{\mathrm{mv}}{\mathrm{qB}} \quad \mathrm{p}=\mathrm{mv} \quad \mathrm{r}_{\mathrm{B}}=\frac{p c}{\mathrm{qcB}} \quad \mathrm{E}=\mathrm{pc} \quad \mathrm{E}^{2}=\mathrm{p}^{2} \mathrm{c}^{2}+\mathrm{m}^{2} \mathrm{c}^{4} \quad \mathrm{E}>>\mathrm{mc}^{2} \quad \mathrm{E} \approx \mathrm{pc}$ (high energy cosmic rays) $r_{B}(m)=\frac{E(J o u l e)}{q(\text { Coulombs }) c\left(\frac{m}{s}\right) B(\text { Tesla })} \quad$ (units in the International System SI)

In order to bring the equation to astrophysic units, it is necessary to use the conversions:

$1 \mathrm{eV}=1.6 * 10^{-19} \mathrm{~J}, 1 \mathrm{~T}($ Tesla $)=10^{4}$ Gauss, $\mathrm{B} n \mathrm{ng}=\left(\mathrm{B} / 10^{-9}\right)(\mathrm{B}$ in Gauss $), \mathrm{q}=1.6^{*} 10^{-19} \mathrm{C} \quad \mathrm{E} 20=\mathrm{E} / 10^{20}(\mathrm{E}$ in eV), 1 $\mathrm{Mpc}=1.31 * 10^{24} \mathrm{~cm}, \mathrm{Z}$ is added in the electric charge.

Thus, the Larmor radius of a cosmic nucleus traveling in a magnetic field is:

$$
\mathrm{r}_{\mathrm{B}}=\frac{\mathrm{E}(\mathrm{J})}{\mathrm{Z}\left(1.6 * 10^{-19}\right) \mathrm{cB}} * \frac{1.6 * 10^{-19}}{1.6 * 10^{-19}} * \frac{10^{4}}{10^{4}} * \frac{10^{20}}{10^{20}} * \frac{10^{-9}}{10^{-9}}=\frac{\mathrm{E}_{20}(\mathrm{eV}) * 10^{4} * 10^{20}}{\mathrm{Z}\left(3 * 10^{8}\right) \mathrm{B}_{\mathrm{ng}}(\mathrm{G}) * 10^{-9}} * \frac{100 \mathrm{~cm}}{1 \mathrm{~m}} * \frac{1 \mathrm{Mpc}}{1.31 * 10^{24} \mathrm{~cm}}
$$

Where, $\mathrm{E}(\mathrm{eV})=\mathrm{E}(\mathrm{J}) / 1.6^{*} 10^{-19}$ and $\mathrm{B}(\mathrm{G})=\mathrm{B}($ Tesla $) * 10^{4}$ and $\mathrm{E}_{20}=\mathrm{E}(\mathrm{eV}) / 10^{20}$ and $\mathrm{B} n \mathrm{~g}=\left(\mathrm{B}(\mathrm{G}) / 10^{-9}\right)$

$\mathrm{r}_{\mathrm{B}} \approx \frac{100 * \mathrm{E}_{20}}{\mathrm{ZB}_{\mathrm{ng}}} \mathrm{Mpc} \quad$ where $\mathrm{B}_{\mathrm{ng}}=\left(\mathrm{B}(\mathrm{G}) / 10^{-9}\right)$ and $\mathrm{E}_{20}=\mathrm{E}(\mathrm{eV}) / 10^{20}$ and $\mathrm{Z}$ is the atomic number of the particle.

For the angle deflection of the particle in the magnetic field (see figure 4), the equation is: $\sin \Phi_{B}=\frac{l_{\text {eff }}}{r_{B}}$ 
Where: $1_{\text {eff }}$ is the path lenght (in units of $\mathrm{Mpc}$ ).

Therefore, it is possible to replace the Larmor radius and the next equation is obtained:

$\sin \Phi_{\mathrm{B}}=\frac{\mathrm{l}_{\mathrm{eff}} \mathrm{ZB}_{\mathrm{ng}}}{100 E_{20}}$

The next equation is obtained by considering the approximation for small angles:

$\Phi_{\mathrm{B}}<<1: \quad \Phi_{\mathrm{B}} \approx \frac{\mathrm{l}_{\mathrm{eff}} \mathrm{ZB} \mathrm{ng}}{100 E_{20}}$

Then, it is important to consider the thresholds values of the path length $l_{\text {eff }}$ and the galactic and the extragalactic magnetic fields in order to have the maximum and the minimum angles [32]. They are given in the next table:

Table 1. Threshold values for the magnetic fields and distances to the sources [10], [11], [12]

\begin{tabular}{|c|c|}
\hline Values of the Galactic Magnetic Field & $10^{-8} \mathrm{G}<\mathrm{B}<10^{-6} \mathrm{G}$ \\
\hline Values of the Extragalactic Magnetic Field & $10^{-12} \mathrm{G}<\mathrm{B}<10^{-9} \mathrm{G}$ \\
\hline Distances to Galactic Sources & $\mathrm{l}_{\mathrm{eff}}<30 \mathrm{kpc}$ \\
\hline Distances to Extragalactic Sources & $\mathrm{l}_{\mathrm{eff}}>30 \mathrm{Mpc}$ \\
\hline
\end{tabular}

We consider $\mathrm{E}=1 * 10^{20} \mathrm{eV}$ and two cases of particles: a proton $(\mathrm{Z}=1)$ and Iron $(\mathrm{Z}=13)$, product of stellar evolution) where $1_{\text {eff }}$ is in $\mathrm{Mpc}, \mathrm{B}_{\mathrm{ng}}=\left(\mathrm{B}(\mathrm{G}) / 10^{-9}\right), \mathrm{E}_{20}=\mathrm{E}(\mathrm{eV}) / 10^{20}$.

We use the threshold of the path length for extragalactic magnetic field leff $=30 \mathrm{Mpc}, \mathrm{E}=1 * 10^{20} \mathrm{eV}$ and the both limits of the extragalactic magnetic field: $10^{-12} \mathrm{G}<\mathrm{B}<10^{-9} \mathrm{G}$. Thus, we can obtain the next table 2:

Table 2. Deflection angles, of cosmic rays which travel through the extragalactic magnetic field

\begin{tabular}{|c|c|c|}
\hline $\mathbf{B}$ & $\mathbf{Z}$ & $\mathbf{\Phi}_{\mathbf{B}}\left({ }^{\circ}\right)$ \\
\hline $1 * 10^{-12} \mathrm{G}$ & 1 & $3 * 10^{-4}$ \\
\hline $1 * 10^{-12} \mathrm{G}$ & 13 & $3.9 * 10^{-3}$ \\
\hline $1 * 10^{-9} \mathrm{G}$ & 1 & 0.3 \\
\hline $1 * 10^{-9} \mathrm{G}$ & 13 & 3.9 \\
\hline
\end{tabular}

For the galactic magnetic field, it is used the threshold of the path length $\mathrm{l}_{\mathrm{eff}}=0.030 \mathrm{Mpc}, \mathrm{E}=1 * 10^{20} \mathrm{eV}$ and the both limits of the galactic magnetic field: $10^{-8} \mathrm{G}<\mathrm{B}<10^{-6} \mathrm{G}$ [32]. Therefore, it is obtained the next table 3:

Table 3. Deflection angles of cosmic rays which travel through the galactic magnetic field.

It is possible to consider the special case of the galactic center [32]

\begin{tabular}{|c|c|c|}
\hline $\mathbf{B}$ & $\mathbf{Z}$ & $\left.\boldsymbol{\Phi}_{\mathbf{B}} \mathbf{(}^{\circ}\right)$ \\
\hline $1 * 10^{-8} \mathrm{G}$ & 1 & $3 * 10^{-3}$ \\
\hline $1 * 10^{-8} \mathrm{G}$ & 13 & $3.9 * 10^{-2}$ \\
\hline $1 * 10^{-6} \mathrm{G}$ & 1 & 0.3 \\
\hline $1 * 10^{-6} \mathrm{G}$ & 13 & 3.9 \\
\hline
\end{tabular}


leff $=8,5 \mathrm{kpc}=0,0085 \mathrm{Mpc}$, with $\mathrm{B}=1 * 10^{-7} \mathrm{G}, \mathrm{E}=1 * 10^{19} \mathrm{eV}$ and $\mathrm{Z}=13$ (iron) and the deflection angle is $1.1^{\circ}$.

For $\mathrm{Z}=1$ (proton), it is possible to observe from the tables that the deflection angles are very small for the considered cases. Thus, UHECR arrival directions should point back to their sources in the sky [32]. Therefore, an experiment able to provide data with high quality and statistical significance in the upper-end of the cosmic ray spectrum, good angular resolution and the uniform exposure over the whole sky is necessary. The Pierre Auger Observatory was built with these objectives.

\section{The Pierre Auger Observatory (PAO)}

The main objectives of the Pierre Auger Observatory (PAO) are to understand the nature, origin and the propagation of UHECR. The PAO is designed for complete-sky coverage with an aperture of at least 7350 $\mathrm{km}^{2} \mathrm{sr}$ in each hemisphere in order to detect events with energies above $10^{19} \mathrm{eV}$. The southern observatory campus is located in Malague, Argentina [9]. The PAO is measuring energy, arrival direction and mass composition of cosmic rays with very high statistical precision with two sub-systems: a Surface Detector (SD) and a Fluorescence Detector (FD). The array of surface detectors measures the lateral and temporal distribution of shower particles at ground level. The Surface Detector of the PAO is composed of 1600 water Cherenkov detectors arranged in a hexagonal grid with $1.5 \mathrm{~km}$ spacing between detectors. They cover a total area of 3000 $\mathrm{km}^{2}$. The layout is shown in Figure 5 .

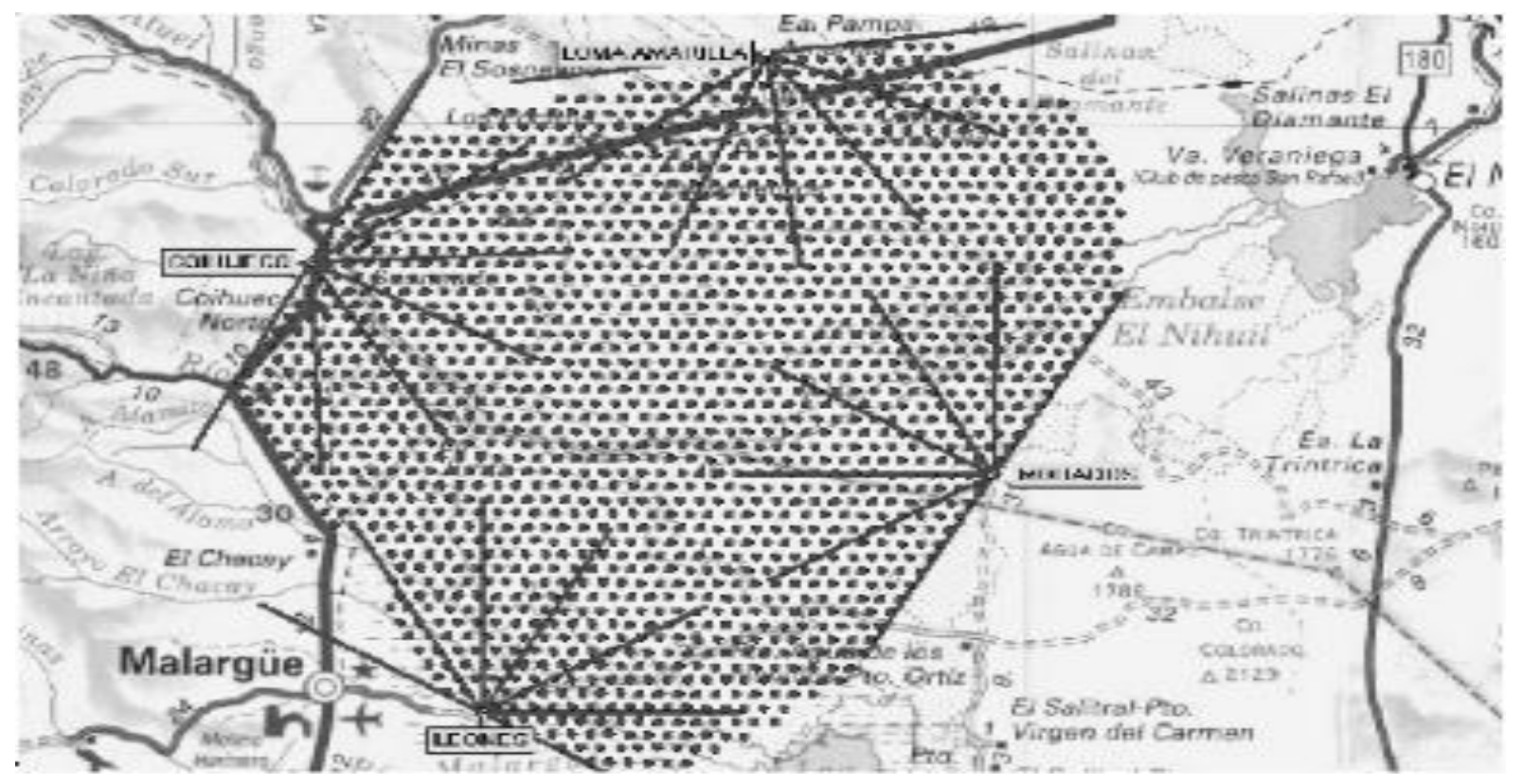

Fig.5. Layout of the Pierre Auger Observatory (dots are the SD tanks) [16]

A water Cherenkov detector is shown in Figure 6. Each detector station is a cylindrical tank with a surface of $10 \mathrm{~m}^{2}$ and a height of $1.2 \mathrm{~m}$, filled with 1200 litres of purified water [2]. Three photomultipliers collect the Cherenkov light emitted by charged particles in the EAS as they cross the tank. These stations operate on battery-backed solar power and communicate with a central station (central data acquisition system: CDAS) by using radio links. Event timing is provided through Global Positioning System (GPS) receivers. 


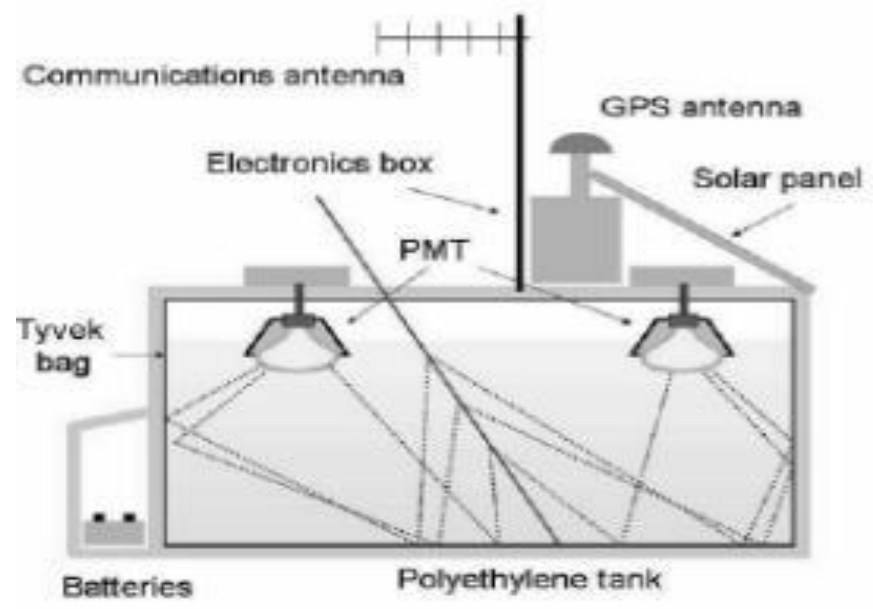

Fig.6. Schematic view of a water Cherenkov tank [15]

The Fluorescence Detector of the PAO is composed of 4 stations located at: Loma Amarilla, Coihueco, Morados, Leones (see figure of the layout of the Pierre Auger Observatory), each station having 6 telescopes. The 24 fluorescence telescopes are overlooking the array. They measure the longitudinal development of the shower in the atmosphere. In the mirror focus of each telescope 440 phototubes are installed as a camera. The 4 stations measure the emission from atmospheric nitrogen which is excited by the charged particles of the shower as they traverse the atmosphere and emit fluorescence light.

The PAO has been designed to work in a hybrid detection mode. The next figure shows the design fo the hybrid detection of an air shower:

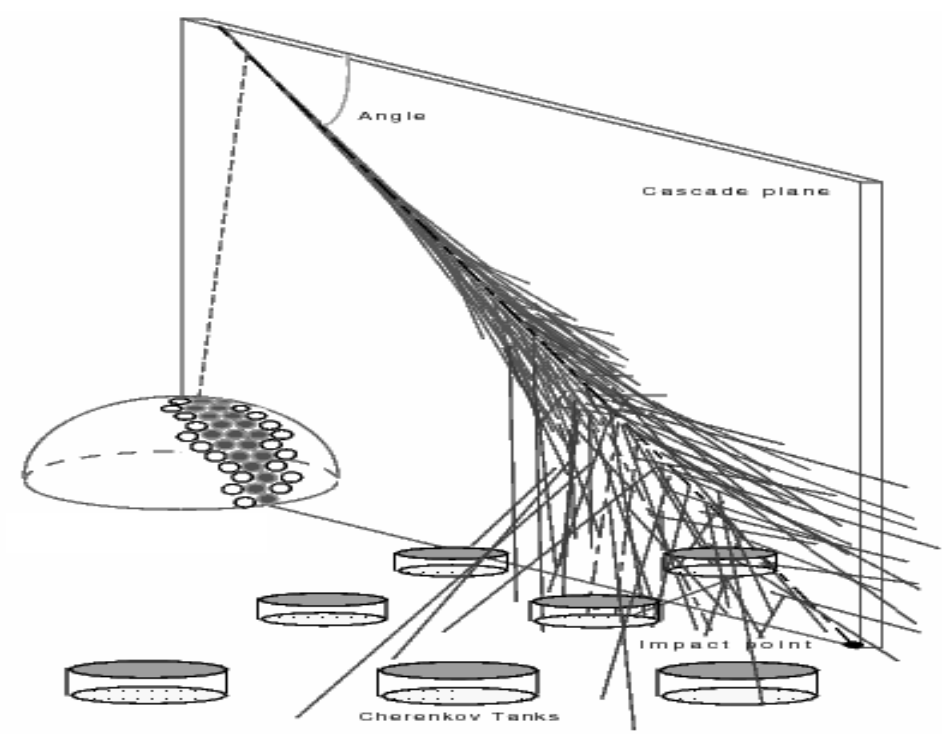

Fig.7. Schematic view of an air shower measured by both SD and FD. Black circles indicate the fired phototubes and the large cylinders are the water Cherenkov [17]

This hybrid technique will allow to measure the energy and the arrival direction with high precision as well as very good separation of protons and heavy nucleus. The energy resolution for the hybrid technique is around $10 \%$ at $10^{20} \mathrm{eV}$ and the angular resolution is approximately $0.5^{\circ}$ [2]. If the detection is performed only with the Surface 
array the energy resolution is between $10 \%$ and $20 \%$ and the angular resolution varies from $1^{\circ}$ and $2^{\circ}$.

\subsection{Photomultiplier Signal}

The secondary cosmic rays are observed by the emission of the Cherenkov light, as they transverse the water of a Surface Detector tank. The Cherenkov light in one detector station is detected by 3 photomultiplier tubes (PMTs). The signal for an event of a cosmic ray air shower is mainly generated by electrons and muons in the shower. Other types of particles are insignificant. The energy of the electrons is lower than that of the muons and they cause smaller signals. The signal of a single muon recorded by a PMT in one SD tank is shown in the next figure. The typical rise time of a muon signal is $10 \mathrm{~ns}$ with a decay time of around $70 \mathrm{~ns}$. The figure shows the single vertical muon signal recorded by a PMT of the Surface station as it is observed with an oscilloscope:

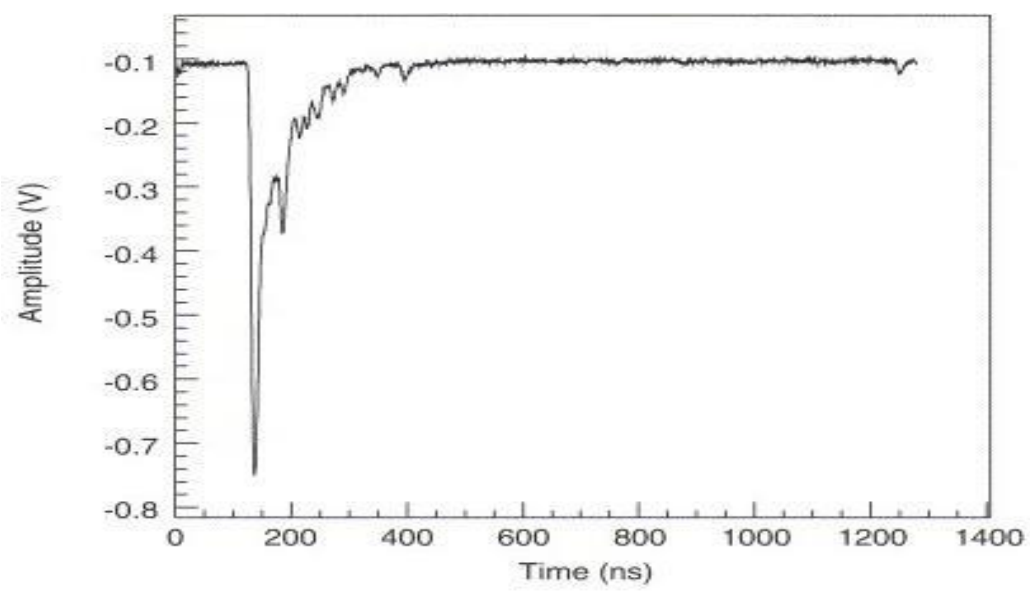

Fig.8. Single muon signal recorded by a PMT of a water cherenkov tank [18]

From each PMT of one station, the signal from the anode and from the last dynode are sent to the local station acquisition system (The Front-End board with 3 low gain channels (anode) and 3 high-gain channels (last dynode)). The signal of the dynode is inverted and amplified to an amount of 32 times to match the Dynamic range.
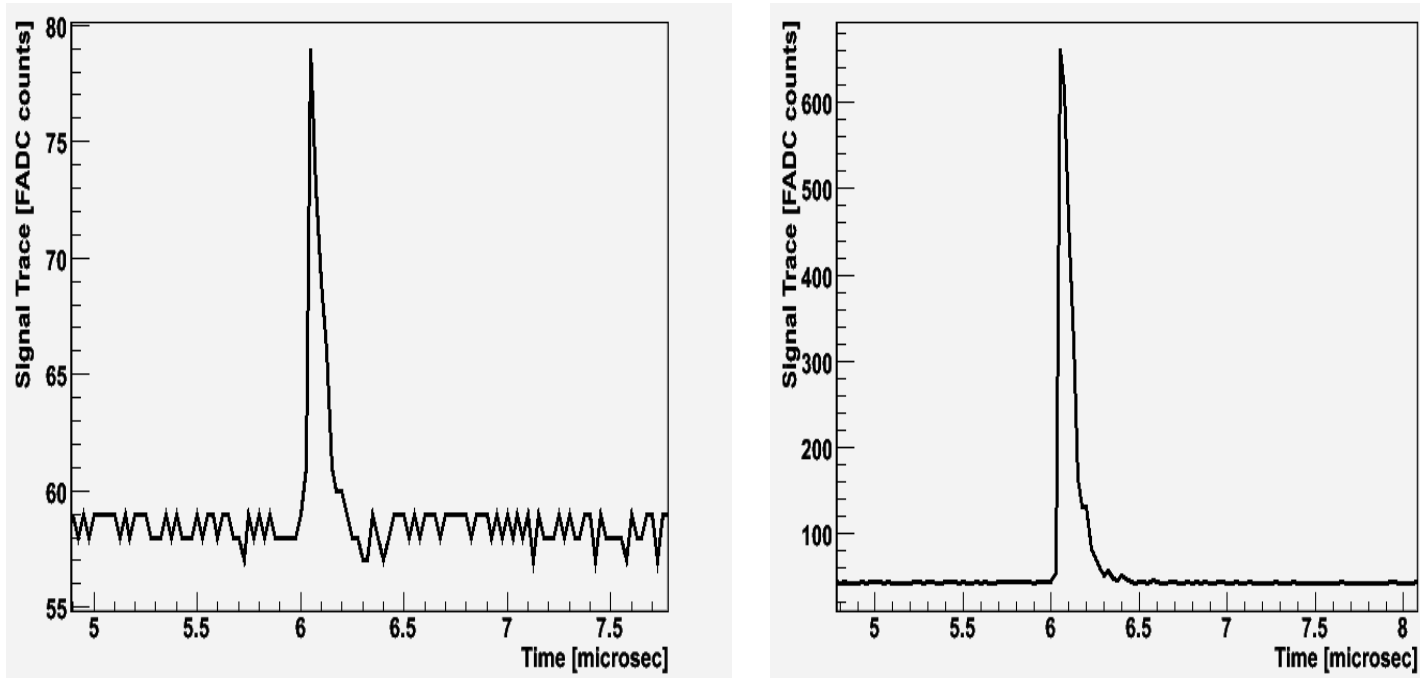

Fig.9. ADC traces recorded (anode: left, dynode: right) from the FADC of one of the PMT of the Surface detector (LsId=201, PMT3) 
A single vertical muon generates a peak signal of approximately 30 photoelectrons per PMT in the Interval of 25 ns. Nevertheless, for a shower of $10^{21} \mathrm{eV}$, the signal has around 20000 photoelectrons per PMT at a distance of $500 \mathrm{~m}$ from the shower axis [19]. It is necessary to have enough Dynamic range for the signal in order to cover with very good precision the particle flux near (around 100 particles us $^{-1}$ ) and far away (approximately 1 particle $\mathrm{us}^{-1}$ ) the shower core. Thus, two channels: one with low gain and one with high gain, are used for each PMT. In order to have good calibration for both channels, a 5 bit overlap for both channels has been chosen. Afterward, the ADC outputs are forwarded to the trigger system.

\subsection{The Surface Detector Calibration}

Calibration of the Surface Detector (SD) is based on the average signal deposited in the tank by a single high energy muon traversing a tank vertically. This signal is called the vertical equivalent muon (1 VEM). The aim of the calibration procedure is to convert the ADC signal of the PMTs into VEM units and to obtain a uniform trigger for the Surface detector. In order to have reference values for the calibration, firstly measurements of the photocurrent and the charge deposited in a reference tank by a single vertical and central muon were done. Atmospheric muons passing through the detector give an excellent method to measure the value of 1 VEM, because they produce a peak in a pulse height histogram ( ${ }^{\text {peak }}$ VEM).

$\mathrm{I}^{\text {peak }} \mathrm{VEM}$ is defined as the peak photocurrent produced by a vertical through going muon and corresponds about 50 ADC counts for each PMT in the low gain channel [23]. This value was measured by using an external muon telescope from external triggers and by comparison with the measurements from the internal station trigger [31]. The muon telescope uses two scintillators, placed on the top and bottom of one detector tank situated at the central campus. The pulse height histogram for one PMT is shown in the next figure where the peak at around 50 ADC counts is due to single muon crossing the tank.

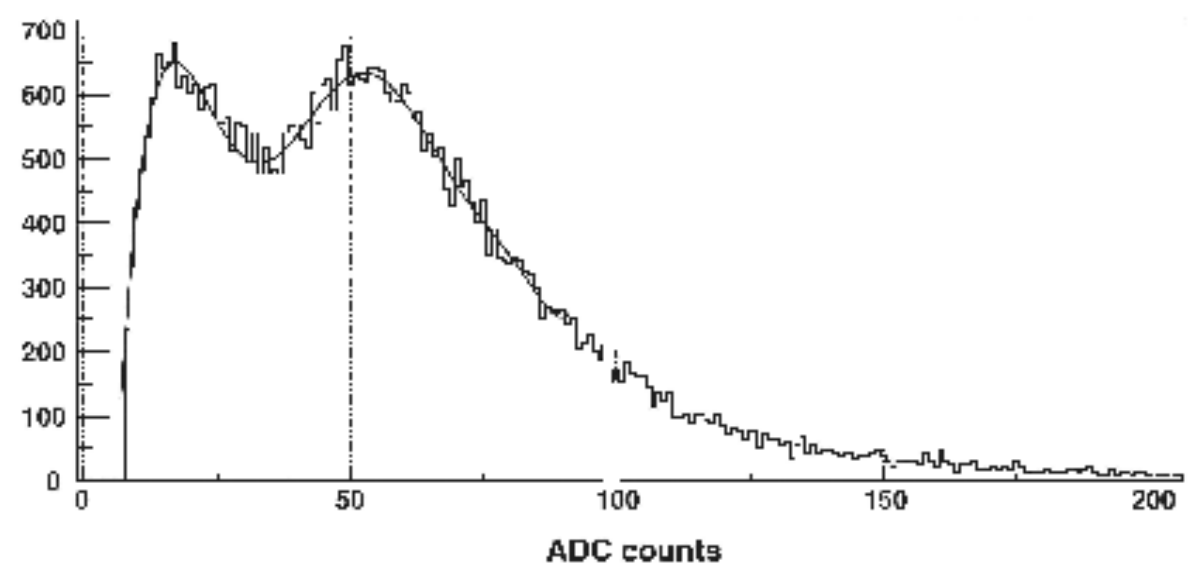

Fig.10. Pulse height histogram for a single PMT of a reference tank [24]

This peak $\mathrm{I}^{\text {peak }}$ VEM is related to the average charge deposited by a vertical and central through going muon, because the charge is the integrated signal of the current. Thus, the atmospheric muons also produce a peak $\left(\mathrm{Q}^{\text {peak }} \mathrm{VEM}\right)$ in a charge histogram. Therefore, $\mathrm{I}^{\text {peak }} \mathrm{VEM}$ is the peak in a pulse height histogram and $\mathrm{Q}^{\text {peak }} \mathrm{VEM}$ is the peak in a charge histogram. The peak $\mathrm{Q}^{\text {peak }} \mathrm{VEM}$ is at around 1.09 VEM summed over all 3 PMTs and 
$(1.03 \pm 0.02)$ VEM for each PMT. This was measured in a special measurement with a muon telescope in a reference tank [20]. The next figure shows the peak $\mathrm{Q}^{\text {peak }} \mathrm{VEM}$ in a charge distribution for one PMT measured by using a muon telescope.

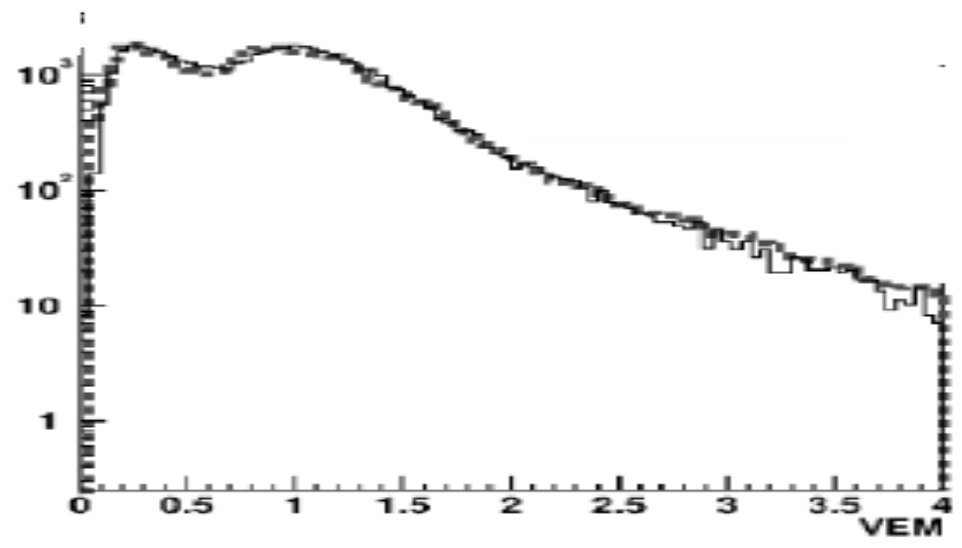

Fig.11. Charge histogram for one PMT of one reference tank in units of VEM (the peak is approximately is at 1.03 VEM) [22]

The calibration of the Surface Detector is based on a procedure in three steps:

1) Set up the gains of each PMT at $\mathrm{I}^{\text {peak }} \mathrm{VEM}=50 \mathrm{ADC}$ counts, by adjusting the high voltage of each PMT in order to have the trigger rates above a common threshold for the three PMTs, using as reference unit for the threshold trigger the $\mathrm{I}^{\text {peak }}$ VEM.

2) The development of the gains is monitored (local calibration) in order to adjust the level triggers and compensates drifts of the step 1 .

3) The $I^{\text {peak }}$ VEM is stabilized by the step 2, the trigger is enabled and a set of charge and pulse height histograms are produced and sent to the central data acquisition system. The histograms have about 15000 entries per minute. Finally, we use the conversion factor from $\mathrm{Q}^{\text {peak }}$ VEM to VEM (1.03 \pm 0.02$)$ in order to have the conversion from the average of all simples to VEM units [23], [31].

In the next figure, it is possible to observe examples of the charge integrated signal (charge histogram) and the amplitude signal (pulse height histogram) from a SD triggered by a 3-fold coincidence between all 3 PMTs with all PMTs summed. The dashed lines in the histogram are provided using an external muon telescope to select only the VEM.
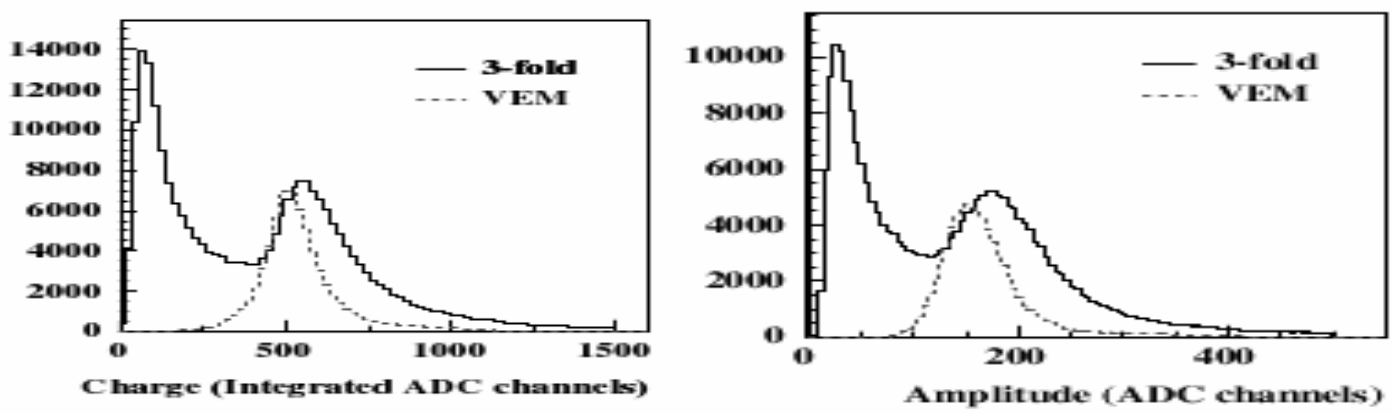

Fig.12. Charge histogram (left side) and Pulse height histogram (right side) from a SD station, triggered by a 
3-fold coincidence between all 3 PMTs with the signal from all 3 PMTs summed (dashed histogram is measured using an external muon telescope to have only the VEM signal) [20]

The histograms are understood as the convolution of three diverse classes of next incoming particles:

(1) Muons which enter through the top and exit through the bottom (VEM) (second peak).

(2) Muons which enter through the top or bottom and exit through the side (first peak).

(3) Muons which enter through the side and exit through the side (firs peak).

The first peak is due the contribution of the second and third classes together with the spreading of the signal due low energy particles. The second peak is due vertical through going atmospheric muons.

The histogram of the number of photoelectrons from a vertical muon for a single PMT is also shown in the next figure:

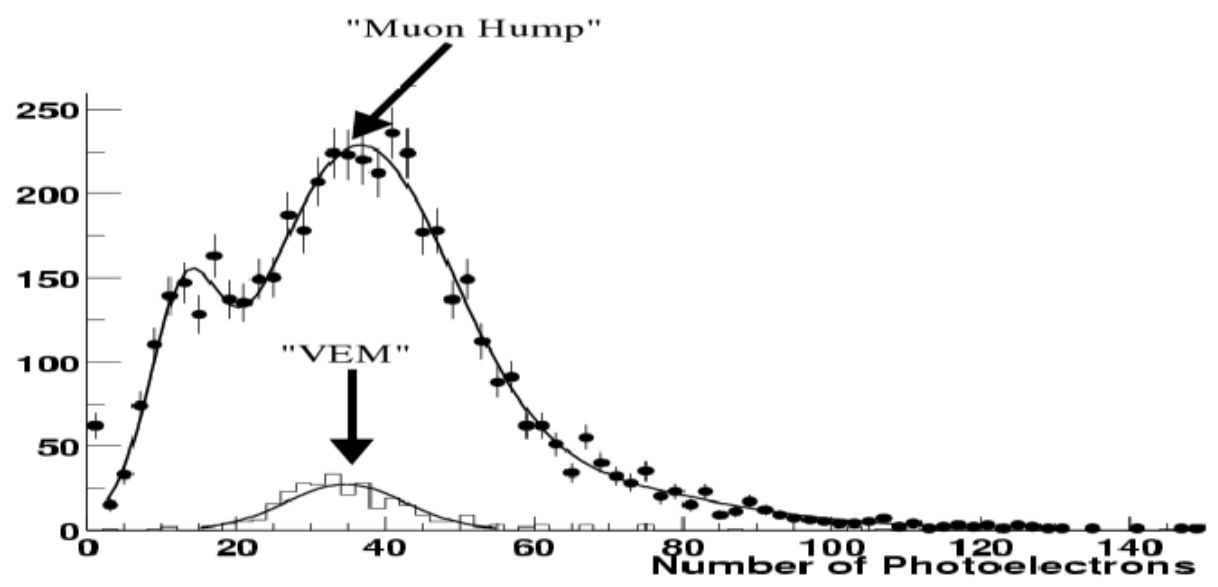

Fig.13. Charge distribution (number of photoelectrons) for a single PMT resulting from vertical muons crossing through the tank (solid line histogram) using muon trigger scintillators placed on the top and bottom of the reference tank, and from "internal trigger", simply requiring three fold coincidence of three PMTs in a tank [21] It is possible to observe in the figure that the peak (1 VEM) corresponds to approximately 30 photoelectrons.

\subsection{The Trigger System}

The trigger system of the Surface station is hierarchic being composed of more trigger levels with local triggers in levels 1 (T1) and 2 (T2). The level 3 (T3) is formed at the Central Data Acquisition System (CDAS) based on the spatial and temporary correlation of the trigger level T2. All the data that satisfy T3 trigger will be stored. Additional levels of triggers are used offline to select physical events (T4) and accurate events (T5).

$\mathrm{I}^{\text {peak }}$ VEM is the common reference unit for the threshold of the triggers. The first trigger level $\mathrm{T} 1$ is evaluated by the PLD (programmable logic device) board and it is composed of two different trigger modes:

- Three-fold coincidence of a $1.75 \mathrm{I}^{\text {est }}$ VEM threshold on each PMTs and $2.5 \mathrm{I}^{\mathrm{est}} \mathrm{VEM}$ for one PMT. The $\mathrm{I}^{\text {est }}$ VEM is the estimated current for a Vertical Equivalent Muon. This trigger is used to detect fast signals 
(muonic component).

- Time over Threshold (ToT): 13 bins within a window of 120 bins above a threshold of $0.2 \mathrm{I}^{\mathrm{est}}$ VEM in coincidence for two PMTs. It has a low rate of $1.6 \mathrm{~Hz}$ which corresponds for the expected rate of double muons for an Auger Surface Detector. This trigger is used to detect small and spread signals [25].

A signal may satisfy both conditions and the priority is given by the ToT. The triggers $\mathrm{T} 2$ are evaluated by the local station system (local electronic is controlled by a CPU board that has a Power PC at $40 \mathrm{MHz}$ ) and select from the T1 signals those probable ones to have come from EAS. All the ToT triggers are promoted to T2 and the T1 threshold trigger is checked again for 3-fold coincidence above 3,2 $\mathrm{I}^{\text {est }} \mathrm{VEM}$ threshold and promote to T2 trigger. The rate of the T2 trigger is around $20 \mathrm{~Hz}$. All T2 triggers are sent to the CDAS in order to do the correlation check between tanks.

The T3 triggers are evaluated by the CDAS event builder. The T3 trigger checks the correlation in time and space for all stations sending T2 triggers. The coincidence of 3 adjacent tanks (minimum compactness requirement: one tank has one of its nearest neighbors and one of its second nearest neighbors triggered) that have passed the ToT condition is required for the $\mathrm{T} 3$ trigger.

The left side of the figure 14 shows a possible 3 -fold configuration. It selects principally physical events and the 90\% of the chosen events are showers. Another T3 trigger in this level requires a 4-fold coincidence (moderate compactness requirement) with the condition that one tank with $\mathrm{T} 2$ trigger can be up to $6 \mathrm{~km}$ far from others within a reasonable time window. This trigger is necessary in order to detect horizontal showers. The right side of the figure 14 shows an example of the 4-fold configuration.
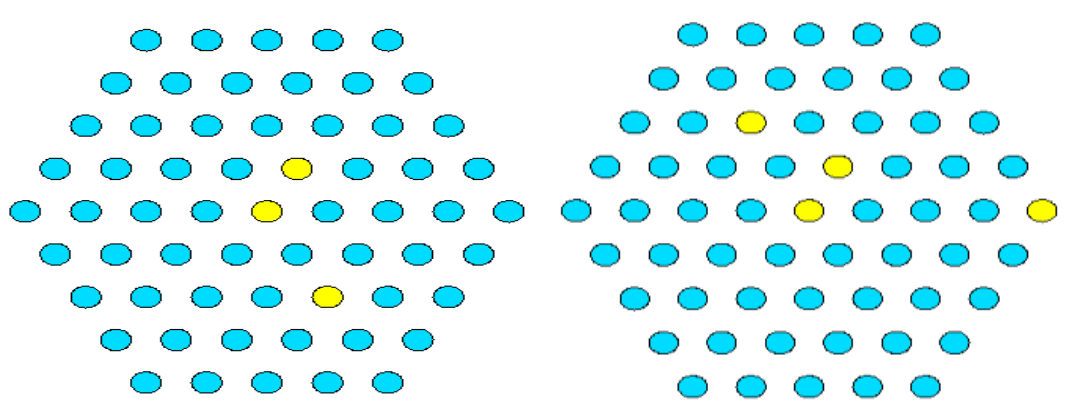

Fig.14. 3-fold and 4-fold coincidence for the T3 trigger level (triggered tank: yellow color)
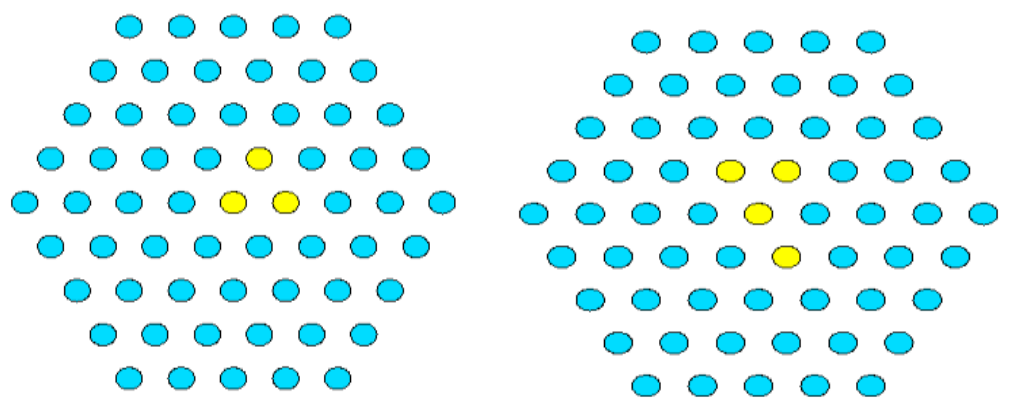

Fig.15 3 ToT and 4C1 configurations for the T4 trigger level (triggered tank: yellow color) 
In order to select only showers from the $\mathrm{T} 3$ data, a physical trigger T4 is necessary. This physical trigger is used offline in order to select events with zenith angles below 60 degrees. Two behaviors of the vertical showers are used in order to do the selection: the 3 ToT compactness configuration of the triggered tanks or $4 \mathrm{C} 1$ compacity configuration (at least one fired station has 3 triggered tanks out of its 6 nearest neighbours). Those configurations are shown in the figure 15.

Between the events that pass the T4 trigger, only those events that can be reconstructed with a well-known energy and an angular precision will be used for the T5 quality trigger. It requires that the tank with the highest signal must have at least five active tanks among its 6 nearby neighbors and the reconstructed shower core must be within an equilateral triangle of active stations [25].

On other hand, large fluctuations of ToT trigger rate, produce an increase of ToT trigger rate. It changes the threshold levels for the trigger system, affecting in this form the step 1 and 2 of the calibration procedure of Surface detector. Because the conversion of the signal of the PMTs into VEM units (step 3) depends of the step 2, so the fluctuations of the ToT trigger rate affects in this way to the calibration of the detector. In the figure 16, the left plot is the plot of the trigger efficiency versus the VEM signals and the right plot is the trigger efficiency versus the energy (for Iron, Proton, and Gamma rays).
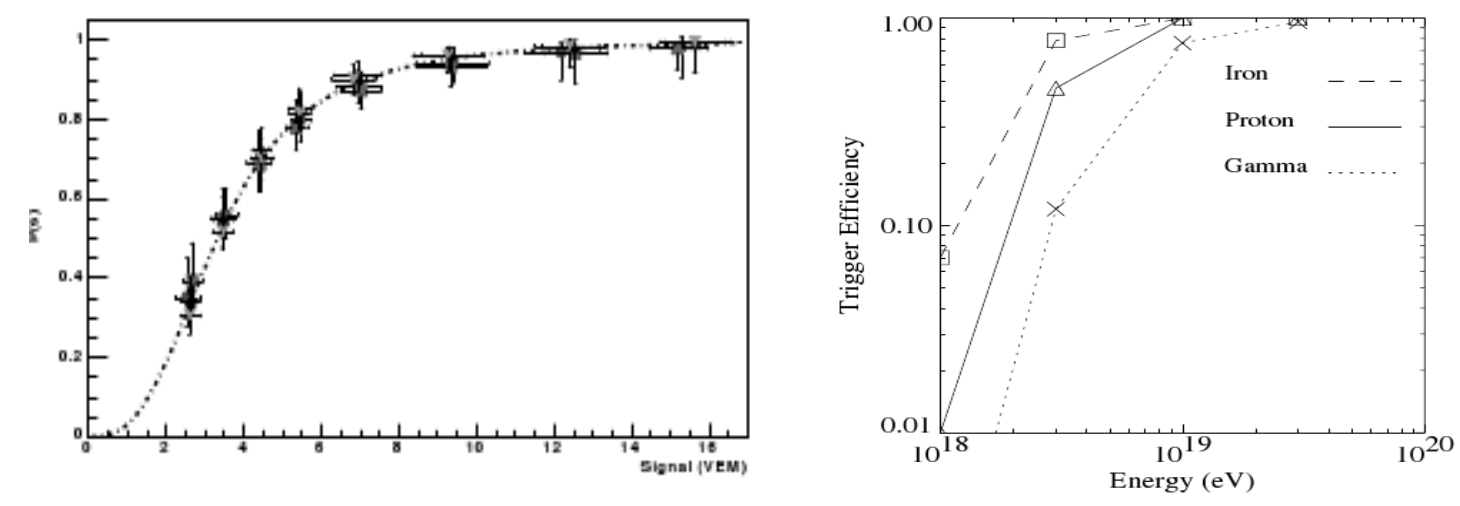

Fig.16. Probability for a local station to pass the trigger system in units of VEM and in units of eV (trigger efficiency). The right plot shows separately the trigger efficiency for Iron, Proton and Gamma rays [25], [27]

It is possible to observe in the figure 16 that for energies above $10^{19} \mathrm{eV}$, the trigger efficiency is $100 \%$ saturated (VEM signal $>>10, E>10^{19} \mathrm{eV}$ ). Because the energy reconstruction is done in the region of around $10^{19} \mathrm{eV}$, trigger rate fluctuations does not affect it because the trigger efficiency is saturated [25].

\subsection{The Central Data Acquisition System (CDAS)}

The Central Data Acquisition System (CDAS) was designed to assemble the triggers of the SD, to form the third level trigger of the detector, to allow the control of these detectors and to organize the storage of data. The data that are sent by the LS to CDAS correspond to some sections (streams):

- Trigger stream: mainly composed of the local trigger of the SD.

- Control and Service Stream: it concerns the control of the configuration of the LS from the CDAS and also to 
download and test the local station software.

- Data Stream: FADC traces and the information of the calibration are sent to CDAS when an event T3 has been identified.

- Calibration and monitoring: The LS is sending information of the monitoring and calibration. The calibration is performed every minute and to send to CDAS every 7 minutes for monitoring [26].

\subsection{Monitoring Data}

In order to check the long term performance of the system and the accuracy of the online calibration, every 400 seconds (approximately 7 minutes) monitoring data are recorded from each Surface detector (SD) to ensure that they are performing as intended and also to understand the detector behaviour. The monitoring data are averaged over 20 readings made every 20 seconds. They are used to study the evolution of some parameters of the PMTs such as VEM Peak, VEM Area, VEM Area/Peak, Dynode signal, Anode signal, Dynode/Anode Ratio, ToT trigger rate as function of both time and temperature. The Monitoring is structured as allows: data from short term contained in the table MonitCalib and long term contained in the table LongTermMonitCalib. The table of MonitCalib has data available every 7 minutes. The following variables containing relevant information for the performance of the PMTs are available: Voltage, Temperature, VEM Area, VEM Peak, VEM Area/Peak, Anode, Dynode, Dynode/Anode Ratio, ToT trigger rate. The table of LongTermMonitCalib has data available from many months. One set of data is recorded every hour. This table has the following variables for the performance of the PMTs: Voltage, Temperature, VEM Area, VEM Peak, Dynode/Anode ratio.

\subsection{Observable Parameters in Monitoring Data}

The observable parameters of the PMTs that are present in the monitoring data and were used in the analysis of this chapter are: VEM Peak, VEM Area, VEM Area/Peak, Pedestal of the Anode, Pedestal of the Dynode, Dynode/Anode Ratio, ToT trigger rate, Voltage, Temperature. The chosen station for the analysis is the LsId=164, PMT3. The data shown here is from the short term Monitoring Calibration database.

\section{PMT Temperature}

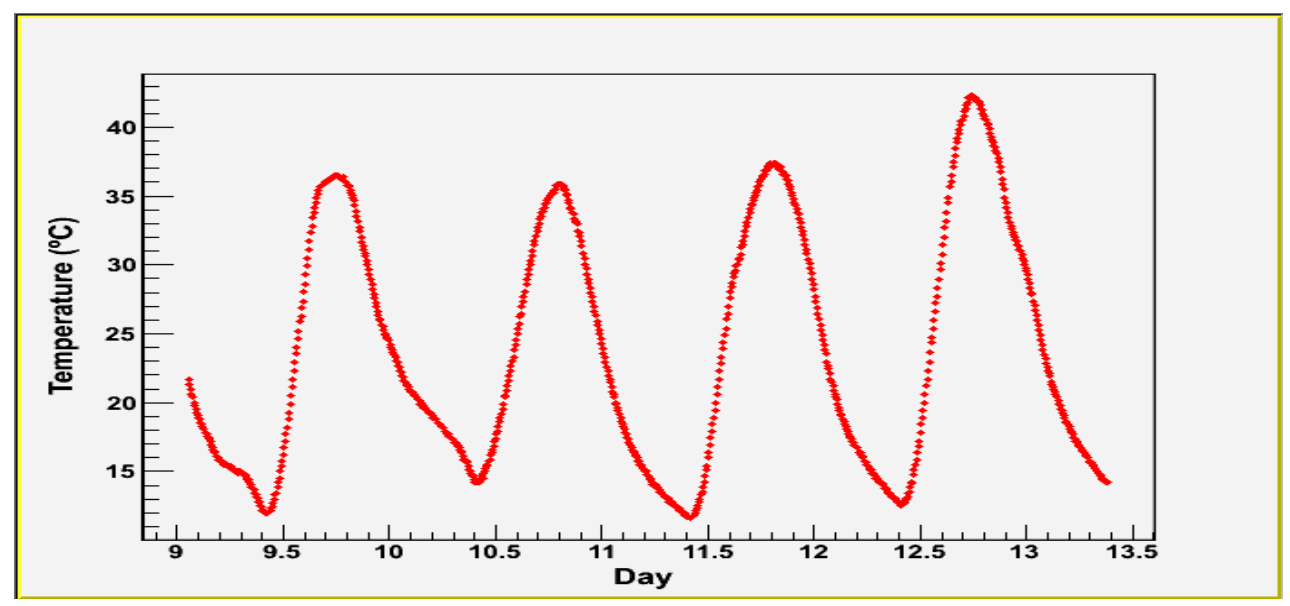

Fig.17. Plot of the Temperature of one PMT versus time 
At the base of each PMT the temperature is measured. This value is used to analyse the behavior of the parameters of the PMTs as a function of the temperature. The temperatures in Malague are in the range from $-20{ }^{\circ} \mathrm{C}$ to $60^{\circ} \mathrm{C}$ approximately with a large sinusoidal variation between temperatures at day and night. In the next figure, the pattern of the temperature signal for one PMT is shown. The variation of the temperature between day and night is clearly visible.

\section{VEM Peak}

The VEM Peak is defined as the height of the signal of a vertical muon crossing the centre of the tank. A plot of a typical behavior for one PMT is given in the next figure.

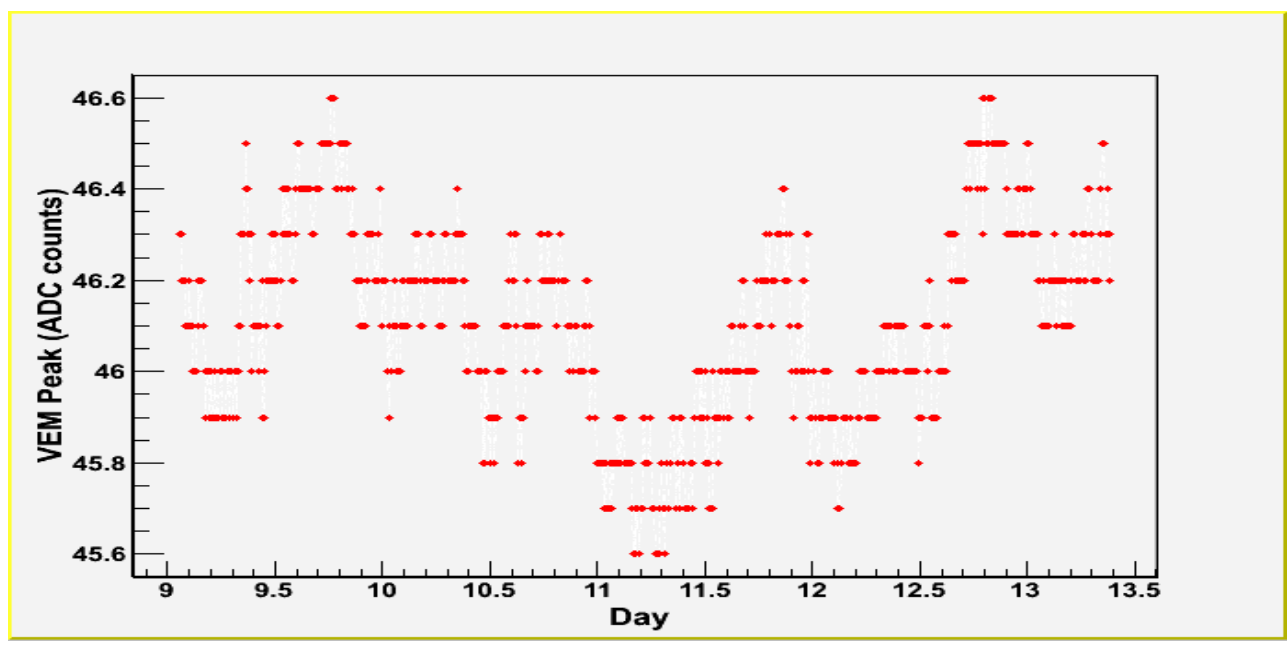

Fig.18. Plot of the VEM Peak versus time

The average value is around $46 \mathrm{ADC}$ counts with fluctuations in the order of $2 \%$. A small variation of the signal (around $1 \mathrm{ADC}$ count) due to the temperature is observable in the figure.

\section{VEM Area}

The VEM Area is defined as the average area of a VEM signal. Therefore, the total charge deposited by a VEM signal is the VEM Area. In the figure 19, the general behaviour for one PMT is given.

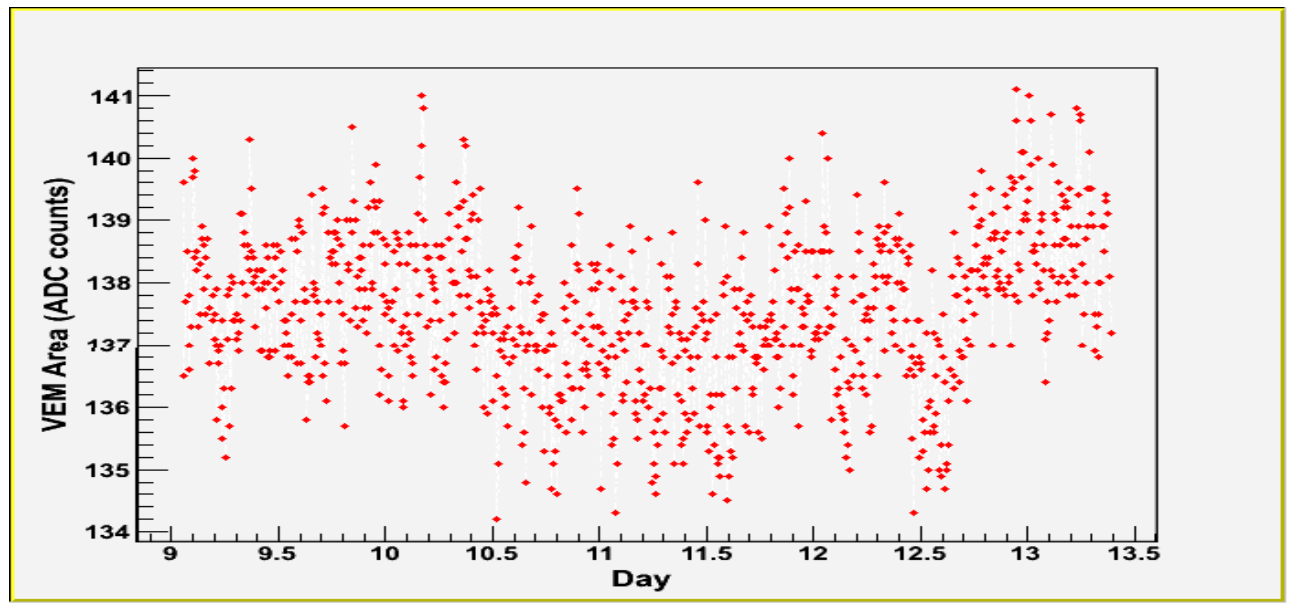

Fig.19. Plot of the VEM Area versus time 
The average value is around $138 \mathrm{ADC}$ counts with fluctuations of around $2 \%$. In the figure 19 is possible to observe small variations in the order of 3 ADC counts, which is mainly due to temperature variations. The VEM Peak appears to be more sensitive to changes of the temperature than the VEM Area.

\section{Ratio of VEM Area over VEM Peak}

The VEM Area/Peak is a measure of the signal width. This ratio depends on the properties of the water and the Tyvek reflectivity of the tank. The behaviour of this variable is given in Figure 20.

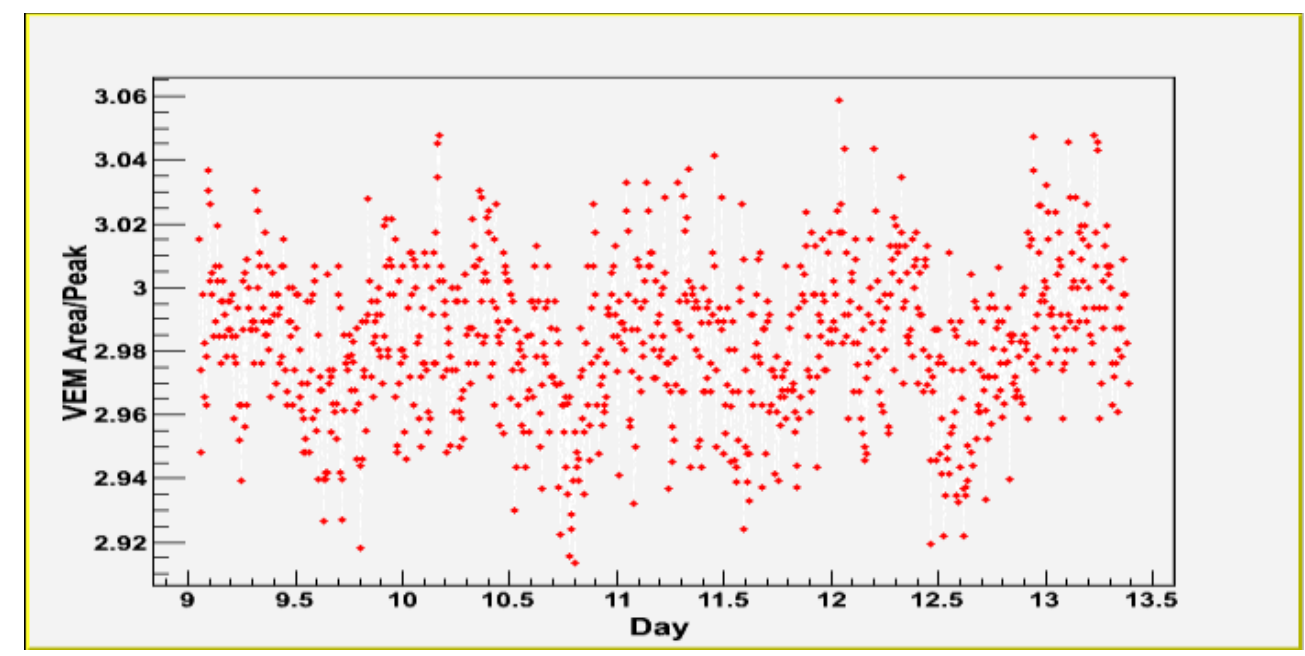

Fig.20. Plot of the VEM Area/Peak versus time

The average of VEM Area/Peak is around 3 with fluctuations also of about $2 \%$.

Anode pedestal

The anode pedestal is the signal of the anode at the output of the amplifier when the PMT does not have an input signal. A typical plot of this variable for one PMT is given in the figure 21.

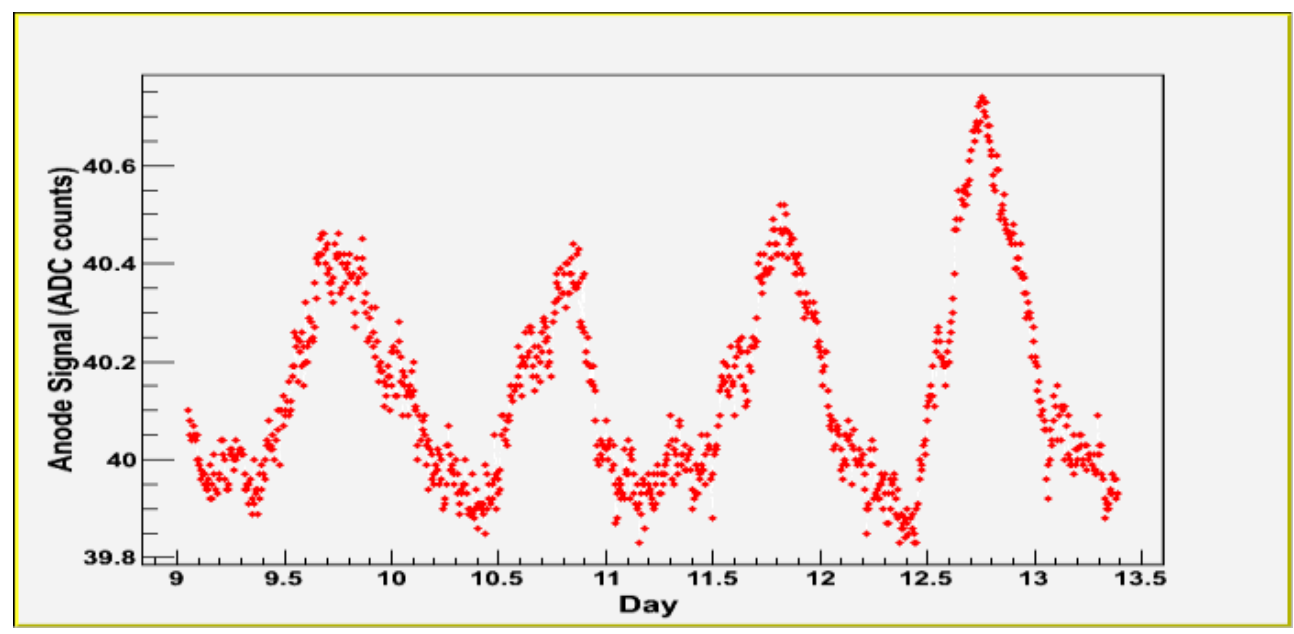

Fig.21. Plot of the Anode Pedestal versus time

The average value for the anode pedestal is around 40 ADC counts and the fluctuations are around $1 \%$. It is possible to observe in the figure small variations of sinoidal form of around 1 ADC counts. If we compare this plot with the figure 17 , we observe that there is a strong correlation with the temperature. 


\section{Dynode pedestal}

The dynode pedestal is the signal of the last electrode before the anode at the output of the amplifier when the PMT does not have an input signal. The plot of this signal for one PMT is given in the figure 22. It shows the same behavior with the temperature as the anode pedestal, but the sinoidal variations are larger than the variations of the anode pedestal.

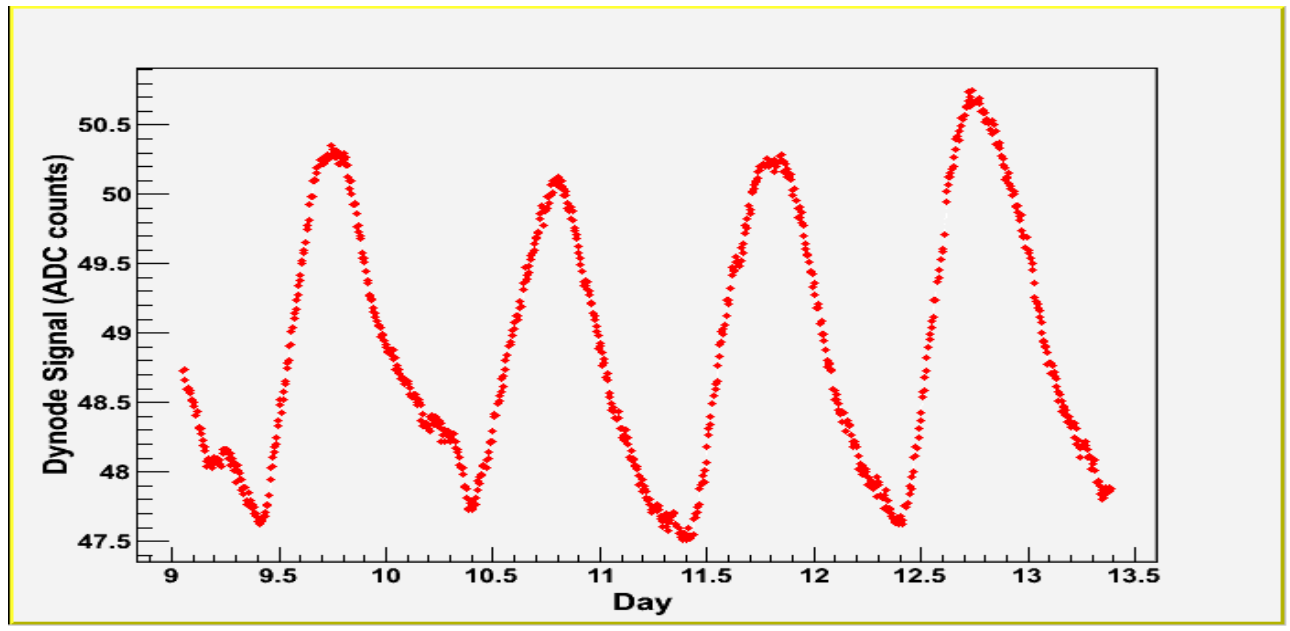

Fig.22. Plot of the Dynode Signal versus time

The value for dynode pedestal is around 49 ADC counts with fluctuations of around 3\%. Also for this signal the strong linear correlation with the temperature is observed.

\section{Dynode/Anode Ratio}

The Dynode/Anode Ratio is defined as the ratio between the amplified signal (32 times) from the last Dynode stage to the signal at the Anode. This parameter is used in order to obtain the corresponding calibration of one VEM in the anode channel. For tanks close to the shower core, the dynode readout is saturated.

For these events the signal measured on the anode should be used. Therefore, the accuracy of the signal size and the energy of the event depend of this parameter. The typical pattern for one PMT is given in the figure 23.

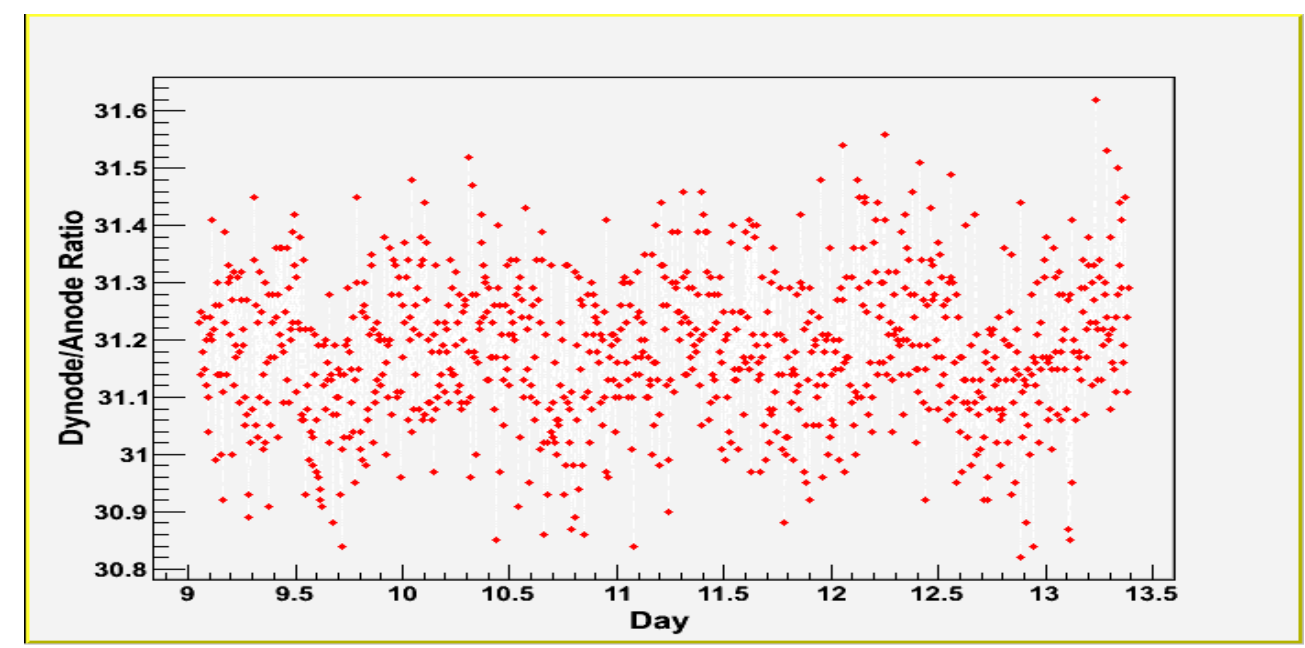

Fig.23. Plot of the Dynode/Anode Ratio versus time 
The average value for the signal of the Dynode/Anode is around 31 with fluctuations in the order of $2 \%$.

\section{ToT trigger rate $(\mathrm{ToT})$}

The ToT trigger rate importance comes from its capability to reduce the single muonic background significantly. So, to fulfill the ToT trigger the signal is required to be above a threshold, with a given coincidence between PMTs, for a minimum number of bins within a sliding window. The nominal ToT trigger rate condition needs that the signal has to be above 0.2 VEM in 2 PMTs for a minimum of 13 bins within a time window of 120 bins. The ToT trigger rate has a strong dependence on the water quality because as the quality of the water changes, the width of a muon signal and consequently the ToT trigger rate changes. In the figure 24 the current behaviour of this parameter is shown. The typical value of the ToT trigger rate is $0.2-0.3 \mathrm{~Hz}$.

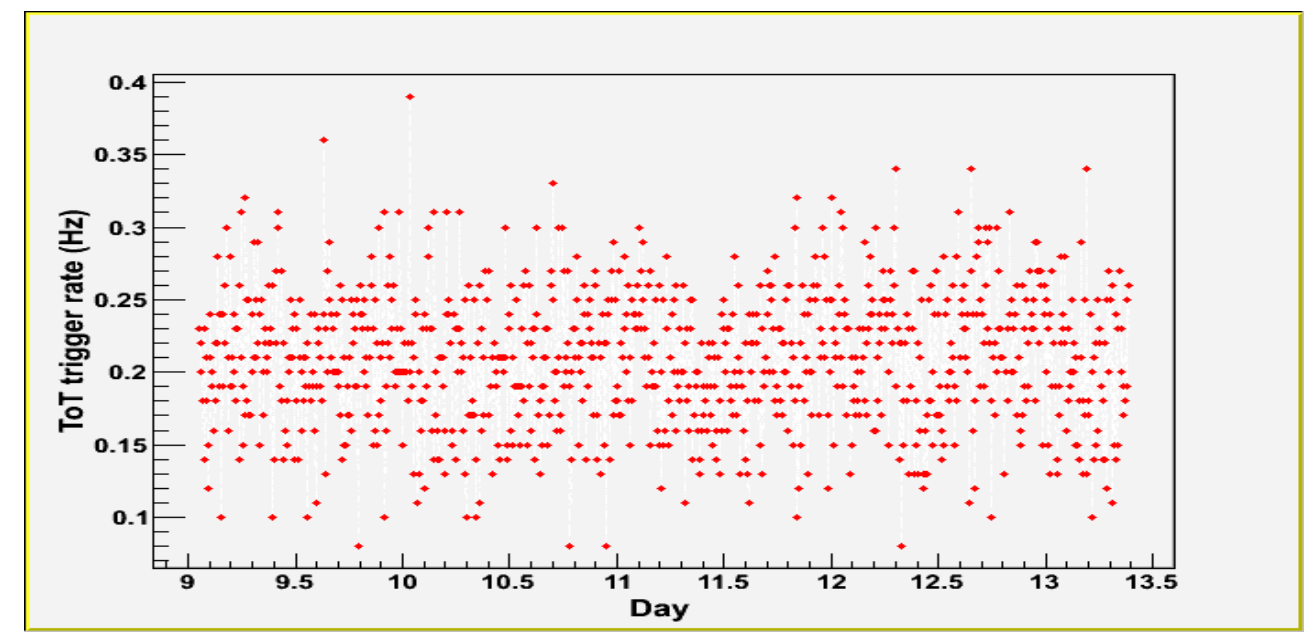

Fig.24. Plot of the ToT trigger rate versus time

\section{PMT High Voltage}

The high voltage of the PMT is the readout of the positive voltage applied to the PMT anode. This value should be stable with slight correlation with the temperature. The figure 25 shows a typical behaviour of this signal for one PMT.

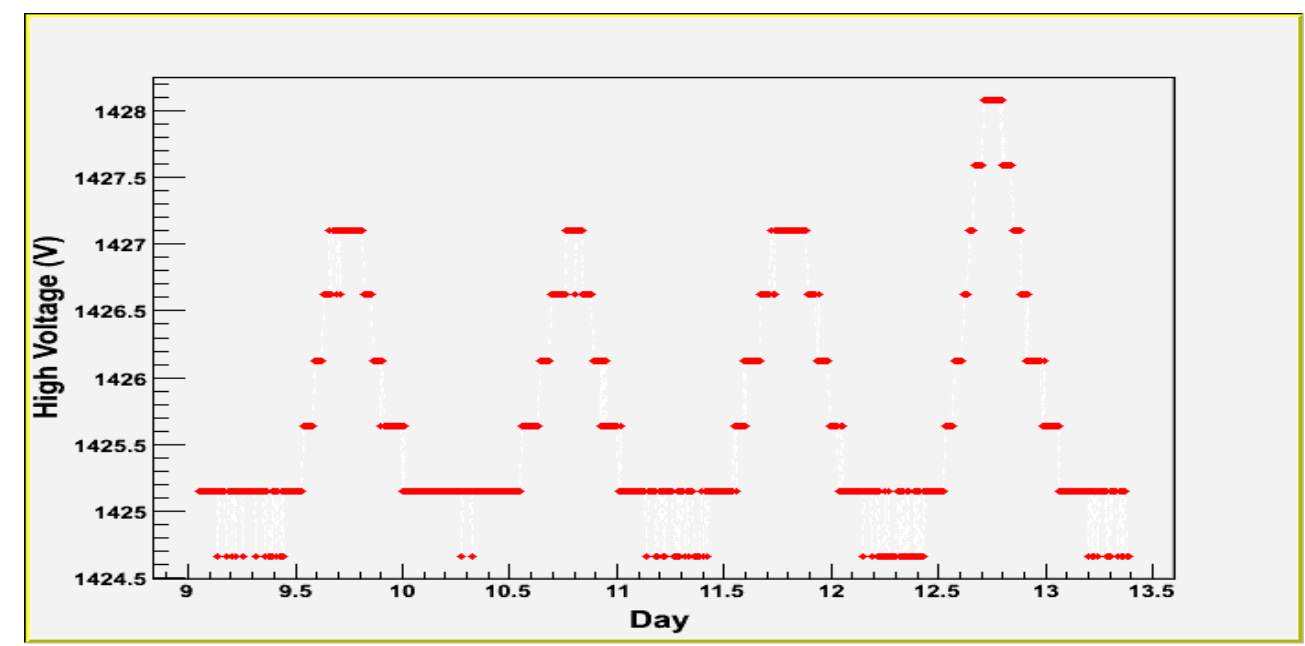

Fig.25. Plot of the High Voltage of the PMT versus time 
The High Voltage applied to the PMTs is in the range between 1000 and 1600 V. For some PMTs the High Voltage is changed in order to adjust (recalibration) the VEM value of the PMT. The High Voltage of the PMT is supplied by a battery of $+12 \mathrm{~V}$. Therefore, changes in the voltage of the battery also can affect the voltage output.

\subsection{Alarms Creation}

The aim of the monitoring data is to find problems with the data taking as soon as possible. The optimal way is to identify problems before they diminish the quality of the data. In the near future several classes of alarms have to be created, from those which might cause problems to those which need immediate action by one of the shifter. Therefore, after that the analysis of the respective parameters of the problem is done, it is necessary to define the respective alarm for them.

\section{Language Programming}

For the creation of the Monitoring Alarm, the following language programming are used:

* It is used C++ compiled language. It has the advantages: availability, portability, speed, generality, reusability and object orientation. Object oriented programming is a strategy for simplifying the programming task.

* MySQL database management system. It is needed in order to add, access, and process data stored in a computer database.

* ROOT: an object-oriented framework. It is aimed to solve the data analysis challenges of high- energy physics. In this framework the basics services like graphics and histograms are provided.

The alarm code should be written in efficient way. So, it is recommended to have an elaborate and efficient MySQL request in order to have a fast MySQL request and to reduce the $\mathrm{C}++$ code. Thus, it is necessary to use the large panel of options offered by MySQL. In general, the condition of the alarm should be in the request itself.

\section{Free Disk Alarm}

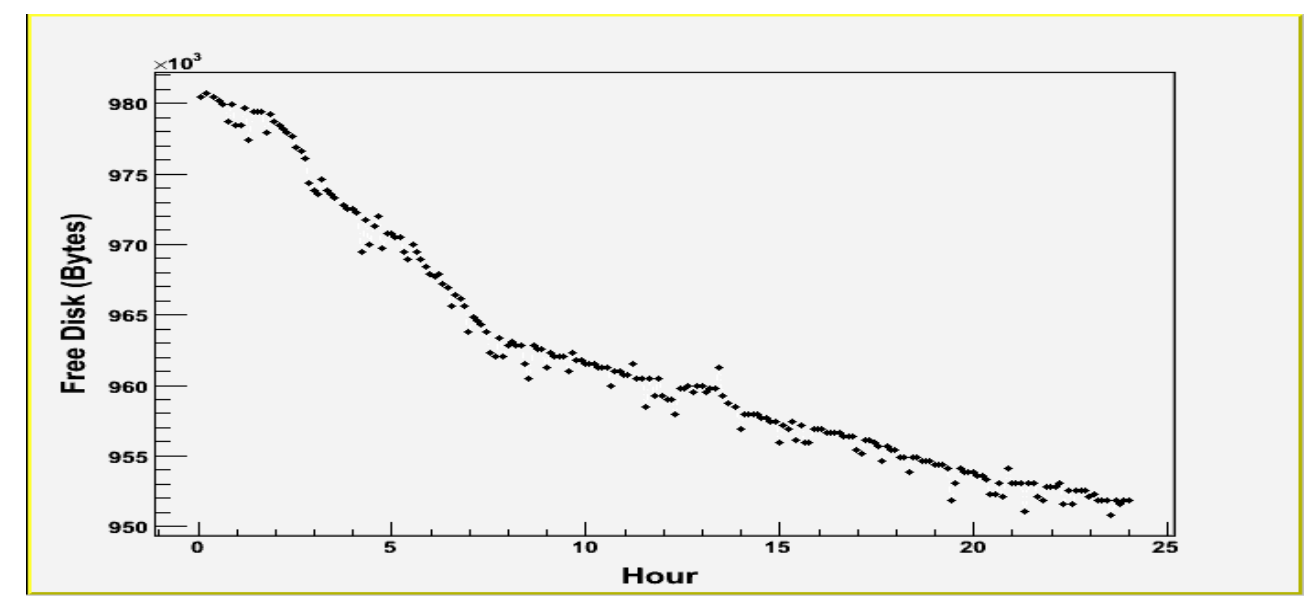

Fig.26. Plot of the Free Disk versus time (LsId: 1200, date: 10.12.2007)

In this section, the procedure of the alarm creation is described with the example of the Free Disk alarm. This 
alarm was created and installed in Auger Online Monitoring. The disk of each local station is a RAM disk with a capacity of 2 Mega Bytes [30]. The next figure shows one example of the behaviour of the Free Disk space for one local station as a function of time.

As too little space can create problems in data taking, stations with values of the Free Disk below a given threshold should trigger one alarm. The figure 27 shows distribution of the Free Disk variable for 8 days (6-13) in December 2007 for all the local stations.

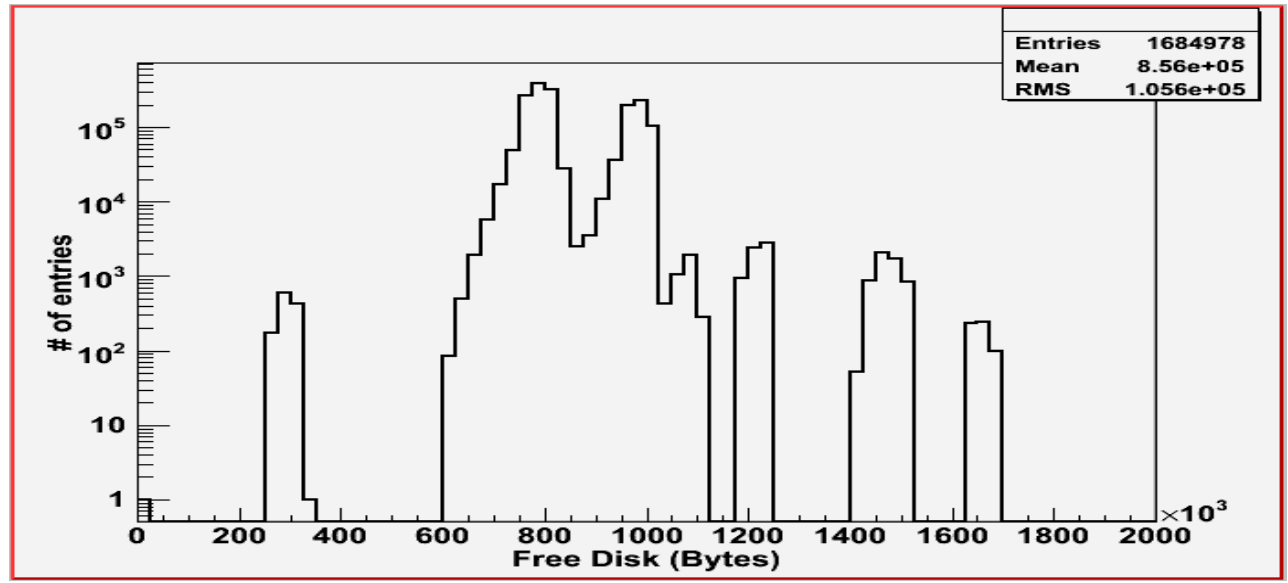

Fig.27. Distribution of the Free Disk for all local stations

From the distribution, it is possible to observe that nearly all stations have values of the Free Disk of more than 600000 Bytes. This value corresponds to the chosen threshold. The algorithm of the alarm is:

If for one local station the Free Disk< 600000 Bytes $(30 \%)$ the alarm is triggered. If the alarm is triggered, shifter action should be to report to the Surface Detector Team that there is a local station with too low free disk space.

\section{High PMT gain change alarms}

The procedure of the alarms for high PMT gain change can be realized in the same way that the Free Disk Alarm was done. The only differences are the conditions for the alarm, the selected variables and the threshold values. The RMS of the VEM Peak and Dynode/Anode Ratio are the variables chosen for the alarms. The conditions and the thresholds values are:

A condition for the bad behaviour (High PMT gain change) of the PMTs was researched and so, a threshold of:

RMS (VEM Peak) $>1$ ADC count and RMS (Dynode/Anode Ratio) $<0.5$ should be used in order to select those PMTs.

\section{Wiki information}

For each alarm a wiki entry is created. The wiki information should contain all the information about the alarm, the threshold values, the algorithm of the alarm, plots, histograms, physical information, etc. Also it is necessary to specify which shifter action has to be performed if the alarm is triggered. Further information as the bibliography and contribute notes where it is possible to find more information concerns to the alarm is necessary to document. 


\section{Muon production and decay}

The muons form the muonic component and they decay with a lifetime of $2,2 * 10^{-6} \mathrm{~s}$ into neutrinos and electrons. Muons are charged particles that are created at high attitudes at the earth atmosphere. Due to their high relativistic velocities close to the speed of the light, many of them reach the earth surface. The number of muons in the evolution of the shower is around 1\%. Muons are enough energetics (hard component), rarely interact and only decay under weak interactions [35]. It causes that they can reach the sea level and penetrate deep underground, but it is necessary to consider the relativistic effects. Therefore, about $80 \%$ of the charged particles at the sea level are muons. Most of them get trapped in the scintillator detector or water Cherenkov detector at the earth surface, and some even stop and decay there. Others continue through the detector and decay sometime later [35]. The muons were first discovered at cosmic rays about 1936 by Anderson, Nedermeyer and others. Before, Yukawa postulated a particle approximately the muon mass as quanta of the field responsible for the nuclear forces. However, it turned out that the particles of intermediate mass (mesons, pions) first seen in cosmic rays were not the particles postulated by Yukawa, because they do not interact appreciably with the nucleons. For historical reasons, the muon is sometimes called improperly the mu meson but the word meson should be reserved only for bosons. The charge of the muons (positive and negative) is equal in magnitude to the charge of the electron [35]. There are no neutral muons and the muons are usually obtained as a decay product of the pion according to the reactions:

$\pi^{+} \rightarrow \mu^{+}+v_{\mu}$

$\pi^{-} \rightarrow \mu^{-}+\overline{v_{\mu}}$

For other hand, free muons decay into electrons and neutral particles. The electron spectrum is a continuum and thus more than one invisible neutral partner is required to conserve energy and momentum [35]. The form of the spectrum agrees with the prediction of the reaction:

$\mu^{+} \rightarrow e^{+}+v_{e}+\overline{v_{\mu}} \quad \mathrm{m}_{\mu}=207 \mathrm{~m}_{\mathrm{e}}=105.7 \mathrm{MeV} / \mathrm{c}^{2}$

$\mu^{-} \rightarrow e^{-}+\overline{v_{e}}+v_{\mu}$

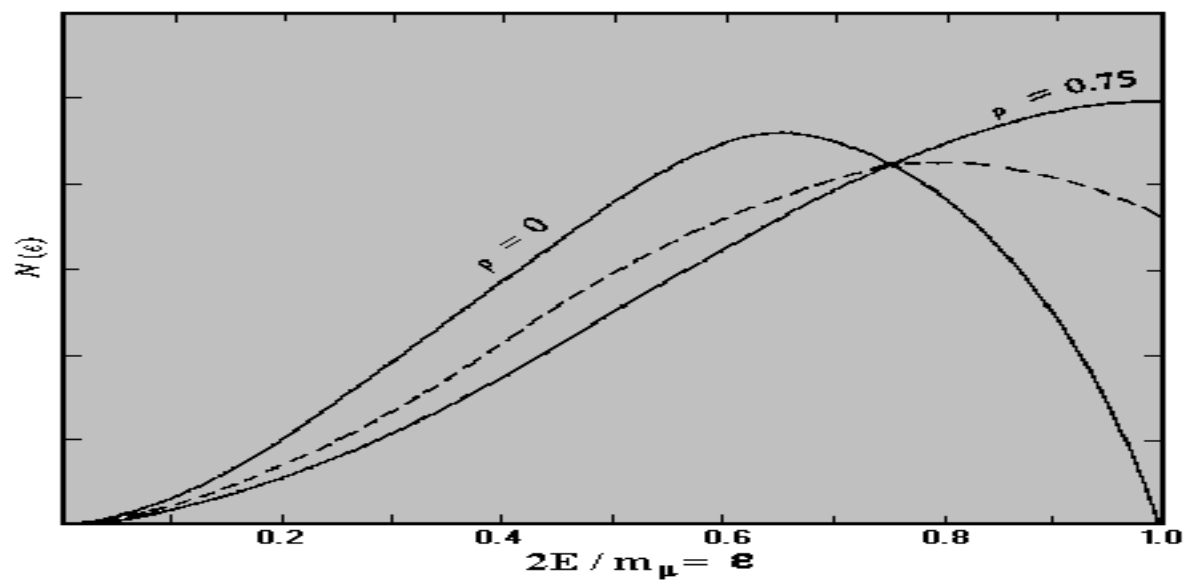

Fig.28. Energy spectrum of the electrons from muon decay 
The interaction is classified as weak interaction and it is completely similar to beta decay. About the two neutrinos, one is believed to be of the kind found in beta decay, and the other of the kind found in pion decay [35]. The continuous energy spectrum of the electron decay has the form indicated in the above figure.

The energy of the electrons is greatest when the two neutrinos (electron neutrino and muon neutrino) escape in the same direction, opposite to the direction of the electron. The probability of this occurrence at this case is 0 . Pauli Principle prevents that two identical neutrinos have the same quantum state and move at the same direction [35].

Therefore, we conclude that in muon decay two different neutrinos are emitted. The decay of the muon by weak interaction is particularly important because none of the involved particles is subject to strong interactions. Thus, we have a clean case to test the weak-interaction theory [35].

For other hand, negative muons stopped in matter may decay freely, but they can also interact with the nuclei of the stopping substance and disappear by capture process. Furthermore, Pancini and Piccioni at 1947, demonstrated that the muons interact only weakly with the nuclei and established the distinction between pions and muons. The probability for a muon capture increases with the fourth power of the nuclear charge number [35]. Besides, when the negative muons are stopped in a substance of a given $\mathrm{Z}$, their mean life is:

$\frac{1}{\tau}=\frac{1}{\tau_{\mu}}+\frac{1}{\tau_{c}}$

The first term on the right corresponds to the free decay of the muon and the second term is due to the disappearance caused by the interaction with the nucleus of the stopping medium (electronic capture). For $\mathrm{Z}=11$, the two terms are almost equal but for $\mathrm{Z}$ greater or equal to 40 , the Bohr orbit is already inside the nucleus. Thus, the capture probability for this condition is very close to 1 . For light nucleus with charge number of 10 or less, negative muons will decay preferentially [35].

The decay probability for free muons at rest is the same for positive and negative muons. If negative muons are stopped in matter, the measured decay probability of this case is always larger than the decay probability for free positive or negative muons. At this research, muons are stopped in a liquid scintillator which consists essentially of carbon. The mean life for muons in carbon is equal to $2.03 \mu$ s. For light elements, it is equal to $2.20 \mu$ s [35].

\subsection{Scintillators and Photomultipliers}

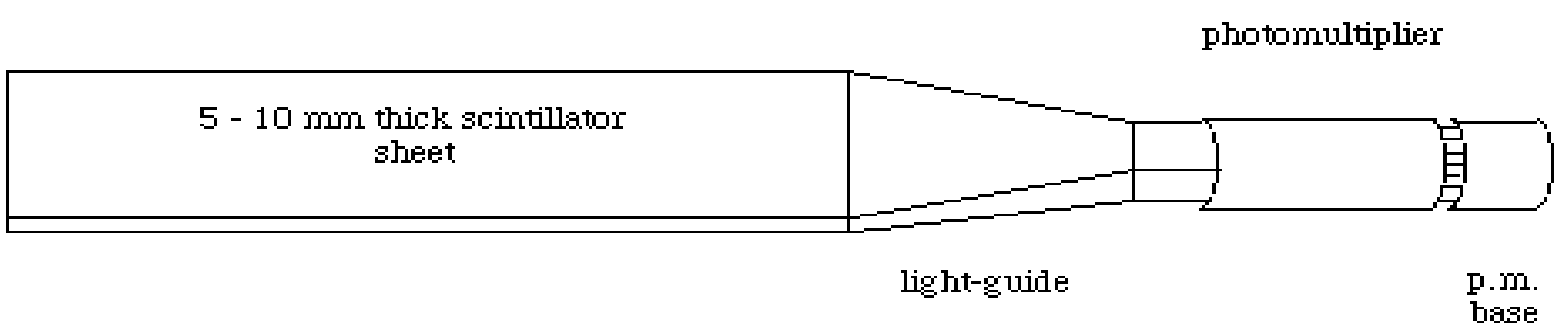

Fig.29. Schematic of a scintillator counter with the photomultiplier and light guide

The scintillator counter consists of a scintillator sheet, light guide, photomultiplier and the electronics at the photomultiplier base to read out the signal. The scintillator is composed of three components: primary scintillator, 
the wavelength shifter and the base material where muons and pions are stopped. There are inorganic scintillator (such as sodium iodide) and organic (such as a plastic like polystyrene). The charge particles pass through the material and excite electrons. They can de-excite emitting a photon. The primary scintillation light is emitted in the ultraviolet (UV) range preferentially. This photon would be exactly the correct energy to be reabsorbed by the material and the scintillator is not transparent to its own scintillation light. For this reason, the light is transferred to a wavelength shifter which absorbs the UV light and reemits it at a longer wavelength (green). It reduces the energy of the photon and so, the photon emitted is of longer wavelength with no enough energy to be reabsorbed. The scintillator sheet is highly polished and light is conducted by total internal reflection [35].

In this way, the emitted light can get out of the scintillator. The light must be transmitted from the scintillator to a sensor of the photomultiplier. It is done by a light guide and then it can be detected by the photomultiplier. The emitted light from the wavelength shifter is matched with the spectral sensitivity of the photomultiplier by this light guide [35].

Besides, the primary scintillator and the wavelength shifter are mixed with organic material in a plastic scintillator to form a polymerizing structure. At the liquid scintillators, the 2 components are mixed with an organic base. We need about $100 \mathrm{eV}$ to produce one photon in an organic scintillator. In our experiment, the liquid scintillator consists of p-therphenyle as primary scintillator, POPOP as wavelength shifter and Uvasol as base material plus the four photomultipliers coupled to the liquid scintillator via an air light guide. The photomultiplier converts the optical signal to an electrical signal and provides a high amplification [35].

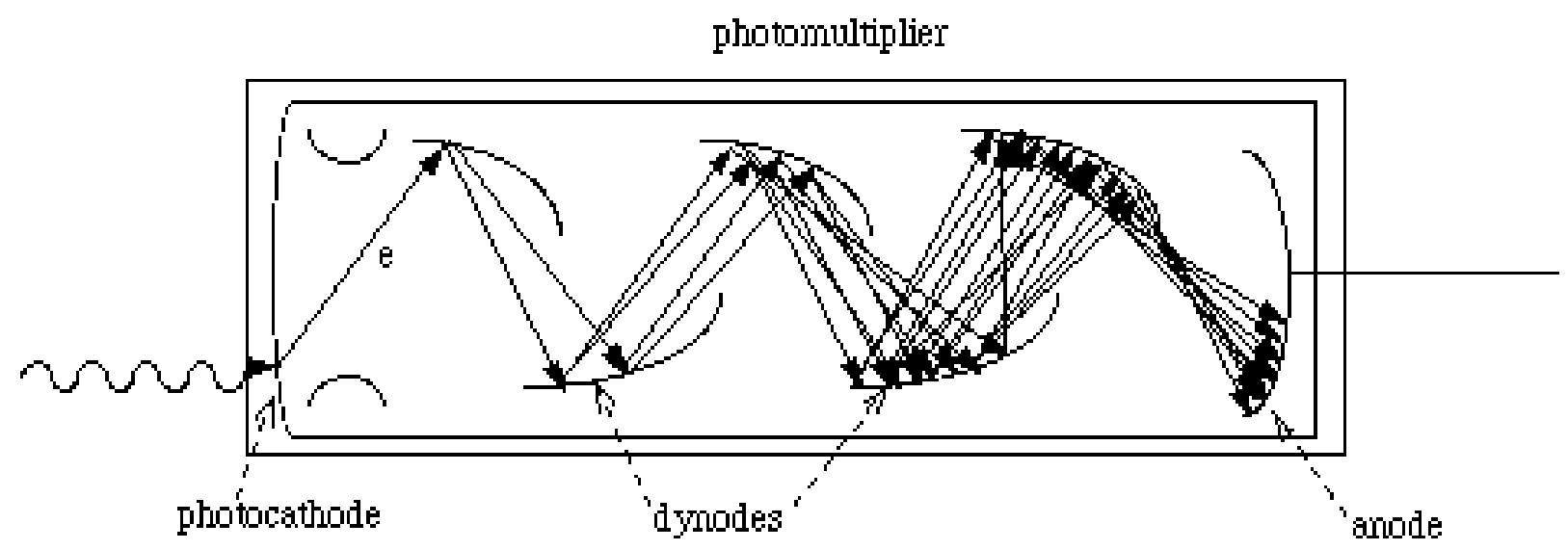

Fig.30. The photomultiplier

The photomultiplier consists of an evacuated glass envelope coated at one end with a photocathode made of alkali metals, which is maintained at a large negative potential. The photons liberate electrons (by photoelectric effect) from the cathode, which are accelerated towards a (less negative) dynode, and knock out a number of secondary electrons. This process continues until a large signal is collected at the anode.

The typical gain of the photomultiplier is of the order of $10^{7}$ and normally a pulse of a few $\mathrm{mV}$ per detected photon is produced. Finally, the connections to the photomultiplier are made through the photomultiplier base, which contains a chain of resistors to provide the correct voltages for the dynodes and cathode [35]. 


\subsection{Determination of the Mean Lifetime}

The deceleration of a muon or pion at the liquid scintillator gives a light signal which is recorded by the photomultiplier. We use a trigger for the time to amplitude converter and when the muon has come to rest no further light is emitted. A second signal is provided for electrons or decay products of pions which appears after a muon decay. This signal stops the time to amplitude converter. Thus, the time difference between the incoming stopping muon and the appearance of the electron is a measure of the average lifetime or the decay time of the individual muons [35]. By counting many events of the same kind, we obtain a time spectrum of the decay time given by the law of the radioactive decay:

$N=N_{o} e^{-\lambda t}$

Muons with decay at flight in the atmosphere are ignored by the circuitry. Besides, signals caused by radioactive background can be suppressed by adjusting the discriminator threshold. We can observe three components at the time spectrum: the decay of free positive muons, negative muons and chance coincidences [35].

Chance coincidences can be measured at very large decay. It is very small but they have to be subtracted. Also, It is obtained contributions from decaying positive muons and decaying negative muons which have a different lifetime. For other hand, negative muons can also be captured. Because the sum of two exponentials with different slopes is not an exponential again, it makes necessary a correction due to muon capture using the value for the stopping negative muons in carbon of 2.06 us [35].

In similar way, the spectrum for pion decay contains three contributions: chance coincidences (uniform distribution), muon decay (which is uniform over a time range of $200 \mathrm{~ns}$, and we can use here an average effective common lifetime for $\mu^{+}$and $\mu^{-}$), pion $\pi^{+}$decay (because $\pi^{-}$always is captured by the nuclei). The chance coincidence, and muon decay are subtracted and it is analysed only the pion decay [35].

\subsection{Electronic logic for the measurement of the decay time of muons and pions}

High energetic particle passes through the liquid scintillator generates photons in the ultra-violet range of the light spectrum. The scintillator emits light in the visible range and then, some of these photons go to the photomultiplier tubes (PMTs).

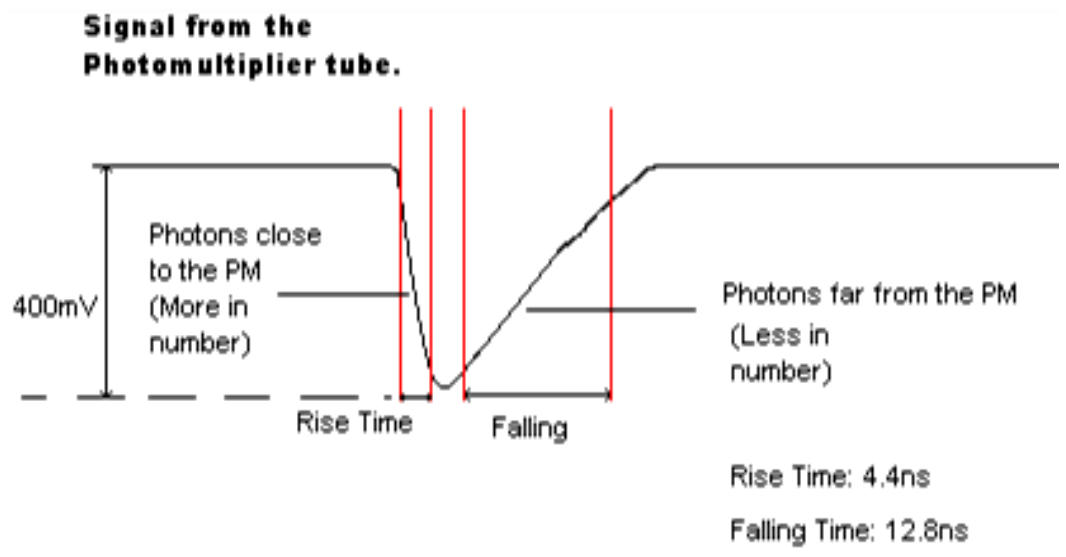

Fig.31. Signal coming from the photomultiplier 
The PMTs operate according to the photoelectric effect, where energy from photons hit a metal surface. Then, the photons are absorbed by the electrons at the metal. These electrons gain enough energy to be "knocked out" of their normal location at the atoms of the metal. Thus, these traveling electrons can be detected as an electric signal. The photomultiplier tubes are powered by a high voltage power supply. The above figure shows a signal coming from a photomultiplier tube as it is seen at the oscilloscope [35].

It is possible to observe that the signal consists of two parts: one coming from the photons which are generated at the scintillator close to the photomultiplier tube (called rise time) and a longer part which is due to the photons generated far from the photomultiplier tube (called falling time). The signals from each of the 4 photomultipliers go to the discriminators (which have a threshold of $30 \mathrm{mV}$ or rather $-30 \mathrm{mV}$ since the signal is negative) and give the signals a width of 10-12 ns. Then, the pulse generator transforms the signal from the photomultiplier tubes and so, all the signals have a constant size and shape [35].

Then, the standard signals are sent to the Logic Unit which is set up to look for two signals coming at the same time (coincidence). When two coincident signals are received by the Logic Unit, one signal is sent out to the Scaler. Because there is only one pulse generator at this research, the coincidence level (majority condition) is set to one. The gate generator selects the time window where the events are to be counted. So, it is $200 \mathrm{~ns}$ for pions and $10 \mu \mathrm{s}$ for muons. The start and stop signals will have to happen within this time window in order to be counted. Furthermore, there is a time delay of $32 \mathrm{~ns}$ which means that the stop and the start signals are only allowed after such time intervals. The operation of the detector is improved with NIM electronics for counting instead of manually counting pulses at the screen. After the NIM electronics, the signal goes to the time to amplitude converter (TAC) and then, it finally goes to the multichannel analyzer (MCA) [35].

\subsection{Experiment results}

Since the data read from the computer is of the form counts versus channel, it is required to find out the time-channel number calibration.

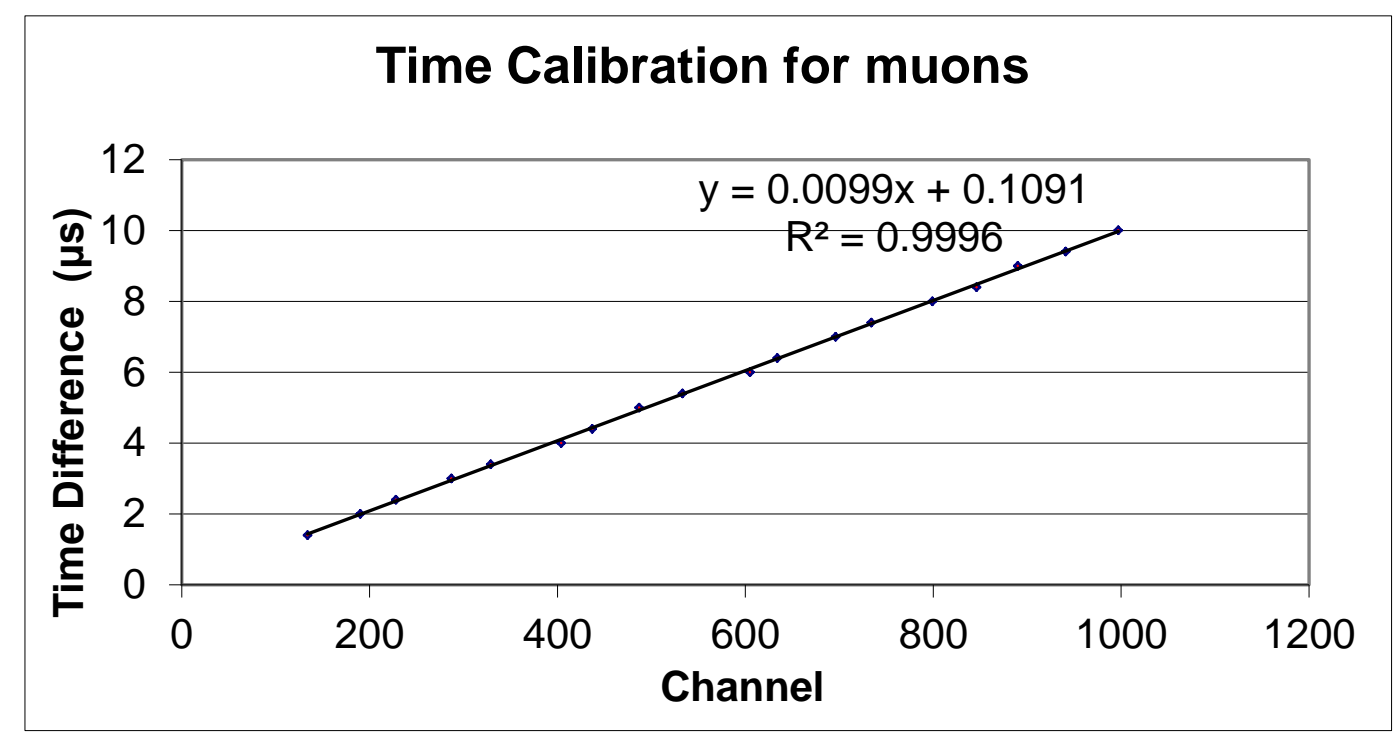

Fig.32. Time calibration for muon counting 
From the electronic circuit diagram, the gate generator defines the time window at which the stop signals are accepted. This time window is different for the measurement of muons and pions. For muons, it was set to $10 \mu \mathrm{s}$. Then a time calibration was performed using the time analyzer, a pulse generator and an oscilloscope. By setting different time delays from approximately $1 \mu$ s up to almost $10 \mu \mathrm{s}$ (the time window for muons), the corresponding channel numbers for each time difference were obtained [35]. The next figure shows the time calibration for muons. As it can be seen at the figure 32, the relation between the channel number and the time is linear. Then, this relation was used to convert the counts vs channel number spectrum into a counts vs time difference spectrum [35]. The figure 33 shows the counts vs the time difference spectrum.

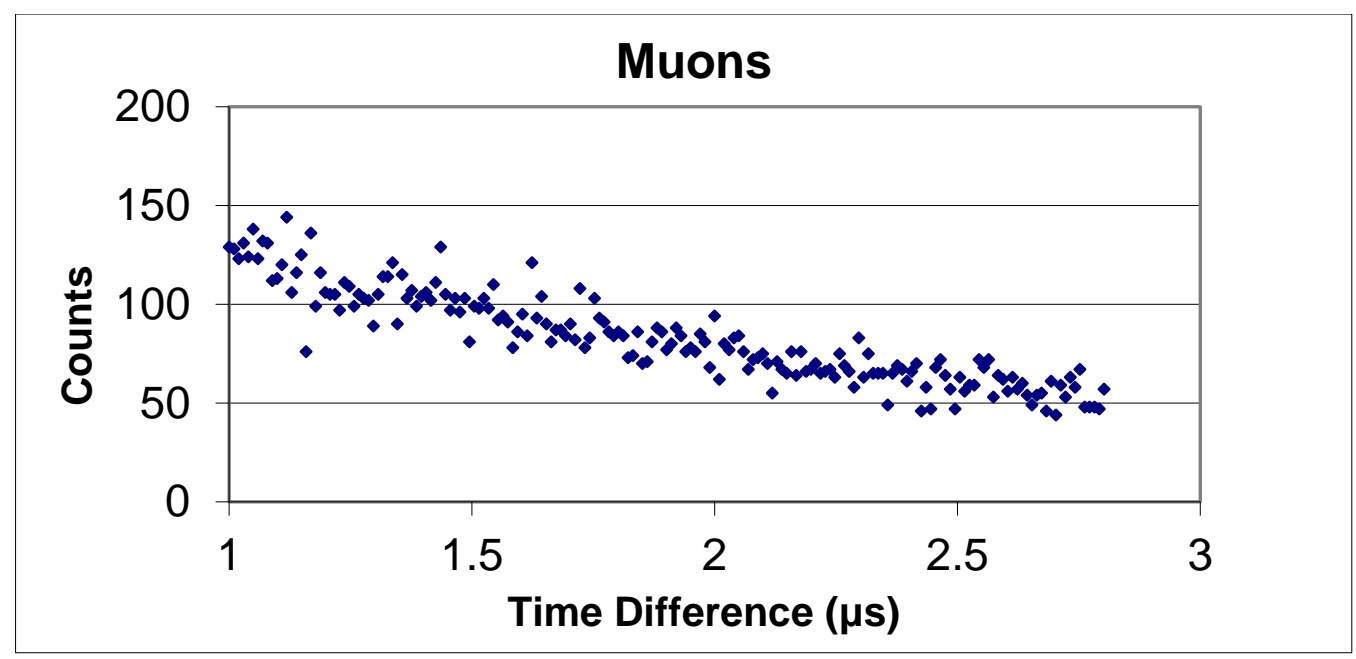

Fig.33. The spectrum obtained for the muon decay events

The muon decay should follow an exponential decay $\left(N=N_{o} e^{-\lambda t}\right)$ with the time in a similar way as a radioactive source. However, here we don't have the same source to measure photon fluxes as time goes. It is because either the muon decays or doesn't. The following figure 34 shows the natural logarithm of the counts (which represent events of muon decay) versus the time difference.

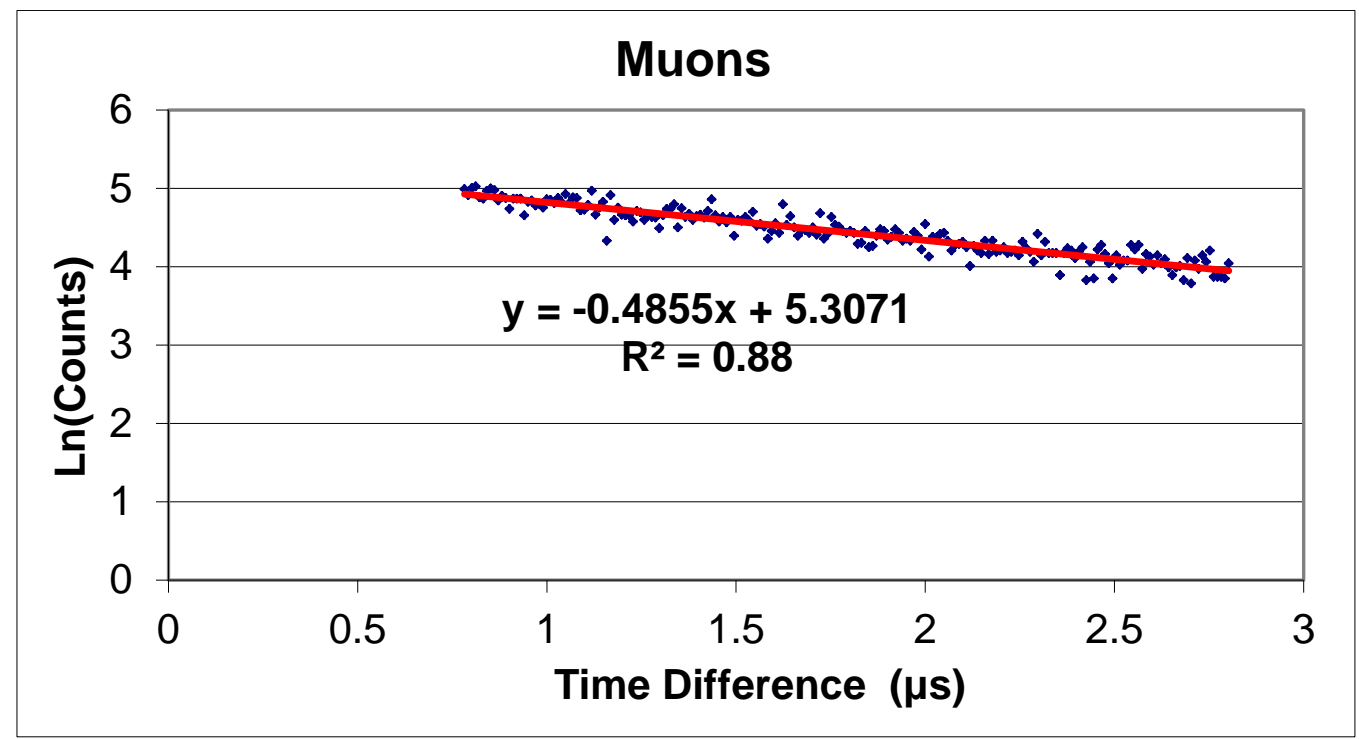

Fig.34. The natural logarithm of the counts versus the time difference for muons 
The first thing to notice is that the data points follow a straight line. Therefore, it is concluded that the counting of the muon decay events is indeed exponential in shape. In this way, the expected exponential distribution can be best seen and analysed [35].

$N=N_{o} e^{-\lambda t} \quad \ln (\mathrm{N})=\ln \left(\mathrm{N}_{\mathrm{o}}\right)-\lambda \mathrm{t} \quad \mathrm{y}=\mathrm{A}+\mathrm{Bt} \quad \mathrm{B}=-\lambda \quad \mathrm{y}=\ln (\mathrm{N}) \quad \mathrm{A}=\operatorname{Ln}\left(\mathrm{N}_{\mathrm{o}}\right)$

The best line $\mathrm{y}=\mathrm{A}+\mathrm{Bt}$ fitted to the data points is:

$\mathrm{y}=5,3071-0,4855 \mathrm{t} \quad \ln \left(\mathrm{N}_{\mathrm{o}}\right)=5,3071 \quad \mathrm{~N}_{\mathrm{o}}=201,76 \quad \lambda=0,4855$

The slope $\mathrm{B}$ is $-0.4855(\mathrm{~B}=-\lambda)$ and $\lambda=0.4855$ represents the decay constant from which it is possible to obtain a mean lifetime for muons of :

$\tau=(1 / \lambda)=2,06 \mu \mathrm{s}$

The value of $\mathrm{N}_{\mathrm{o}}$ accounts for the background $\left(\ln \left(\mathrm{N}_{\mathrm{o}}\right)\right.$. This background term (which is present throughout the counting process) is best observable at the tail of the exponential.

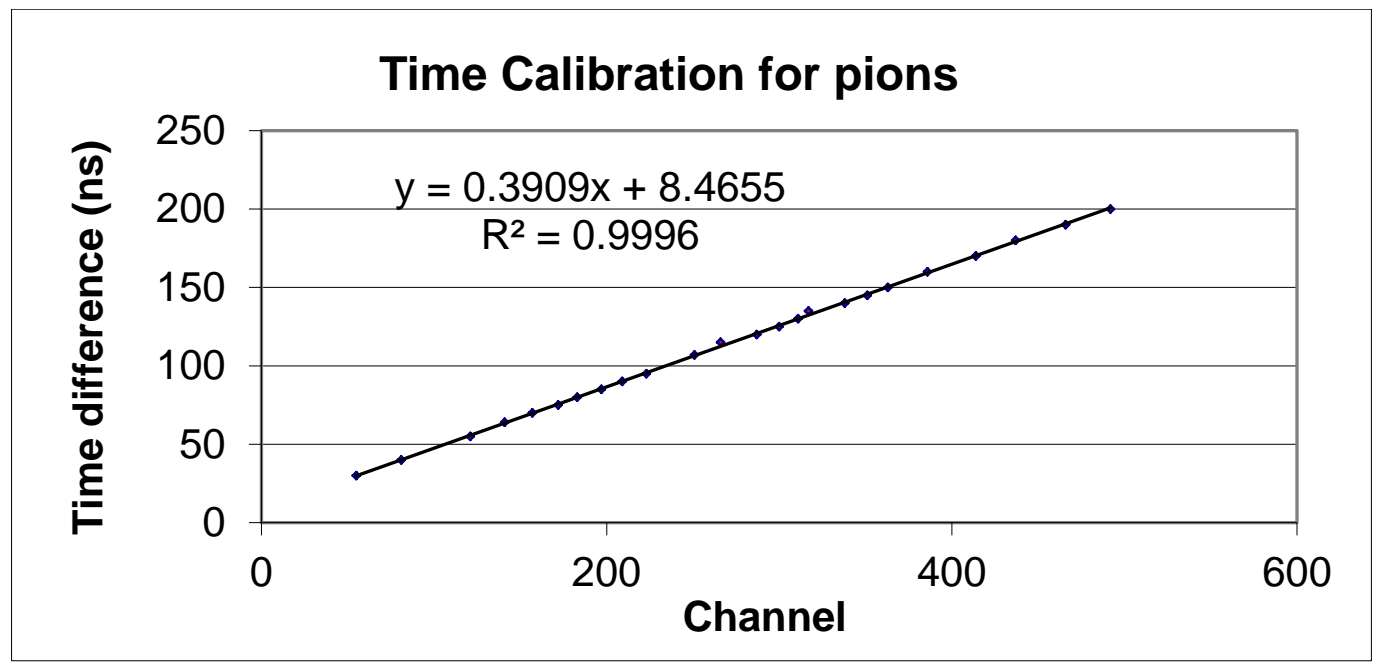

Fig.35. The time calibration for pion counting

It is possible to observe that the relation between the channel number and the time difference is also linear.

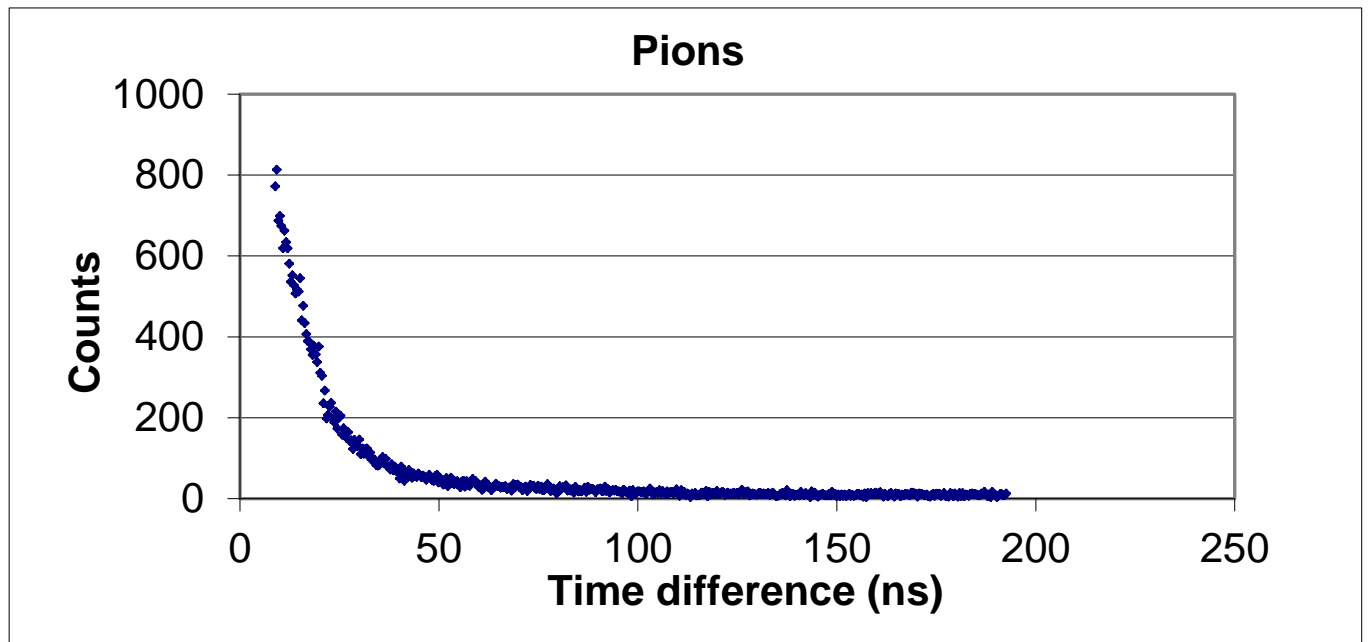

Fig.36. The spectrum obtained for the pion decay events 
Because the data taken (see figure 33) only reproduce a part of the exponential where the tail is not easily seen, the fit didn't include this term. Nevertheless, this background is normally considered to be small and besides, it could be controlled by the threshold selection at the discriminator during the set-up of the experiment [35]. In the same way, the results for the pions are shown in the figures 35,36 and 37.

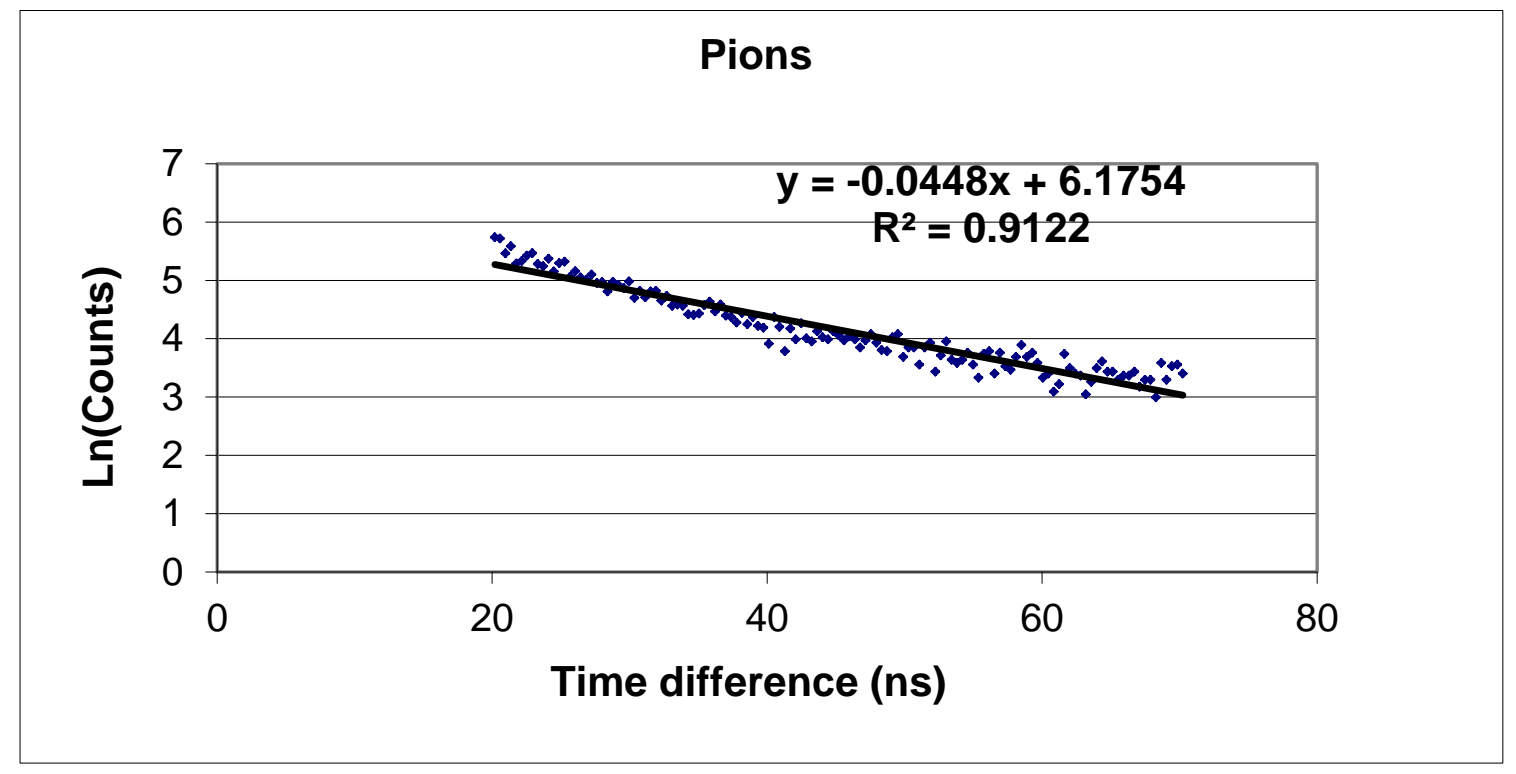

Fig.37. The natural logarithm of the counts vs. the time difference for pions

$\mathrm{N}=\mathrm{N}_{\mathrm{o}} \mathrm{e}^{-\lambda \mathrm{t}} \quad \ln (\mathrm{N})=\ln \left(\mathrm{N}_{\mathrm{o}}\right)-\lambda \mathrm{t} \quad \mathrm{y}=\mathrm{A}+\mathrm{Bt} \quad \mathrm{B}=-\lambda \quad \mathrm{y}=\ln (\mathrm{N}) \quad \mathrm{A}=\operatorname{Ln}\left(\mathrm{N}_{\mathrm{o}}\right)$

For pions, the best line $\mathrm{y}=\mathrm{A}+\mathrm{Bt}$ fitted to the data points is:

$\mathrm{y}=6.1754-0.0448 \mathrm{t} \quad \ln \left(\mathrm{N}_{\mathrm{o}}\right)=6,1754 \quad \mathrm{~N}_{\mathrm{o}}=478,38 \quad \lambda=0,0448$

The slope $\mathrm{B}$ is $-0.0448(\mathrm{~B}=-\lambda)$ and $\lambda=0.048$ represents the decay constant from which we can obtain a mean lifetime for pions of:

$\tau=(1 / \lambda)=22.32 \mathrm{~ns}$.

\subsection{Uncertainties of the results}

The errors at the measurements of a counting experiment like in our case are basically of a Poissonian type: events that occur at random but at a definite average rate. Thus, the error in the count is just the standard deviation of a Poisson distribution. It is given by the formula: $\sigma=\sqrt{\mu}=\sqrt{\mathrm{v}}$, where $\mu$ is the mean of a Poisson distribution which is equal to $\mathrm{v}$, the number of the counts in a counting experiment [35].

After plotting the data points and taking the natural logarithm of the counts and plotting again, a linear fit is obtained. The relationship that follows is:

$\mathrm{N}=\mathrm{N}_{\mathrm{o}} \mathrm{e}^{-\lambda t} \quad \ln (\mathrm{N})=\ln \left(\mathrm{N}_{\mathrm{o}}\right)-\lambda \mathrm{t} \quad \mathrm{y}=\mathrm{A}+\mathrm{Bt} \quad \mathrm{y}=\ln (\mathrm{N}) \quad \mathrm{A}=\operatorname{Ln}\left(\mathrm{N}_{\mathrm{o}}\right) \quad \mathrm{B}=-\lambda$

Where, $\mathrm{N}$ is the number of counts: $\mathrm{y}=\ln$ (counts), $\mathrm{t}$ is the time difference, $\mathrm{A}$ and $\mathrm{B}$ are constants and $|\mathrm{B}|$ is the constant decay $\lambda$. The line fitted to the data is obtained via the least square's method. The square of the correlation 
coefficient $\left(\mathrm{R}^{2}\right)$ is very close to one both for muons and pions (see figures 34 and 37). It confirms us that the relation between Ln (counts) and time is linear as it was expected. Now, if we want to calculate the error in the mean lifetime, it is necessary to apply the method of least square. Because the errors of the Y coordinate ( $\mathrm{Ln}$ (Counts)) are not the same for all measurements, the method of the least squares takes the name of weighted least squares and a slight modification to the usual method is required [35]. First of all, the probability of obtaining a set of measurements $\mathrm{y}_{1}, \mathrm{y}_{2}, \ldots \mathrm{y}_{\mathrm{n}}$ is given by:

$\mathrm{P}_{\mathrm{A}, \mathrm{B}}\left(\mathrm{y}_{1}, \mathrm{y}_{2}, \ldots, \mathrm{y}_{\mathrm{n}}\right) \propto e^{-\frac{\chi^{2}}{2}}$

Where $\chi^{2}=\Sigma w_{i}\left(y_{i}-A-B t_{i}\right)^{2}$ and the term $w_{i}=\frac{1}{\sigma_{i}{ }^{2}}$ is the weighting factor which gives the name to the method. The $\sigma_{\mathrm{i}}{ }^{2}$ are the errors for the counts, which in this case are not equals. When the quantity $\chi^{2}$ (chi square) is minimized, (it is differentiated this quantity with respect to A and B and made it equal to zero), the constants $\mathrm{A}$ and B can be found:

$\mathrm{A}=\left(\mathrm{M}_{22} \mathrm{~V}_{1}-\mathrm{M}_{12} \mathrm{~V}_{2}\right) / \Delta, \mathrm{B}=\left(\mathrm{M}_{11} \mathrm{~V}_{2}-\mathrm{M}_{21} \mathrm{~V}_{1}\right) / \Delta, \Delta=\mathrm{M}_{11} \mathrm{M}_{22}-\mathrm{M}_{12} \mathrm{M}_{21}$

Where,: $M_{11}=\Sigma \frac{1}{\sigma_{i}{ }^{2}} \quad M_{12}=\Sigma \frac{t_{i}}{\sigma_{i}{ }^{2}} \quad M_{21}=\Sigma \frac{t_{i}}{\sigma_{i}} \quad M_{22}=\Sigma \frac{t_{i}{ }^{2}}{\sigma_{i}{ }^{2}}$

$V_{1}=\Sigma \frac{y_{i}}{\sigma_{i}^{2}} \quad V_{2}=\Sigma \frac{t_{i} y_{i}}{\sigma_{i}^{2}}$

The values of $y_{i}, t_{i}$, and the uncertainties of each measurement $\sigma_{i}$ are given by the respective data measurements and theirs uncertainties.

The uncertainties in A and B are given by:

$\sigma_{A}^{2}=\frac{M_{22}}{\Delta} \quad \sigma_{B}^{2}=\frac{M_{11}}{\Delta}$

From these last two formulas, the uncertainty at the mean lifetime can be found. The mean life time is given by:

$|\mathrm{B}|=\lambda \quad \tau=1 / \lambda \quad \tau=1 /|\mathrm{B}|$ and according to the uncertainty propagation theory: $\sigma_{\tau}=\left|\frac{\partial \tau}{\partial B}\right| \sigma_{B}=\frac{\sigma_{B}}{B^{2}}$

The results for muons and pions are:

Pions: $\tau(\mathrm{ns}) \pm \Delta \tau(\mathrm{ns})=22.32 \pm 0.29$ (ns)

Muons: $\tau(\mu \mathrm{s}) \pm \Delta \tau(\mu \mathrm{s})=2.06 \pm 0.06(\mu \mathrm{s})$

The best experimental value for the mean lifetime for pions is $25,5 \mathrm{~ns}$ and for muons is 2,2 us. It is obtained a best experimental result for the mean lifetime of the muons. Nevertheless, the mean lifetime for the pion is not very far to the best experimental value of $25,5 \mathrm{~ns}$.

\subsection{Relativistic Energy Momentum Relation}

The relativistic energy formula for a particle is given by:

$\mathrm{E}^{2}=\mathrm{p}^{2} \mathrm{c}^{2}+\mathrm{m}_{\mathrm{o}}{ }^{2} \mathrm{c}^{4}$

Which it is reduced to $\mathrm{E}=\mathrm{pc}$ for photons. 
The quantity pc is proportional to the relativistic momentum but it has energy unit [35]. It is useful in many high energy calculations by using the next relationships:

$\mathrm{p}^{2} \mathrm{c}^{2}=\mathrm{E}^{2}-\mathrm{m}_{\mathrm{o}}^{2} \mathrm{c}^{4}$

$\mathrm{E}=\mathrm{mc} \mathrm{c}^{2}=\mathrm{K}+\mathrm{m}_{0} \mathrm{c}^{2}$

Where: $m=\gamma m_{0} \quad E=\gamma m_{0} c^{2}$

$\gamma=1 / \sqrt{1-\beta^{2}}$ and $\beta=\mathrm{v} / \mathrm{c}$

It is replaced $\mathrm{E}=\mathrm{K}+\mathrm{m}_{\mathrm{o}} \mathrm{c}^{2}$ in $\mathrm{p}^{2} \mathrm{c}^{2}=\mathrm{E}^{2}-\mathrm{m}_{\mathrm{o}}{ }^{2} \mathrm{c}^{4}$ and it is obtained:

$\mathrm{pc}=\sqrt{\mathrm{K}\left(\mathrm{K}+2 \mathrm{~m}_{\mathrm{o}} \mathrm{c}^{2}\right)}$

For the most ordinary processes, the kinetic energy is much less than the energy of the rest mass [35]. Thus, the first term can usually be neglected. This situation leads to the useful approximation:

$\mathrm{pc}=\sqrt{2 \mathrm{Km}_{\mathrm{o}} \mathrm{c}^{2}}$

For extreme relativistic velocities, where $\mathrm{E}$ is so much larger than $\mathrm{m}_{\mathrm{o}} \mathrm{c}^{2}$, then $\mathrm{pc}$ is approximately $\mathrm{E}$ :

$E^{2}=p^{2} c^{2}+m_{o}^{2} c^{4} E \gg>m_{0} c^{2} E \approx p c$.

5.7. The mean lifetime of muons at rest is $\tau=2.2 \mu s$ (muon framework), their rest mass is $m_{o}=207 m_{e}$.

What is the kinetic energy of a muon produced at an altitude of $20 \mathrm{~km}$

which reaches the sea level

Relatively:

$\mathrm{L}=\mathrm{L}_{\mathrm{o}} \sqrt{1-\beta^{2}} \mathrm{~L}=\mathrm{L}_{\mathrm{o}} / \gamma \quad$ (Length Contraction) $\quad \gamma=1 / \sqrt{1-\beta^{2}}$ and $\beta=\mathrm{v} / \mathrm{c}$

$\mathrm{L}_{0}$ : measured length at rest $\mathrm{L}_{0}=\mathrm{x}=20 \mathrm{~km}$

L: Measured length at relativistic velocities at the framework of the muons: $L=c \tau$

Then, $\mathrm{L}=\mathrm{c} \tau \quad \mathrm{L}_{\mathrm{o}}=\mathrm{x} \quad \mathrm{c} \tau=\mathrm{x} / \gamma \quad \mathrm{x}=\gamma \mathrm{c} \tau$

$\mathrm{x}=20 \mathrm{~km} \quad \gamma \mathrm{c} \tau=20 \mathrm{~km} \quad \mathrm{c}=300000 \mathrm{~km} / \mathrm{s} \quad \tau=2.2 \mu \mathrm{s}$

Thus, $\gamma=30.303$

The relativistic total energy is given:

$\mathrm{E}_{\mu}=\gamma \mathrm{m}_{\mu} \mathrm{c}^{2} \quad \gamma=1 / \sqrt{1-\beta^{2}}$ and $\beta=\mathrm{v} / \mathrm{c}$

Where, $\mathrm{m}_{\mu}=207 \mathrm{~m}_{\mathrm{e}}=207 *\left(0.511 \mathrm{MeV} / \mathrm{c}^{2}\right)=105.77 \mathrm{MeV} / \mathrm{c}^{2}$

Therefore, it is obtained: $\mathrm{E}_{\mu}=(30.303)(207)\left(0.511 \mathrm{MeV} / \mathrm{c}^{2}\right) \mathrm{c}^{2}=3205.36 \mathrm{MeV}$.

The kinetic energy of a muon produced at an altitude of $20 \mathrm{~km}$ which reaches the sea level is given by: $\mathrm{E}=\mathrm{K}+\mathrm{m}_{\mathrm{o}} \mathrm{c}^{2}$ $\mathrm{K}_{\mu}=\mathrm{E}_{\mu}-\mathrm{m}_{\mu} \mathrm{c}^{2}=3099.58 \mathrm{MeV} / \mathrm{c}^{2}$ 


\subsection{Determine the kinetic energy of muons from pion decay at rest}

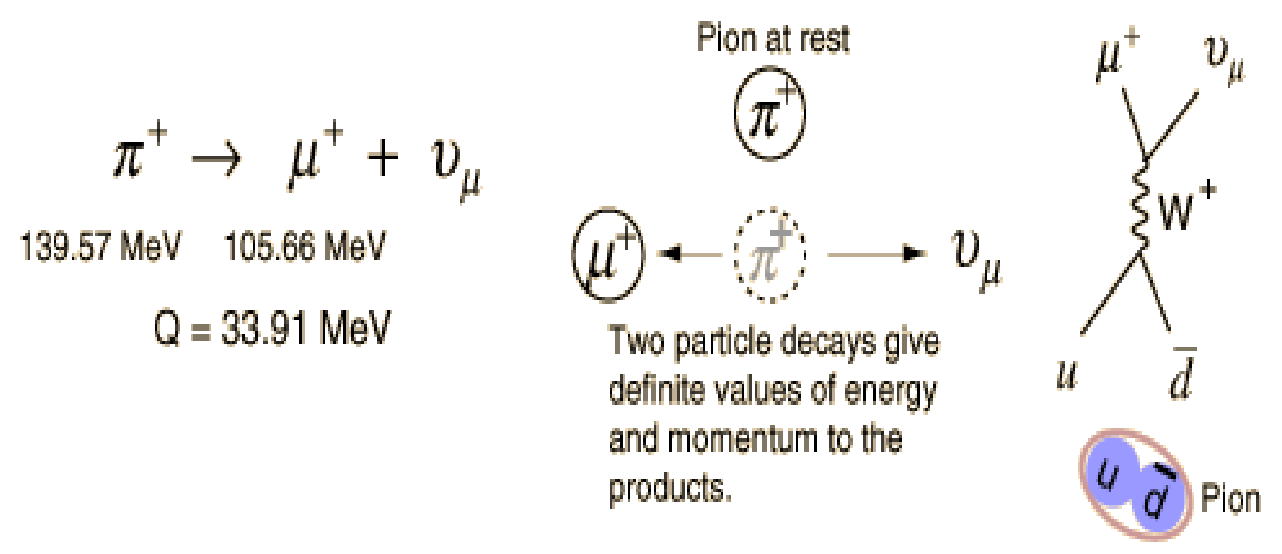

Fig.38. Pion decay at rest

For the neutrino product which has a negligible mass, it is applied the Dirac equation $E^{2}=m^{2} c^{4}+(p c)^{2}: \quad E_{v}=p c$

The muon must have the same value of $\mathrm{E}_{\mu}=\mathrm{pc}$ in the opposite direction to conserve the momentum. It is possible to observe by applying the equation to the muon $E^{2}=\left(K+m c^{2}\right)^{2}$ and $E^{2}=m^{2} c^{4}+(p c)^{2}$, that $K^{2}+2 K m c^{2}=(p c)^{2}$ and so $(\mathrm{pc})_{v}=(\mathrm{pc})_{\mu}$ and $(\mathrm{pc})_{v}{ }^{2}=(\mathrm{pc})_{\mu}{ }^{2}$

$(\mathrm{pc})^{2}=\left(\mathrm{KE}_{\mu}\right)^{2}+2 \mathrm{KE}_{\mu} \mathrm{m}_{\mu} \mathrm{c}^{2}$ where $\mathrm{Q}=\mathrm{KE}_{\mu}+\mathrm{pc} \quad \mathrm{KE}_{\mu}=\mathrm{Q}-\mathrm{pc}$

Expanding in terms of Q and pc, we obtain:

$(\mathrm{pc})^{2}=\mathrm{Q}^{2}-2 \mathrm{Qpc}+(\mathrm{pc})^{2}+2(\mathrm{Q}-\mathrm{pc}) \mathrm{m}_{\mu} \mathrm{c}^{2}$

$p c=\frac{Q^{2}+2 Q m_{\mu} c^{2}}{2\left(Q+m_{\mu} c^{2}\right)}$

By replacing the values of: $\mathrm{Q}=33.91 \mathrm{MeV}, \mathrm{m}_{\mu}=105.66 \mathrm{MeV} / \mathrm{c}^{2}$

$\mathrm{pc}=29.79 \mathrm{MeV}$

$\mathrm{KE}_{\mu}=\mathrm{Q}-\mathrm{pc}=33.91-29.79=4.12 \mathrm{MeV}$

This decay is identified as two particle decay. The measurement of the kinetic energy of the muon (4.12 MeV) shows that the most of the energy is carried by the neutrino. Also, it is consistent with the zero mass for the neutrino, since the kinetic energy of the muon was calculated with the assumption of zero mass for the neutrino.

The small bump at $70 \mathrm{MeV}$ in the energy spectrum of electron from muon decay at rest can be explained considering another possible mechanism of pion decay. It is a pion decaying directly into an electron and an antineutrino.

$\pi^{-} \rightarrow \mu^{-}+\overline{v_{\mu}}$

$\mu^{-} \rightarrow e^{-}+\overline{v_{e}}+v_{\mu}$

$\pi^{-} \rightarrow e^{-}+\overline{v_{e}}+v_{\mu}+\overline{v_{\mu}}$ 
$\pi^{-} \rightarrow e^{-}+\overline{v_{e}} \quad$ (other possible mechanism of pion decay)

By taking the energy and the momentum conservation for this reaction, we have:

$\mathrm{E}_{\pi-}=\mathrm{E}_{\mathrm{e}-\mathrm{E}}+\mathrm{E} \overline{v_{e}} \quad \mathrm{E}^{2}=\mathrm{m}^{2} \mathrm{c}^{4}+(\mathrm{pc})^{2}$ pion at rest: $\mathrm{E}_{\pi-}=\mathrm{m}_{\pi} \mathrm{c}^{2} \quad \mathrm{E}_{\mathrm{e}}=\left(\mathrm{p}_{\mathrm{e}}{ }^{2} \mathrm{c}^{2}+\mathrm{m}_{\mathrm{e}}{ }^{2} \mathrm{c}^{4}\right)^{1 / 2}$

For the neutrino product which has a negligible mass, it is applied the Dirac equation $E^{2}=m^{2} c^{4}+(p c)^{2}: E_{v}=p c$

$\mathrm{m}_{\pi} \mathrm{c}^{2}=\left(\mathrm{p}_{\mathrm{e}}{ }^{2} \mathrm{c}^{2}+\mathrm{m}_{\mathrm{e}}{ }^{2} \mathrm{c}^{4}\right)^{1 / 2}+\mathrm{p}_{\mathrm{e}} \mathrm{c}$

$\mathrm{m}_{\pi} \mathrm{c}^{2}-\mathrm{p}_{\mathrm{e}} \mathrm{c}=\left(\mathrm{p}_{\mathrm{e}} \mathrm{c}^{2}+\mathrm{m}_{\mathrm{e}}{ }^{2} \mathrm{c}^{4}\right)^{1 / 2}$

$\mathrm{m}_{\pi}{ }^{2} \mathrm{c}^{4}-2 \mathrm{~m}_{\pi} \mathrm{c}^{2} \mathrm{p}_{\mathrm{e}} \mathrm{c}+\mathrm{p}_{\mathrm{e}}{ }^{2} \mathrm{c}^{2}=\mathrm{p}_{\mathrm{e}}{ }^{2} \mathrm{c}^{2}+\mathrm{m}_{\mathrm{e}}{ }^{2} \mathrm{c}^{4}$

$p_{e} c=\frac{1}{2} m_{\pi} c^{2}-\frac{1}{2} \frac{m_{e}^{2} c^{4}}{m_{\pi} c^{2}}$

By neglecting the term of the right of the last expression $m_{e}{ }^{2} c^{4} \gg m_{\pi} c^{2}$, it is obtained:

$p_{e} c=\frac{1}{2} m_{\pi} c^{2} \quad \mathrm{~m}_{\pi}=139,56 \mathrm{MeV} / \mathrm{c}^{2}$

Therefore, it is obtained: $\mathrm{p}_{\mathrm{e}} \mathrm{c}=70 \mathrm{MeV}$ which is just the value of the energy bump seen at the electron energy spectrum. It is a small bump because this reaction has other competing reactions.

\section{The Variant Mass for a particle in a Gravitational Field}

\subsection{A particle orbiting an almost static massive planet or Sun}

The mass of the particle is $\mathrm{m}$ and the mass of the massive planet or Sun is M. The particle moves around the planet in a circular motion with radius R. If the planet moves around the Sun, the exact motion is an ellipse as Kepler has established [33].

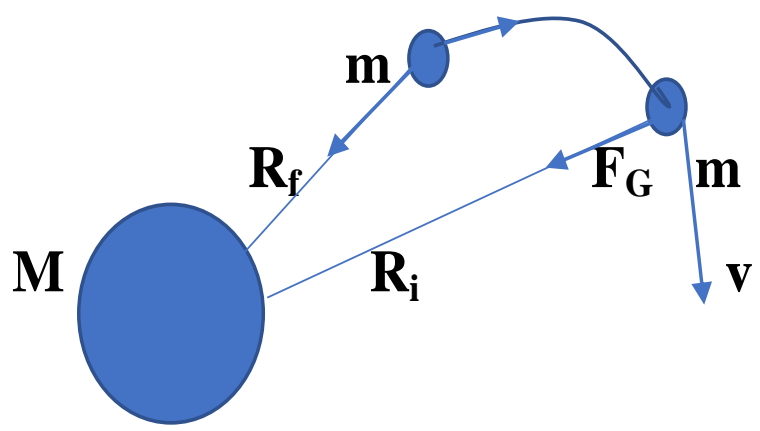

Fig.39. A particle orbiting an almost massive Planet or Sun due the Gravitational Force

The gravitational force between the particle and the planet gives out the Gravitational Potential Energy. Besides, the particle emits gravitational energy due the presence of a massive planet near the particle. Then, the mass of the particle decreases due the emission of the gravitational energy. As result of it, the particle changes a little its trajectory by decreasing a little the distance $\mathrm{R}$ with the planet. Then, the particle starts to move in a circular or elliptical motion (rotational motion) around the massive planet due the initial velocity of the particle. It is the case of the planetary system moving or orbiting around its massive star. The effect of the little change of the trajectory is possible to measure at the motion of Mercury (Perihelion Precession) [33]. 


\subsection{Formula development of the total mass and energy of the particle in a gravitational field}

$c^{2} d m=\frac{d p}{d t} d s+\frac{G M m}{r^{2}} d r$

$c^{2} d m=\frac{d(m v)}{d t} d s+\frac{G M m}{r^{2}} d r$

$c^{2} d m=m v d v+v^{2} d m+\frac{G M m}{r^{2}} d r$

$c^{2} d m-v^{2} d m=m v d v+\frac{G M m}{r^{2}} d r$

$\frac{G M m}{r^{2}}=m \frac{v^{2}}{r}$

$\frac{G M}{r}=v^{2}$

$-\frac{G M}{2 r^{2}} d r=v d v$

$c^{2} d m-\frac{G M}{r} d m=-\frac{G M m}{2 r^{2}} d r+\frac{G M m}{r^{2}} d r$

$\left(c^{2}-\frac{G M}{r}\right) d m=\frac{G M m}{2 r^{2}} d r$

$\frac{d m}{m}=\frac{\frac{G M}{2 r^{2}}}{\left(c^{2}-\frac{G M}{r}\right)} d r$

$\ln \left(\frac{m}{m_{o}}\right)=\frac{1}{2} \ln \left(\frac{c^{2}-\frac{G M}{r}}{c^{2}-\frac{G M}{r_{o}}}\right) \quad \mathrm{m}=\mathrm{m}_{\mathrm{o}} \quad \mathrm{r}_{\mathrm{o}}=\infty$

$m=m_{o} \sqrt{\left(1-\frac{G M}{r c^{2}}\right)} \quad \frac{G M}{r}=v^{2}$

$m=m_{o} \sqrt{\left(1-\frac{v^{2}}{c^{2}}\right)}$ mass for a particle in a gravitational field

$\Delta \mathrm{m}=\mathrm{m}_{\mathrm{o}}-\mathrm{m}$

$\Delta \mathrm{m}=\mathrm{m}_{\mathrm{o}}\left(1-\sqrt{\left(1-\frac{\mathrm{GM}}{\mathrm{rc}^{2}}\right)}\right.$ total lost mass of the particle

$\mathrm{GM} / \mathrm{r}<<\mathrm{c}^{2}$

$m=m_{o}\left(1-\frac{G M}{2 r c^{2}}\right)$

$m c^{2}=m_{o} c^{2}-\frac{G M m_{o}}{2 r}$

$\Delta \mathrm{mc}^{2}=\mathrm{m}_{\mathrm{o}} \mathrm{c}^{2}-\mathrm{mc}^{2}=\left(\frac{\mathrm{GMm}_{\mathrm{o}}}{2 \mathrm{R}}\right)$ as the total mass decreasing of the particle in a gravitational field for the classic approach where $\mathrm{r}=\mathrm{R}$

The formula for the mass given by the Relativity Theory is as follows: 
$m=m_{o} \frac{\sqrt{1-2 \frac{G M}{r c^{2}}}}{\sqrt{1-\frac{G M}{r c^{2}}}}$

$\mathrm{GM} / \mathrm{r}<<\mathrm{c}^{2}$

$\sqrt{1-2 \frac{G M}{r c^{2}}} \approx 1-\frac{G M}{r c^{2}}$

$\left(1-\frac{G M}{r c^{2}}\right)^{-1 / 2} \approx 1+\frac{G M}{2 r c^{2}}$

$m=m_{o}\left(1-\frac{G M}{2 r c^{2}}\right) \quad$ (as the classic approach).

$m c^{2}=m_{o} c^{2}+E \quad \mathrm{E}=m c^{2}-m_{o} c^{2}$

$\mathrm{E}=-\frac{G M m_{0}}{2 r} \quad$ (as the classic approach).

The next figure shows the plot of $\Delta \mathrm{m} / \mathrm{m}_{\mathrm{o}}$ versus the radius $\mathrm{r}(\mathrm{m})$ from the mass development formula (green) and for the mass formula from General Relativity (blue) where $M$ is the Sun Mass and $r$ is the distance from the Massive object with mass $m_{o}$ or Planet to the Sun and $\Delta \mathrm{m}=\mathrm{m}_{\mathrm{o}}-\mathrm{m}$.

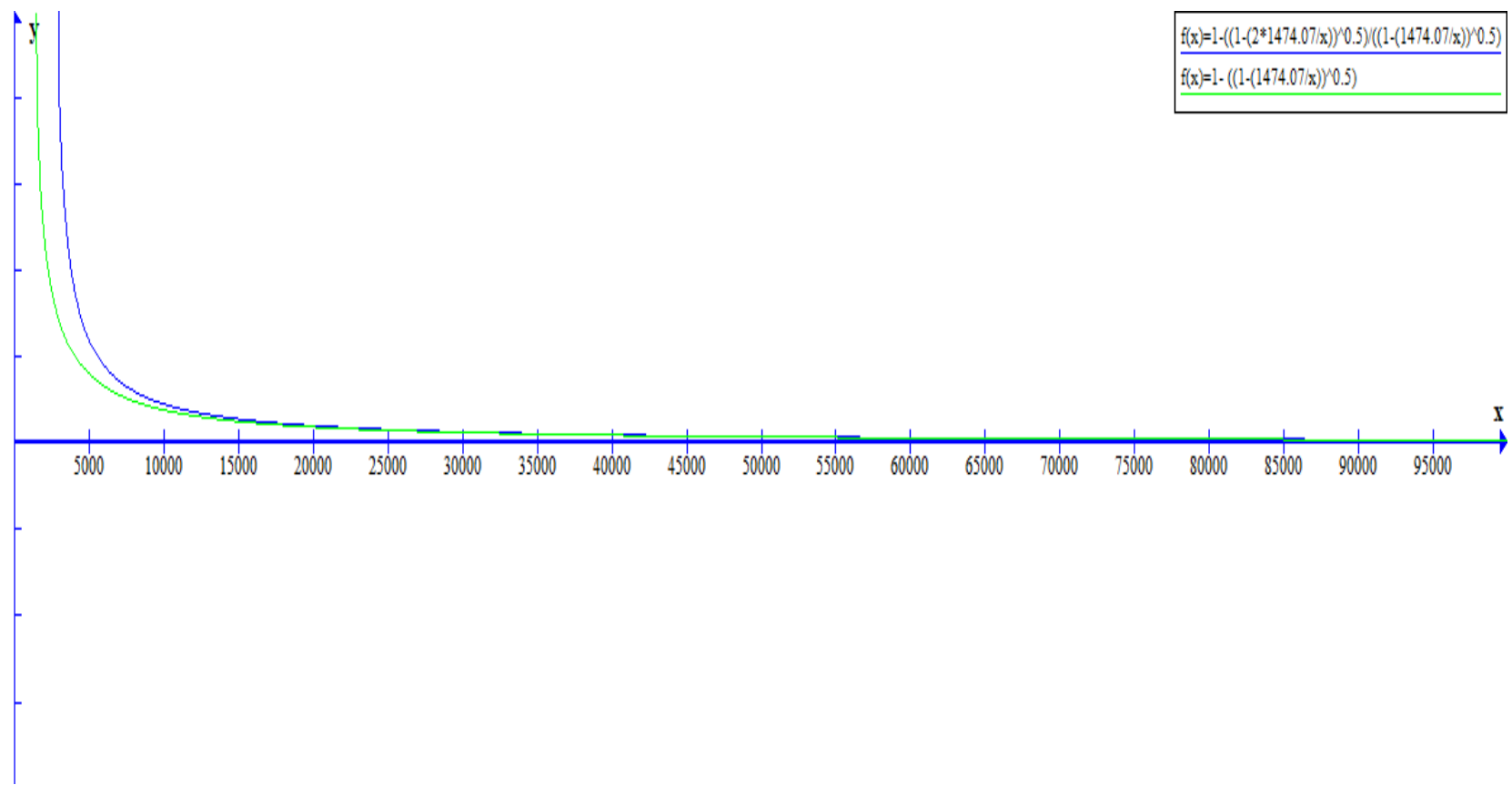

Fig.40. $\Delta \mathrm{m} / \mathrm{m}_{\mathrm{o}}$ versus the radius $\mathrm{r}(\mathrm{m})$ from the mass development formula (green) and for the mass formula from General Relativity (blue) where $\mathrm{M}$ is the Sun Mass and $\mathrm{r}$ is the distance from the Massive object with mass $\mathrm{m}_{\mathrm{o}}$ or Planet to the Sun

$\Delta \mathrm{m} / \mathrm{m}_{\mathrm{o}}=\left(1-\sqrt{\left(1-\frac{\mathrm{GM}}{\mathrm{rc}^{2}}\right)} \quad\right.$ Development mass formula

$\Delta \mathrm{m} / \mathrm{m}_{\mathrm{o}}=\left(1-\frac{\sqrt{1-2 \frac{\mathrm{GM}}{\mathrm{rc}^{2}}}}{\sqrt{1-\frac{\mathrm{GM}}{\mathrm{rc}^{2}}}}\right) \quad$ General Relativity formula 
Both formulas for the decrease mass of the particle agree at distances very far from the massive object and also at distances very near to the massive object with high accuracy. Then, both models: the Newton Approach with a gravitational field interaction and the Relativity Theory with a curvature of the space time are in agreement at the results for the total mass decreasing of the particle in a gravitational field [33].

For the total energy of the particle, it is obtained:

$\mathrm{E}=m c^{2}-m_{o} c^{2}$

$E=m_{o} c^{2}\left(\sqrt{\left(1-\frac{G M}{r c^{2}}\right.}-1\right)$ energy for a particle in a gravitational field

$\mathrm{GM} / \mathrm{r}<<\mathrm{c}^{2}$

$\mathrm{E}=-\frac{G M m_{0}}{2 r} \quad($ as the classic approach $)$

The total mass energy lost is given by the formula demonstrated and it is equal in absolute value to the total bond energy of the system which is emitted as gravitational energy when the particle goes from one orbit to another orbit with less radius. The emission of the total gravitational energy E produces a lost mass of the particle $\mathrm{m}=\mathrm{E} / \mathrm{c}^{2}$.

If $\mathrm{R}_{\mathrm{f}}<\mathrm{R}_{\mathrm{i}} \Delta E=E_{f}-E_{i}=\left(-\frac{G M m}{2 R_{f}}\right)-\left(-\frac{G M m}{2 R_{i}}\right)$ is negative, it is given out energy which means that the particle losses mass, the particle increases the kinetic energy and the velocity but decreases the gravitational potential energy (more negative). Part of the losses energy (gravitational energy emission) is given out by decreasing the potential energy and part by increasing the kinetic energy [33]. If $R_{f}>R_{i} \Delta E$ is positive, which means that additional energy is given to the particle or additional work is done on the system, the particle decreases the kinetic energy and the velocity but increases the potential energy (less negative). Part of the work or additional energy is used to increase the potential energy and part to diminish the kinetic energy [33].

\section{Binary Stars: The Physics and emission of the gravitational energy and the Variant Mass}

If the particle and the planet have comparable and similar masses, then they start to move around each other and orbiting around their common barycenter (rotational motion) [33].

\subsection{Case $m_{1} \neq m_{2}$}

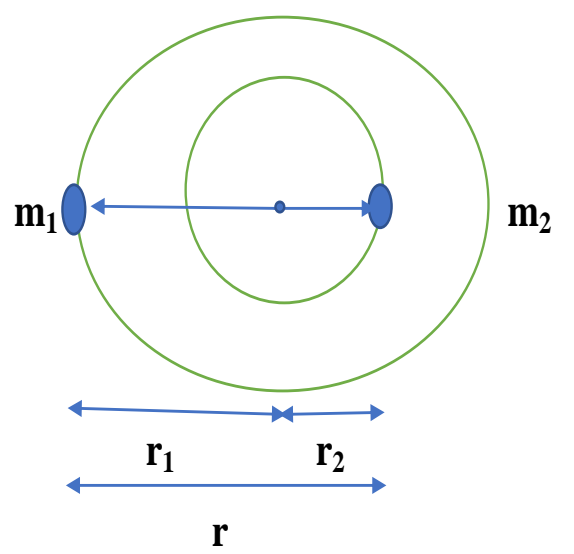

Fig.41. Binary Stars orbiting around their common barycenter due the Gravitational Force 
It occurs in binary stars for example.

Also, they emit gravitational energy and as a consequence, there is a decrease in their masses and the distance between them due this energy emission [33].

By considering the center of mass in $r=0$, it is obtained:

$\mathrm{m}_{1} \mathrm{r}_{1}=\mathrm{m}_{2} \mathrm{r}_{2} \quad \mathrm{r}_{1}=\mathrm{m}_{2} \mathrm{r}_{2} / \mathrm{m}_{1}$

$\mathrm{r}=\mathrm{r}_{1}+\mathrm{r}_{2}$

$\mathrm{r}=\left(\mathrm{m}_{2} \mathrm{r}_{2} / \mathrm{m}_{1}\right)+\mathrm{r}_{2} \quad \mathrm{r}=\left(\mathrm{m}_{1}+\mathrm{m}_{2}\right) \mathrm{r}_{2} / \mathrm{m}_{1}$

$\mathbf{r}_{2}=\left(\mathbf{m}_{1}\right) \mathbf{r} /\left(\mathbf{m}_{1}+\mathbf{m}_{2}\right)$

$\mathrm{r}_{2}=\mathrm{m}_{1} \mathrm{r}_{1} / \mathrm{m}_{2}$

$\mathrm{r}=\mathrm{r}_{1}+\mathrm{r}_{2} \quad \mathrm{r}=\mathrm{r}_{1}+\mathrm{m}_{1} \mathrm{r}_{1} / \mathrm{m}_{2}$

$\mathrm{r}=\left(\mathrm{m}_{1}+\mathrm{m}_{2}\right) \mathrm{r}_{1} / \mathrm{m}_{2}$

$\mathbf{r}_{1}=\left(\mathbf{m}_{2}\right) \mathbf{r} /\left(\mathbf{m}_{1}+\mathbf{m}_{2}\right)$

$\mathrm{K}_{1}=\frac{1}{2} \mathrm{~m}_{1} \mathrm{v}_{1}^{2} \quad \mathrm{v}_{1}=\mathrm{w}_{1} \mathrm{r}_{1} \quad \mathrm{w}_{1}=\mathrm{v}_{1} / \mathrm{r}_{1}$

$\mathrm{G} \frac{\mathrm{m}_{1} \mathrm{~m}_{2}}{\mathrm{r}^{2}}=\mathrm{m}_{1} \frac{\mathrm{v}_{1}^{2}}{\mathrm{r}_{1}}$

$G \frac{m_{2} r_{1}}{r^{2}}=v_{1}{ }^{2} \quad G \frac{m_{2}{ }^{2}}{r\left(m_{1}+m_{2}\right)}=v_{1}{ }^{2}$

$\mathrm{w}_{1}^{2}=\frac{\mathrm{v}_{1}^{2}}{\mathrm{r}_{1}{ }^{2}}=\mathrm{G} \frac{\mathrm{m}_{2}}{\mathrm{r}^{2} \mathrm{r}_{1}} \quad \mathrm{w}_{1}{ }^{2}=\mathrm{G} \frac{\left(\mathrm{m}_{1}+\mathrm{m}_{2}\right)}{\mathrm{r}^{3}}$

$\mathrm{K}_{1}=\frac{1}{2} \mathrm{~m}_{1} \mathrm{v}_{1}^{2}=\frac{1}{2} \mathrm{~m}_{1} \mathrm{G} \frac{\mathrm{m}_{2} \mathrm{r}_{1}}{\mathrm{r}^{2}}=\frac{1}{2} \mathrm{G} \frac{\mathrm{m}_{1} \mathrm{~m}_{2} \mathrm{r}_{1}}{\mathrm{r}^{2}}$

$\mathrm{r}_{1}=\left(\mathrm{m}_{2}\right) \mathrm{r} /\left(\mathrm{m}_{1}+\mathrm{m}_{2}\right)$

$K_{1}=\frac{1}{2} G \frac{m_{1} m_{2}}{r} \frac{m_{2}}{\left(m_{1}+m_{2}\right)}$ kinetic energy for $m_{1}$

$\mathrm{K}_{2}=\frac{1}{2} \mathrm{~m}_{2} \mathrm{v}_{2}{ }^{2} \quad \mathrm{v}_{2}=\mathrm{w}_{2} \mathrm{r}_{2} \quad \mathrm{w}_{2}=\mathrm{v}_{2} / \mathrm{r}_{2}$

$\mathrm{G} \frac{\mathrm{m}_{1} \mathrm{~m}_{2}}{\mathrm{r}^{2}}=\mathrm{m}_{2} \frac{\mathrm{v}_{2}^{2}}{\mathrm{r}_{2}}$

$\mathrm{G} \frac{\mathrm{m}_{1} \mathrm{r}_{2}}{\mathrm{r}^{2}}=\mathrm{v}_{2}{ }^{2} \quad \mathrm{G} \frac{\mathrm{m}_{1}{ }^{2}}{\mathrm{r}\left(\mathrm{m}_{1}+\mathrm{m}_{2}\right)}=\mathrm{v}_{2}{ }^{2}$

$\mathrm{w}_{2}{ }^{2}=\frac{\mathrm{v}_{2}{ }^{2}}{\mathrm{r}_{2}{ }^{2}}=\mathrm{G} \frac{\mathrm{m}_{1}}{\mathrm{r}^{2} \mathrm{r}_{2}} \quad \mathrm{w}_{2}{ }^{2}=\mathrm{G} \frac{\left(\mathrm{m}_{1}+\mathrm{m}_{2}\right)}{\mathrm{r}^{3}}$

$\mathrm{K}_{2}=\frac{1}{2} \mathrm{~m}_{2} \mathrm{v}_{2}{ }^{2}=\frac{1}{2} \mathrm{~m}_{2} \mathrm{G} \frac{\mathrm{m}_{1} \mathrm{r}_{2}}{\mathrm{r}^{2}}=\frac{1}{2} \mathrm{G} \frac{\mathrm{m}_{1} \mathrm{~m}_{2} \mathrm{r}_{2}}{\mathrm{r}^{2}}$

$\mathrm{r}_{2}=\left(\mathrm{m}_{1}\right) \mathrm{r} /\left(\mathrm{m}_{1}+\mathrm{m}_{2}\right)$ 
$K_{2}=\frac{1}{2} G \frac{m_{1} m_{2}}{r} \frac{m_{1}}{\left(m_{1}+m_{2}\right)}$ kinetic energy for $m_{2}$

Therefore, $\mathrm{m}_{1}$ and $\mathrm{m}_{2}$ have different kinetic energies $\mathrm{K}$.

$\mathrm{K}_{\mathrm{T}}=\mathrm{K}_{1}+\mathrm{K}_{2}=\frac{1}{2} \mathrm{G} \frac{\mathrm{m}_{1} \mathrm{~m}_{2}}{\mathrm{r}} \frac{\mathrm{m}_{2}}{\left(\mathrm{~m}_{1}+\mathrm{m}_{2}\right)}+\frac{1}{2} \mathrm{G} \frac{\mathrm{m}_{1} \mathrm{~m}_{2}}{\mathrm{r}} \frac{\mathrm{m}_{1}}{\left(\mathrm{~m}_{1}+\mathrm{m}_{2}\right)}$

$K_{T}=\frac{1}{2} G \frac{m_{1} m_{2}}{r}$

$\mathbf{U}=-\mathbf{G} \frac{\mathbf{m}_{1} \mathbf{m}_{2}}{\mathbf{r}}$

Therefore, the total mechanical energy of the system of the binary stars which is given as emission of gravitational energy is:

$E=K_{T}+U=\frac{1}{2} G \frac{m_{1} m_{2}}{r}-G \frac{m_{1} m_{2}}{r}$

$\mathbf{E}=-\mathbf{G} \frac{\mathbf{m}_{1} \mathbf{m}_{2}}{2 \mathbf{r}}$

It is the same total energy formula of a particle orbiting an almost static massive object. Each mass $\mathrm{m}$ has equal angular velocity $w$, equal angular frequency $\mathrm{f}(\mathrm{w}=2 \pi \mathrm{f})$ and different kinetic energy with different linear velocities

v. The mass of each star is as follows:

$m_{1}=m_{01} \sqrt{\left(1-\frac{v_{1}^{2}}{c^{2}}\right)} \quad G \frac{m_{02}{ }^{2}}{r\left(m_{01}+m_{02}\right)}=v_{1}^{2}$

$m_{2}=m_{02} \sqrt{\left(1-\frac{v_{2}^{2}}{c^{2}}\right)} \quad G \frac{m_{01}{ }^{2}}{r\left(m_{01}+m_{02}\right)}=v_{2}^{2}$

Then, each star has different lost mass given by $\Delta \mathrm{m}_{1}=\mathrm{m}_{\mathrm{o} 1} \mathrm{c}^{2}-\mathrm{m}_{1} \mathrm{c}^{2}$ and $\Delta \mathrm{m}_{2}=\mathrm{m}_{\mathrm{o} 2} \mathrm{c}^{2}-\mathrm{m}_{2} \mathrm{c}^{2}$. Also, each star has different gravitational energy emission corresponding to the lost mass of the respective star [33]. It is possible to obtain the total energy at the classic approach:

$m_{1}=m_{o 1}\left(1-\frac{v_{1}^{2}}{2 c^{2}}\right) \quad \mathrm{E}_{1}=\mathrm{m}_{1} \mathrm{c}^{2}-\mathrm{m}_{\mathrm{o} 1} \mathrm{c}^{2}=-\frac{v_{1}{ }^{2}}{2} m_{o 1}$

$m_{2}=m_{o 2}\left(1-\frac{v_{2}{ }^{2}}{2 c^{2}}\right) \quad \mathrm{E}_{2}=\mathrm{m}_{2} \mathrm{c}^{2}-\mathrm{m}_{\mathrm{o} 2} \mathrm{c}^{2}=-\frac{v_{2}{ }^{2}}{2} m_{o 2}$

$\mathrm{E}=\mathrm{E}_{1}+\mathrm{E}_{2}=-\mathrm{G} \frac{\mathrm{m}_{02}{ }^{2}}{\mathrm{r}\left(\mathrm{m}_{\mathrm{o} 1}+\mathrm{m}_{\mathrm{o} 2}\right)} \frac{\mathrm{m}_{\mathrm{o} 1}}{2}-\mathrm{G} \frac{\mathrm{m}_{\mathrm{o1}}{ }^{2}}{\mathrm{r}\left(\mathrm{m}_{\mathrm{o} 1}+\mathrm{m}_{\mathrm{o} 2}\right)} \frac{\mathrm{m}_{\mathrm{o} 2}}{2}$

$\mathrm{E}=-\mathrm{G} \frac{\mathrm{m}_{\mathrm{0} 1} \mathrm{mo}_{2}}{2 \mathrm{r}}$ as the classic result

\subsection{Case $m_{1}=m_{2}=m$}

$\mathrm{mr}_{1}=\mathrm{mr}_{2} \quad \mathrm{r}_{1}=\mathrm{r}_{2}$

Each mass $m$ orbits the same circle of radius $r_{1}=r_{2}$

$\mathrm{r}=\mathrm{r}_{1}+\mathrm{r}_{2}$

$\mathrm{r}=2 \mathrm{r}_{1} \quad \mathrm{r}=2 \mathrm{r}_{2} \quad \mathrm{r}_{1}=\mathrm{r} / 2 \quad \mathrm{r}_{2}=\mathrm{r} / 2$ 
For $\mathrm{m}_{1}$, it is obtained:

$\mathrm{G} \frac{\mathrm{mm}}{\mathrm{r}^{2}}=\mathrm{m} \frac{\mathrm{v}_{1}{ }^{2}}{\mathrm{r}_{1}} \quad \mathrm{G} \frac{\mathrm{mr}_{1}}{\mathrm{r}^{2}}=\mathrm{v}_{1}{ }^{2} \quad \mathrm{G} \frac{\mathrm{m}}{2 \mathrm{r}}=\mathrm{v}_{1}{ }^{2} \quad \mathrm{r}_{1}=\mathrm{r} / 2$

$\mathrm{w}_{1}^{2}=\frac{\mathrm{v}_{1}^{2}}{\mathrm{r}_{1}^{2}}=\mathrm{G} \frac{\mathrm{m}}{\mathrm{r}^{2} \mathrm{r}_{1}}=\mathrm{G} \frac{2 \mathrm{~m}}{\mathrm{r}^{3}}$

$\mathrm{K}_{1}=\frac{1}{2} \mathrm{mv}_{1}^{2}$

$\mathrm{K}_{1}=\frac{1}{2} \mathrm{mv}_{1}^{2}=\frac{1}{2} \mathrm{mG} \frac{\mathrm{m}}{2 \mathrm{r}}=\mathrm{G} \frac{\mathrm{m}^{2}}{4 \mathrm{r}}$

For $\mathrm{m}_{2}$, it is obtained:

$\mathrm{G} \frac{\mathrm{mm}}{\mathrm{r}^{2}}=\mathrm{m} \frac{\mathrm{v}_{2}^{2}}{\mathrm{r}_{2}} \quad \mathrm{G} \frac{\mathrm{mr}_{2}}{\mathrm{r}^{2}}=\mathrm{v}_{2}{ }^{2} \quad \mathrm{G} \frac{\mathrm{m}}{2 \mathrm{r}}=\mathrm{v}_{2}{ }^{2} \quad \mathrm{r}_{2}=\mathrm{r} / 2$

$\mathrm{w}_{2}^{2}=\frac{\mathrm{v}_{2}^{2}}{\mathrm{r}_{2}{ }^{2}}=\mathrm{G} \frac{\mathrm{m}}{\mathrm{r}^{2} \mathrm{r}_{2}}=\mathrm{G} \frac{2 \mathrm{~m}}{\mathrm{r}^{3}}$

$\mathrm{K}_{2}=\frac{1}{2} \mathrm{mv}_{2}^{2}$

$\mathrm{K}_{2}=\frac{1}{2} \mathrm{mv}_{2}^{2}=\frac{1}{2} \mathrm{mG} \frac{\mathrm{m}}{2 \mathrm{r}}=\mathrm{G} \frac{\mathrm{m}^{2}}{4 \mathrm{r}}$

It is possible to obtain the same result, if we replace $m_{1}=m_{2}$ in the formula:

$$
\begin{aligned}
& \mathbf{K}_{1}=\frac{1}{2} G \frac{\mathbf{m}_{1} \mathbf{m}_{2}}{\mathbf{r}} \frac{\mathbf{m}_{2}}{\left(\mathbf{m}_{1}+\mathbf{m}_{2}\right)} \quad \mathrm{K}_{1}=\mathrm{G} \frac{\mathrm{m}^{2}}{4 \mathrm{r}} \quad \mathrm{m}_{1}=\mathrm{m}_{2}=\mathrm{m} \\
& \mathbf{K}_{2}=\frac{1}{2} \mathbf{G} \frac{\mathbf{m}_{1} \mathbf{m}_{2}}{\mathbf{r}} \frac{\mathbf{m}_{1}}{\left(\mathbf{m}_{1}+\mathbf{m}_{2}\right)} \quad \mathrm{K}_{2}=\mathrm{G} \frac{\mathrm{m}^{2}}{4 \mathrm{r}} \\
& \mathrm{K}_{\mathrm{T}}=\mathrm{K}_{1}+\mathrm{K}_{2}=2 \mathrm{G} \frac{\mathrm{m}^{2}}{4 \mathrm{r}} \\
& \mathrm{K}_{\mathrm{T}}=\mathrm{G} \frac{\mathrm{m}^{2}}{2 \mathrm{r}} \quad \mathrm{U}=-\mathrm{G} \frac{\mathrm{m}^{2}}{\mathrm{r}}
\end{aligned}
$$

Each mass $m$ has equal angular velocity, equal angular frequency, equal energy emission, equal linear velocity and equal kinetic energy. Thus, the total mechanical energy of the system of the binary stars is:

$E=K_{T}+U=G \frac{m^{2}}{2 r}-G \frac{m^{2}}{r}$

$E=-G \frac{m^{2}}{2 r}$

It is possible to obtain the total energy of the system from the formula:

$\mathrm{K}_{\mathrm{T}}=\mathrm{K}_{1}+\mathrm{K}_{2}=\frac{1}{2} \mathrm{G} \frac{\mathrm{m}_{1} \mathrm{~m}_{2}}{\mathrm{r}} \frac{\mathrm{m}_{2}}{\left(\mathrm{~m}_{1}+\mathrm{m}_{2}\right)}+\frac{1}{2} \mathrm{G} \frac{\mathrm{m}_{1} \mathrm{~m}_{2}}{\mathrm{r}} \frac{\mathrm{m}_{1}}{\left(\mathrm{~m}_{1}+\mathrm{m}_{2}\right)}$

$\mathrm{U}=-\mathrm{G} \frac{\mathrm{m}_{1} \mathrm{~m}_{2}}{\mathrm{r}} \quad$ For $\mathrm{m}_{1}=\mathrm{m}_{2}=\mathrm{m}$ and $\mathrm{r}_{1}=\mathrm{r}_{2}=\mathrm{r} / 2 \quad \mathrm{r}=\mathrm{r}_{1}+\mathrm{r}_{2}$

$E=K_{T}+U=2 G \frac{m^{2}}{4 r}-G \frac{m^{2}}{r}$ 
$E=-G \frac{m^{2}}{2 r}$

The mass of each star is as follows:

$\mathbf{m}_{1}=\mathbf{m}_{\mathbf{0} 1} \sqrt{\left(\mathbf{1}-\frac{\mathbf{v}_{\mathbf{1}}^{2}}{\mathbf{c}^{2}}\right)} \quad \mathbf{G} \frac{\mathbf{m}_{\mathbf{0}}}{2 \mathbf{r}}=\mathbf{v}_{\mathbf{1}}{ }^{2} \quad \mathrm{~m}_{\mathrm{o} 1}=\mathrm{m}_{\mathrm{o} 2}=\mathrm{m}_{\mathrm{o}}$

$\mathbf{m}_{2}=\mathbf{m}_{02} \sqrt{\left(1-\frac{\mathrm{v}_{2}^{2}}{\mathbf{c}^{2}}\right)} \quad \mathrm{G}_{\frac{\mathbf{m}_{0}}{2 \mathrm{r}}}=\mathbf{v}_{2}^{2}$

Then, each star has equal lost mass given by $\Delta \mathrm{m}=\mathrm{m}_{\mathrm{c}} \mathrm{c}^{2}-\mathrm{mc}^{2}$. Also, each star has equal gravitational energy emission [33]. It is possible to obtain the total energy at the classic approach:

$\mathrm{m}_{1}=\mathrm{m}_{\mathrm{o} 1}\left(1-\frac{\mathrm{v}_{1}^{2}}{2 \mathrm{c}^{2}}\right) \quad \mathrm{E}_{1}=\mathrm{m}_{1} \mathrm{c}^{2}-\mathrm{m}_{\mathrm{o} 1} \mathrm{c}^{2}=-\frac{\mathrm{v}_{1}^{2}}{2} \mathrm{~m}_{\mathrm{o} 1}$

$\mathrm{m}_{2}=\mathrm{m}_{\mathrm{o} 2}\left(1-\frac{\mathrm{v}_{2}{ }^{2}}{2 \mathrm{c}^{2}}\right) \quad \mathrm{E}_{2}=\mathrm{m}_{2} \mathrm{c}^{2}-\mathrm{m}_{\mathrm{o} 2} \mathrm{c}^{2}=-\frac{v_{2}^{2}}{2} m_{o 2}$

$\mathrm{E}=\mathrm{E}_{1}+\mathrm{E}_{2}=-\mathrm{G} \frac{\mathrm{m}_{\mathrm{o}}}{2 \mathrm{r}} \frac{\mathrm{m}_{\mathrm{o}}}{2}-\mathrm{G} \frac{\mathrm{m}_{\mathrm{o}}}{2 \mathrm{r}} \frac{\mathrm{m}_{\mathrm{o}}}{2} \quad \mathrm{~m}_{\mathrm{o} 1}=\mathrm{m}_{\mathrm{o} 2}=\mathrm{m}_{\mathrm{o}}$

$E=-G \frac{m_{o}^{2}}{2 r}$ as the classic result

\section{Conclusions}

This research presents the monitoring and analysis of the data taking of the Pierre Auger Observatory (PAO) and analysis of the parameters of the photomultipliers of the Surface Detector. The results of this analysis show that there are PMTs with bad behaviours, mainly with high fluctuations of the VEM Peak and/or the Dynode/Anode Ratio which are called High Changes PMT Gain. At these PMTs, it was observed large values only for the RMS of the VEM Peak and with the RMS of the Dynode/Anode Ratio approximately less than 0.5 . This bad behaviour also is an effect dependent on the temperature and mainly there is dependence with the higher temperatures. In order to select those PMTs a cut of RMS (VEM Peak) $>1$ ADC count and RMS (Dynode/Anode Ratio) $<0.5$ ADC counts should be used for the alarm creation.

At the end, the purpose of the monitoring data is to find problems with the data taking as soon as possible and in this form to do the respective alarm for them. Shifter actions have to be performed if the alarm is triggered. For the alarm creation procedure, firstly the analysis in order to find the threshold values for the parameters of the alarms should be done. The Free Disk alarm also was presented and this alarm is already installed in Auger Online Monitoring. This alarm is triggered when the disk of one local station has low capacity (less than 600000 Bytes). Finally, it is important to implement new alarms as soon as the experts report new future problem in the detectors.

Besides, it is researched about the measurement of the lifetime of muons and pions theoretically, experimentally and with the application of Statistics for the uncertainties of the results. The mean lifetime of muons and pions agree very well with the values cited in the literature. In particular for muons, the results are like they were expected. For example, the mean lifetime for muons in carbon obtained (material which the scintillator is 
basically composed) is $2.06 \mu \mathrm{s}$ and the value of the literature is $2.2 \mu \mathrm{s}$. For other hand, for pions the mean lifetime of $22.32 \mathrm{~ns}$ is a little closer of the experimental value which we were expected from the literature: $26 \mathrm{~ns}$. Therefore, the results have high accuracy and in accordance with the values of the literature.

At this article, it was demonstrated the discovered formula for the deflection angle of high energy cosmic rays in the propagation through the galactic and extragalactic magnetic fields. For other hand, it is possible to observe from the results of the deflection angles that they are very small for the considered cases. Thus, Ultra High Energy Cosmic Rays (UHECR) arrival directions should point back to their sources in the sky. An experiment able to provide data with high quality and statistical significance in the upper end of the cosmic ray spectrum, good angular resolution and the uniform exposure over the whole sky is necessary. The Pierre Auger Observatory was built with these objectives in mind [16],[19],[21], [22], [26], [27].

In addition, it was also demonstrated the GZK cutoff. For the analysis of the GZK cutoff, it is possible to conclude that:

- A nucleon at the Earth, which traveled a distance more than $100 \mathrm{Mpc}$, will have always an energy less than $10^{20} \mathrm{eV}$ independently of the initial energy.

- Particles with observed energies above $10^{20} \mathrm{eV}$ are limited to distances of approximately less than 100 Mpc from the Earth and also reduce their energy at about $10^{19} \mathrm{eV}$.

Nevertheless, the AGASA experiment showed a significant number of events with energies beyond the GZK cutoff. For this reason, a giant air shower array called the Pierre Auger Observatory is built to study these very rare high energy cosmic rays.

For other hand, Maxwell's theory shows that electromagnetic waves are radiated whenever charges accelerate as for example for the electrón. Then, this electromagnetic radiation (photons) produces a decrease in the electrón mass which is given by the formula of the Variant Mass for an Accelerated Charged Particle demonstrated by myself at this research [34] and at the research of the Variant Mass of the electron at the Atom [36]. Thus, this is true for any type of radiation emitted: electromagnetic or gravitational energy which produce a decrease in the mass of the body. Thus, other objective of this article is to demonstrate the discovered formula which describes exactly the variant mass of a particle which emits gravitational energy. An example of the effect of this Gravitational energy emission is the light deflection for the light passing close the Sun (gravitational redshift frequency) and the Perihelion Precession of Mercury [33].

The mass of the particle after the emission of the gravitational energy in a gravitational field (particle orbiting a Planet or Sun) is:

$\mathrm{m}=\mathrm{m}_{\mathrm{o}} \sqrt{\left(1-\frac{\mathrm{v}^{2}}{\mathrm{c}^{2}}\right)}$ mass for a particle in a gravitational field

$\left.\Delta \mathrm{m}=\mathrm{m}_{\mathrm{o}}\left(1-\sqrt{\left(1-\frac{\mathrm{GM}}{\mathrm{rc}^{2}}\right.}\right)\right) \quad \Delta \mathrm{m}=\mathrm{m}_{\mathrm{o}}-\mathrm{m}:$ total lost mass of the particle 
It is in agreement with the total mass decreasing of the particle in a gravitational field for the classic approach:

$\Delta \mathrm{m}=\mathrm{m}_{\mathrm{o}} \mathrm{c}^{2}-\mathrm{mc}^{2}=\left(\frac{\mathrm{GMm}_{\mathrm{o}}}{2 \mathrm{R}}\right) \quad \mathrm{GM} / \mathrm{r}<<\mathrm{c}^{2}$

The total energy for a particle in a gravitational field is as follows:

$\mathrm{E}=m c^{2}-m_{o} c^{2}$

$E=m_{o} c^{2}\left(\sqrt{\left(1-\frac{G M}{r c^{2}}\right.}-1\right)$ energy for a particle in a gravitational field

$\mathrm{E}=-\frac{G M m_{0}}{2 r} \quad$ (total energy at the classic approach) $\mathrm{GM} / \mathrm{r}<<\mathrm{c}^{2}$

The total mass energy lost is given by the formula demonstrated and it is equal in absolute value to the total bond energy of the system (particle orbiting a Planet or Sun) which is emitted as gravitational energy when the particle goes from one orbit to another orbit with less radius. The emission of the total gravitational energy E produces a lost mass of the particle $\mathrm{m}=\mathrm{E} / \mathrm{c}^{2}$.

If $\mathrm{R}_{\mathrm{f}}<\mathrm{R}_{\mathrm{i}} \Delta E=E_{f}-E_{i}=\left(-\frac{G M m}{2 R_{f}}\right)-\left(-\frac{G M m}{2 R_{i}}\right)$ is negative, it is given out energy which means that the particle losses mass, the particle increases the kinetic energy and the velocity but decreases the gravitational potential energy (more negative). Part of the losses energy (gravitational energy emission) is given out by decreasing the potential energy and part by increasing the kinetic energy [33].

If $\mathrm{R}_{\mathrm{f}}>\mathrm{R}_{\mathrm{i}} \Delta \mathrm{E}$ is positive, which means that additional energy is given to the particle or additional work is done on the system, the particle decreases the kinetic energy and the velocity but increases the potential energy (less negative). Part of the work or additional energy is used to increase the potential energy and part to diminish the kinetic energy [33].

The same process occurs in the atom for the electron when jump from one orbit to other. The particle decreases its radio and the velocity $\mathrm{v}$ increases with emission of electromagnetic energy (photon) at the jump of the electron from one orbit to another with less radius. Besides, the electron losses mass as result of this emission. It is established at the article The Fundament of the Mass: The Variant Mass of Electron at Atom where it is demonstrated the formula discovered by myself for the Variant Mass of the Electron [36]. It is possible to appreciate the symmetry of the physics and the nature by comparing what happen at the atom (microscopic scale) and at the planetary system or space (macroscopic scale). Also, the mass for the particle in a gravitational field which uses a Newton Approach agrees with the formula for the mass from General Relativity at distances very far from the massive object (Sun for example) and also at distances very near to the massive object with high accuracy [33]. Then, both models: the Newton Approach with a gravitational field interaction and gravitation force and the Relativity Theory with a curvature of the space time are in agreement at the results for the mass of the particle in a gravitational field with high accuracy.

Besides, it is possible to have the next thought experiment: If a mass $M_{o}$ is suspended from a spring balance in the $\mathrm{K}$ ' accelerated system with acceleration $\mathrm{g}$, the balance will indicate the apparent weight $\mathrm{M}_{\mathrm{o}} \mathrm{g}$ due to the inertia of 
$M_{o}$ (the balance transmits the acceleration to the mass $M_{o}$ ) [1]. If the amount of energy $E$ is transferred to $M_{o}$, the spring balance, by the law of the inertia of the energy (equivalence of mass and energy $\mathrm{E} / \mathrm{c}^{2}=\mathrm{m}$ ), will indicate $M_{o} g+\frac{E}{c^{2}} g$. Exactly, this should happen when the experiment is repeated in the gravitational system $\mathrm{K}$ where the balance transmits the gravity acceleration of the Earth to the mass $M_{0}$. It happens also at the light deflection when passing close the Sun. The light does not have mass but light has energy and as consequence, it experiments the interaction with the gravitational field and the light deflection (gravitational redshift frequency) [33]. Then, the light passing near the Sun for example experiments the gravitational redshift when the light is observed a the Earth: less frequency measured, deviation to the red, more wavelength and real lost energy [33]. Besides, other result of the gravitational interaction is the Perihelion Precession of Mercury. The perihelion or the ellipse of Mercury has a small rotation and changes a small angle $\theta$ after every turn around the Sun (about 43 arcseconds by century). The light deflection and the Perihelion Precession of Mercury are demonstrated at the article: The Fundament of the Mass and Effects of the Gravitation on a Particle and Light in the Mass, Time, Distance, Velocity, Frequency, Wavelength: Variant Mass for a Particle which emits Gravitational Energy for a Particle Orbiting a large Planet or Sun and for a Binary Star and Variant frequency for the Light Passing Close a Gravitational Field from a Massive Object (Sun) [33].

For a Binary Star, the formula for the decrease mass is as follows:

$$
\begin{array}{ll}
m_{1}=m_{o 1} \sqrt{\left(1-\frac{v_{1}^{2}}{c^{2}}\right)} \quad G \frac{m_{o 2}{ }^{2}}{r\left(m_{o 1}+m_{o 2}\right)}=v_{1}{ }^{2} \\
m_{2}=m_{o 2} \sqrt{\left(1-\frac{v_{2}^{2}}{c^{2}}\right)} & G \frac{m_{o 1}{ }^{2}}{r\left(m_{o 1}+m_{o 2)}\right.}=v_{2}{ }^{2}
\end{array}
$$

Then, each star has different lost mass given by $\Delta m=m_{o} c^{2}-m c^{2}$. Also, each star has different gravitational energy emission corresponding to the lost mass of the respective star. Besides, each star with mass $\mathrm{m}$ has equal angular velocity $w$, equal angular frequency $\mathrm{f}(\mathrm{w}=2 \pi \mathrm{f})$ and different kinetic energy with different linear velocities $\mathrm{v}$.

The total energy for each particle is as follows:

$$
\begin{array}{ll}
\mathrm{E}_{1}=\mathrm{m}_{1} \mathrm{c}^{2}-\mathrm{m}_{01} \mathrm{c}^{2} m_{1}=m_{o 1} \sqrt{\left(1-\frac{v_{1}{ }^{2}}{c^{2}}\right)} & G \frac{m_{o 2}{ }^{2}}{r\left(m_{o 1}+m_{o 2}\right)}=v_{1}{ }^{2} \\
\mathrm{E}_{2}=\mathrm{m}_{2} \mathrm{c}^{2}-\mathrm{m}_{02} \mathrm{c}^{2} m_{2}=m_{o 2} \sqrt{\left(1-\frac{v_{2}{ }^{2}}{c^{2}}\right)} & G \frac{m_{o 1}{ }^{2}}{r\left(m_{o 1}+m_{o 2)}\right.}=v_{2}{ }^{2}
\end{array}
$$

Therefore, it is possible to obtain for the total energy at the classic approach:

$$
\begin{array}{ll}
m_{1}=m_{o 1}\left(1-\frac{v_{1}{ }^{2}}{2 c^{2}}\right) & \mathrm{E}_{1}=\mathrm{m}_{1} \mathrm{c}^{2}-\mathrm{m}_{\mathrm{o} 1} \mathrm{c}^{2}=-\frac{v_{1}{ }^{2}}{2} m_{o 1} \\
m_{2}=m_{o 2}\left(1-\frac{v_{2}{ }^{2}}{2 c^{2}}\right) & \mathrm{E}_{2}=\mathrm{m}_{2} \mathrm{c}^{2}-\mathrm{m}_{\mathrm{o} 2} \mathrm{c}^{2}=-\frac{v_{2}{ }^{2}}{2} m_{o 2}
\end{array}
$$

The total energy of the system is:

$E=-G \frac{m_{01} m_{2}}{2 r}$ as the classic result 
If both stars have equal mass: $\mathrm{m}_{\mathrm{o} 1}=\mathrm{m}_{\mathrm{o} 2}$, then each star with mass $\mathrm{m}$ orbits the same circle of radius $\mathrm{r}_{1}=\mathrm{r}_{2}$. The mass of each star is as follows:

$$
\begin{aligned}
& \mathrm{m}_{1}=\mathrm{m}_{\mathrm{o} 1} \sqrt{\left(1-\frac{\mathrm{v}_{1}^{2}}{\mathrm{c}^{2}}\right)} \quad \mathrm{G} \frac{\mathrm{m}_{\mathrm{o}}}{2 \mathrm{r}}=\mathrm{v}_{1}{ }^{2} \quad \mathrm{~m}_{\mathrm{o} 1}=\mathrm{m}_{\mathrm{o} 2}=\mathrm{m}_{\mathrm{o}} \\
& \mathrm{m}_{2}=\mathrm{m}_{\mathrm{o} 2} \sqrt{\left(1-\frac{\mathrm{v}_{2}^{2}}{\mathrm{c}^{2}}\right)} \quad \mathrm{G} \frac{\mathrm{m}_{\mathrm{o}}}{2 \mathrm{r}}=\mathrm{v}_{2}{ }^{2}
\end{aligned}
$$

Each mass $m$ has equal angular velocity, equal angular frequency, equal energy emission, equal linear velocity and equal kinetic energy. Then, each star has equal lost mass given by $\Delta \mathrm{m}=\mathrm{m}_{\mathrm{o}} \mathrm{c}^{2}-\mathrm{mc}^{2}$.

Also, each star has equal gravitational energy emission [33]. The total energy for each particle is as follows:

$$
\begin{aligned}
& \mathrm{E}_{1}=\mathrm{m}_{1} \mathrm{c}^{2}-\mathrm{m}_{\mathrm{o} 1} \mathrm{c}^{2} \quad m_{1}=m_{o 1} \sqrt{\left(1-\frac{v_{1}^{2}}{c^{2}}\right)} \quad \mathrm{G} \frac{\mathrm{m}_{\mathrm{o}}}{2 \mathrm{r}}=\mathrm{v}_{1}{ }^{2} \quad \mathrm{~m}_{\mathrm{o} 1}=\mathrm{m}_{\mathrm{o} 2}=\mathrm{m}_{\mathrm{o}} \\
& \mathrm{E}_{2}=\mathrm{m}_{2} \mathrm{c}^{2}-\mathrm{m}_{\mathrm{o} 2} \mathrm{c}^{2} \quad m_{2}=m_{o 2} \sqrt{\left(1-\frac{v_{2}^{2}}{c^{2}}\right)} \quad \mathrm{G} \frac{\mathrm{m}_{\mathrm{o}}}{2 \mathrm{r}}=\mathrm{v}_{1}{ }^{2}
\end{aligned}
$$

It is possible to obtain the total energy at the classic approach:

$$
\begin{aligned}
& \mathrm{m}_{1}=\mathrm{m}_{\mathrm{o} 1}\left(1-\frac{\mathrm{v}_{1}^{2}}{2 \mathrm{c}^{2}}\right) \quad \mathrm{E}_{1}=\mathrm{m}_{1} \mathrm{c}^{2}-\mathrm{m}_{\mathrm{o} 1} \mathrm{c}^{2}=-\frac{\mathrm{v}_{1}^{2}}{2} \mathrm{~m}_{\mathrm{o} 1} \\
& \mathrm{~m}_{2}=\mathrm{m}_{\mathrm{o} 2}\left(1-\frac{\mathrm{v}_{2}^{2}}{2 \mathrm{c}^{2}}\right) \quad \mathrm{E}_{2}=\mathrm{m}_{2} \mathrm{c}^{2}-\mathrm{m}_{\mathrm{o} 2} \mathrm{c}^{2}=-\frac{v_{2}{ }^{2}}{2} m_{o 2} \\
& \mathrm{E}=\mathrm{E}_{1}+\mathrm{E}_{2}=-\mathrm{G} \frac{\mathrm{m}_{\mathrm{o}}}{2 \mathrm{r}} \frac{\mathrm{m}_{\mathrm{o}}}{2}-\mathrm{G} \frac{\mathrm{m}_{\mathrm{o}}}{2 \mathrm{r}} \frac{\mathrm{m}_{\mathrm{o}}}{2} \quad \mathrm{~m}_{\mathrm{o} 1}=\mathrm{m}_{\mathrm{o} 2}=\mathrm{m}_{\mathrm{o}} \\
& \mathrm{E}=-\mathrm{G}^{\mathrm{m}} \frac{\mathrm{o}_{\mathrm{o}}^{2}}{2 \mathrm{r}} \text { as the classic result }
\end{aligned}
$$

The results of the mass formula are of great relevance for Gravitational Interactions.

The results are in accordance with the classic result for the emission of the total gravitational energy (bond total energy) for a particle orbiting a large Planet or Sun and for a Binary Star. It is in agreement with the experiment result and with the Theory of General Relativity [33].

\section{Declarations}

\section{Source of Funding}

This research did not receive any specific grant from funding agencies in the public, commercial, or not-for-profit sectors.

\section{Competing Interests Statement}

The author declares no competing financial, professional and personal interests.

\section{Ethical Approval}

Not Applicable. 
Consent to participate

The consent to participate in this research was sought for and approved by the subjects to be used.

\section{Consent for publication}

Author declares that he/she consented for the publication of this research work.

\section{Availability of data and material}

Author is willing to share data and material according to the relevant needs.

\section{References}

[1] Emilio Segré, Nuclei and Paricles, The Benjamin/Cummings Publishing Company, Inc. 1977.

[2] M. T. Dova (Pierre Auger Collaboration), Survey of the Pierre Auger Observatory, Proc. ICRC 2001.

[3] Donald Perkins, Particle Astrophysics, Oxford University Press, 2003.

[4] C. Grupen, Astroparticle Physics, Springer-Verlag Berlin Heidelberg, 2005.

[5] M. Lemoine, Physics and Astrophysics of Ultra-High Energy Cosmic Rays, Springer-Verlag Berlin Heidelberg 2001.

[6] R.W. Clay, A. G. K. Smith and J. L. Reid, Cosmic Ray Induced Noise in Gravitation Wave Detectors, Electronic Publications of the Astronomical Society of Australia, Vol. 14, Number 2.

[7] Pierre Auger in Collaboration with P. Ehrenfest, R. Maze, J. Daudin, Robley, A. Freon, Extensive Cosmic-Ray Showers, Rev. of Modern Physics, Vol.11, 288-291 (1939).

[8] Symmetry, volume02, issue01, February 05.

[9] Bluemer, J. (Pierre Auger Collaboration), Status and Perspectives of the Pierre Auger Observatory, Proc. The 28th International Cosmic Ray Conference.

[10] Iona Dutan, Laurentiu Caramete, Alex Curutiu, Iona C. Maris, Ona Tascau, Peter L. Biermann, The origin of Ultra high energy cosmic rays: A complete sample of predicted sources and a predicted sky distribution of events (UHECR-X), GAP Note 2007-109.

[11] Todor Stanev, Ultra-High-Energy Cosmic Rays and the Large-Scale Structure of the Galactic Magnetic Field, Astrophysical Journal, Report Number BRI-96-32, 1997.

[12] Oliver Deligny, Antoine Letessier-Selvon, Etienne Parizot, Magnetic horizons of UHECR sources and the GZK feature, Elsevier Science, 2007.

[13] Pierre Auger Collaboration, The Pierre Auger Observatory Design Report, Second Edition, 1997.

[14] Nishant Mehta, Helio Takai, Simulation and Detection of Extensive Air Showers, Brookhaven National Laboratory, 2003. 
[15] Ronald Cintra Shellard (Pierre Auger Collaboration), First Results from the Pierre Auger Observatory, Brazilian Journal of Physics, Vol. 36, No.4, 2006.

[16] M. Kleifges and H. Gemmeke (IEEE for the Auger Collaboration), UHE Cosmic Ray Detection with the Pierre Auger Observatory, IEEE Transactions on Nuclear Science, Vol. 51, Issue 3, Part 1, 2004.

[17] Enrique Zas, ZeV Air Showers: The View from Auger, Article from Physics Department, University of Santiago of Compostela.

[18] B. Genolini, L. Raux, C. de La Taille, J. Pouthas, V. Tocut, A Large Dynamic Range Integrated Front-End for Photomultiplier Tubes, Beaune, 2005, Poster I-8.

[19] D. Nitz (Pierre Auger Collaboration), The Front-End Electronics for the Pierre Auger Observatory Surface Array, IEEE Vol.51, No.3, 2004.

[20] X. Bertou, P. S. Allison, C. Bonifazi, P. Bauleo (Pierre Auger Collaboration), Calibration of the Surface Array of the Pierre Auger Observatory, GAP note: 06-012.

[21] A. tripathi, X. Bertou, A. Chou, A systematic calibration of Surface Detectors using Muon Data from the Engineering Array, GAP note 2002-046.

[22] M.A. L. de Oliveira, Pierre Auger Observatory: status report, Brazilian Journal of Physics, Vol.34, 4a, 2004.

[23] Xavier Bertou (Pierre Auger Collaboration), Calibration of the surface array of the Pierre Auger Observatory, 29th International Cosmic Ray Conference Pune, 2005.

[24] Johannes Bluemer (Pierre Auger Collaboration), Status and Perspectives of the Pierre Auger Observatory, Acta Physical Polonica B, No 6-7, Vol. 35, 2004.

[25] D. Allard, E. Armengaud, I. Allekotte, The trigger system of the Pierre Auger Surface Detector: operation, efficiency and stability, Proc. of 29th International Cosmic Ray Conference Pune, 2005.

[26] Cyril Lachaud (Pierre Auger Collaboration), Pierre Auger Observatory: Overview of the Acquisition Systems, LPCC/CDF, Paris, France.

[27] R. Meyhandan, J. Matthews, D. F. Nitz, Auger front-end ASIC simulations, Proc. ICRC 2001: 777, GAP note: 2001-039.

[28] Paul Lebrun, On the Fluctuating PMT gain, SD Monitoring Analysis, GAP Note-2007-042.

[29] W. Fulgione, About raining PMTs, GAP Note-2007-081.

[30] P. Frenkel, JM. Brunet, L. Guglielmi, G. Tristan, Evaluation of the Central Station Hardware, France, 1997, GAP note: $1997-041$.

[31] Auger Collaboration, Properties and performance of the prototype instrument for the Pierre Auger Observatory, Nuclear Instruments \& Methods in Physics Research A 523, 2004. 
[32] Giovanni Alcocer, Xavier Alcocer, Deflection angle of high energy cosmic rays in the propagation through the galactic and extragalactic magnetic fields and the GZK (Greisen-Zatsepin-Kuzmin) cutoff, Mediterranean Journal of Basic and Applied Sciences, (MJBAS), Volume 2, Issue 2, Pages 37-47, Abril-June 2018.

[33] Giovanni Alcocer, The Fundament of the Mass and Effects of the Gravitation on a Particle and Light in the Mass, Time, Distance, Velocity, Frequency, Wavelength: Variant Mass for a Particle which emits Gravitational Energy for a Particle Orbiting a large Planet or Sun and for a Binary Star and Variant Frequency for the Light Passing Close a Gravitational Field from a Massive Object (Sun), Mediterranean Journal of Basic and Applied Sciences (MJBAS), Volume 4, Issue 3, Pages 42-69, July-September 2020.

[34] Giovanni Alcocer,Variant Mass for an Accelerated Charged Particle, Asian Journal of Basic Science \& Research, Volume 2, Issue 3, Pages 43-59, July-September 2020.

[35] Giovanni Alcocer, Cosmic Rays and Measurement of the lifetime of muons and pions, Physics of the Cosmic Rays: Theory, Experiment and Statistics, Lambert Academic Publishing, 2015.

[36] Giovanni Alcocer, The Fundament of the Mass: The Variant Mass of Electron at Atom, Meditteranean Journal of Basic and Applied Sciences (MJBAS), Volume 4, Issue 4, Pages 18-113, October-December 2020. 